
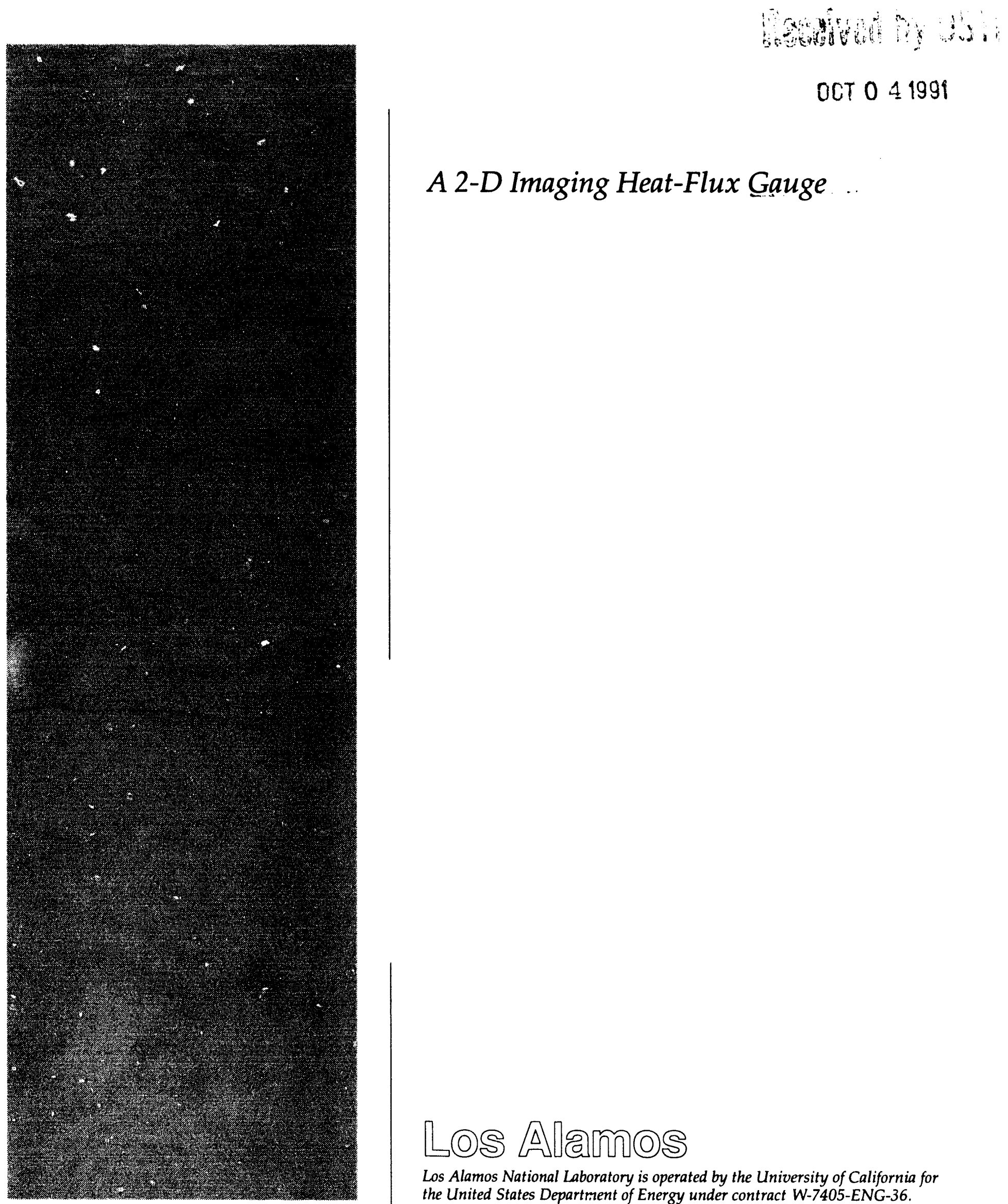

OCT 041999

\title{
A 2-D Imaging Heat-Flux Gauge
}

\section{Los Allamos}

Los Alamos National Laboratory is operated by the University of California for the United States Department of Energy under contract W-7405-ENG-36. 
Edited by Lilly Shelley, MEE-DO

This work was supported by Wright Laboratory, Turbine Engine Division, Components Branch

An Affirmative Action/Equal Opportunity Employer

This report was prepared as an account of work sponsored by an agency of the

United States Government. Neither The Regents of the University of California, the United States Government nor any agency thereof, nor any of their employees, makes any warranty, express or implied, or assumes any legal liability or responsibility for the accuracy, completeness, or usefulness of any information, apparatus, product, or process disclosed, or represents that its use would not infringe privately owned rights. Reference herein to any specific commercial product. process, or service by trade name, trademark, manufacturer, or otherwise, does not necessarily constitute or imply its endorsement, recommendation, or favoring by The Regents of the University of California, the United States Government or any agency thereof. The views and opinions of authors expressed herein do not necessarily state or reflect those of The Regents of the University of California, the United States Government or any agency thereof. 


\title{
A 2-D Imaging Heat-Flux Gauge
}

\author{
B. W. Noel \\ D. L. Beshears* \\ H. M. Borella* \\ W. K. Sartory* \\ K. W. Tobin* \\ W. D. Turleyt \\ R. K. Williams*
}

*Oak Ridge National Laboratory, P.O. Box 2003, Oak Ridge, TN 37831.

*"Former consultant, Los Alamos National Laboratory; now deceased.

+EGEG Energy Measurements, Inc., Santa Barbara Operations, Goleta, CA 93116.

\section{MASTER}

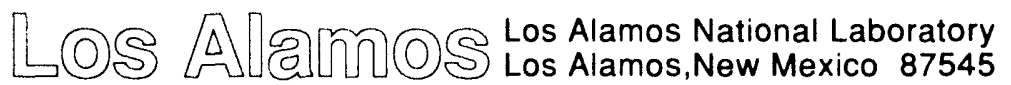




\title{
A 2-D IMAGING HEAT-FLUX GAUGE
}

by

\author{
B. W. Noel, ${ }^{*}$ D. L. Beshears, ${ }^{* *}$ H. M. Borella, ${ }^{\dagger}$ W. K. Sartory, ${ }^{* *}$ K. W. Tobin, ${ }^{* *}$ \\ W. D. Turley $\ddagger$ and R. K. Williams**
}

\begin{abstract}
This report describes a new leadless two-dimensional imaging optical heat-flux gauge. The gauge is made by depositing arrays of thermographic-phosphor (TP) spots onto the faces of a polymethylpentene insulator. In the first section of the report, we describe several gauge configurations and their prototype realizations. A satisfactory configuration is an array of right triangles on each face that overlay to form squares when the gauge is viewed normal to the surface. The next section of the report treats the thermal conductivity of TPs. We set up an experiment using a comparative longitudinal heat-flow apparatus to measure the previously unknown thermal conductivity of these materials. The thermal conductivity of one TP, $\mathrm{Y}_{2} \mathrm{O}_{3}: \mathrm{Eu}$, is $0.0137 \mathrm{~W} / \mathrm{cm} \cdot \mathrm{K}$ over the temperature range from about 300 to $360 \mathrm{~K}$. The theories underlying the time response of TP gauges and the imaging characteristics are discussed in the next section. Then we discuss several laboratory experiments to (1) demonstrate that the TP heat-flux gauge can be used in imaging applications; (2) obtain a quantum yield that enumerates what typical optical output signal amplitudes can be obtained from TP heat-flux gauges; and (3) determine whether LANL-designed intensified video cameras have sufficient sensitivity to acquire images from the heat-flux gauges. We obtained positive results from all the measurements. Throughout the text, we note limitations, areas where improvements are needed, and where further research is necessary.
\end{abstract}

\section{INTRODUCTION}

We recently demonstrated a new leadless optical heat-flux gauge. ${ }^{1}$ The gauge is made in a "sandwich" configuration, with two layers of thermographic phosphors (TPs) applied to the faces of a sheet of polymethylpentene (PMP) insulator. The gauge is attached to a surface upon which heat flux impinges. The heat flux is calculated by measuring the temperature of the two TPs and substituting the values obtained into the standard formula,

$$
q=k \Delta T / d
$$

where $q$ is the heat flux, $k$ is the thermal conductivity of the insulator, $\Delta T$ is the temperature differential across the gauge, and $d$ is the thickness of the insulator.

* Los Alamos National Laboratory

** Oak Ridge National Laboratory

$\dagger$ Formerly consultant, Los Alamos National Laboratory; now deceased

$\ddagger$ EG\&G Energy Measurements, Santa Barbara Operations 
Because this new gauge has no leads. the gauge can, in principle, cover an entire surface and measure the two-dimensional (2-D) spatial distribution of the heat flux incident on that surface.

This report summarizes the progress that we have made toward developing a 2-D imaging heatflux gauge. In the following sections, we discuss 2-D heat-flux gauge configurations, laboratoryprototype realizations of those gauges, and environmental testing of the prototypes; thermalconductivity measurements on the TP materials; some theoretical aspects of the heat flow in the gauges and of imaging them; an in-laboratory demonstration of a 2-D gauge; a description of the progress that has been made toward the false-color mapping of the spatial temperature distribution: and the initial design of an experiment that will use the 2-D heat-flux gauge in a full-scale demonstration.

For the prototype single-point heat-flux gauge, we assumed that the gauge would be required to operate in the steady-state heat-flux mode with a subject-surface temperature of $70^{\circ} \mathrm{F}$, a temperature differential of about $30^{\circ} \mathrm{F}$, and an impinging-gas temperature of $300^{\circ} \mathrm{F}$ to $500^{\circ} \mathrm{F}$. This set of conditions has not changed for the ultimate gauge, but a new set has evolved for the full-scale 2-D demonstration experiment that is described in $\S$ VI. In this experiment, the component side of the heat-flux gauge is heated to a maximum of $125^{\circ} \mathrm{F}$, and the impinging-gas temperature is near ambient.

\section{2-D GAUGE CONFIGURATIONS}

Like the single-point heat-flux gauge prototype, the 2-D gauge is of a sandwich construction, consisting of TP layers deposited on the faces of the insulator. We fabricated sample gauges by several different methods and in several geometries, each of which reflects a particular design philosophy. The main difference between the 2-D gauge and the single-point version is that each gauge, when viewed normal to its surface (as in Fig. 1), acts as an individual pixel in a gridded array of gauges. The size of the pixels is determined by the desired spatial resolution. The grid pattern provides an easy-to-identify location of each gauge/pixel relative to the surface to which the gauge is attached. It also leaves an adequate area of PMP available for bonding to the substrate.

In the design shown in Fig. 1, gauge elements were deposited by air brushing TP onto one face of the PMP substrate through a stencil of half-circles.

The other TP was applied to the opposite face of the PMP using the same stencil. The two semicircular grids were matched to form a single circular spot $\approx 1 \mathrm{~mm}$ in diameter for each pixel. This configuration lets light from the ultraviolet (UV) excitation source pass into, and the resulting fluorescence exit from. the top- and bottom-surface TPs individually, without excessive attenuation and without ambiguity about which fluorescence comes from which face of the gauge.

An apparent advantage of this gauge design is that the fraction of the gauge-surface area not covered with phosphor is relatively large, which leaves ample surface area available to glue the gauge array to the test surface. In practice. this feature turned out to be unimportant: other gauge designs also have enough surface not covered with TP to assure adequate adhesion.

The theory developed in $§ I V . A$ assumes that the TP particles are buried under the surface and in intimate thermal contact with the bonding agent and the plastic, with no voids or other flaws that would introduce an additional unknown thermal resistance. The bonding method, TP, and plastic must be laid out and fitted together in such a way that this assumption is not violated. We take great pains to fabricate heat-flux gauges accordingly. 


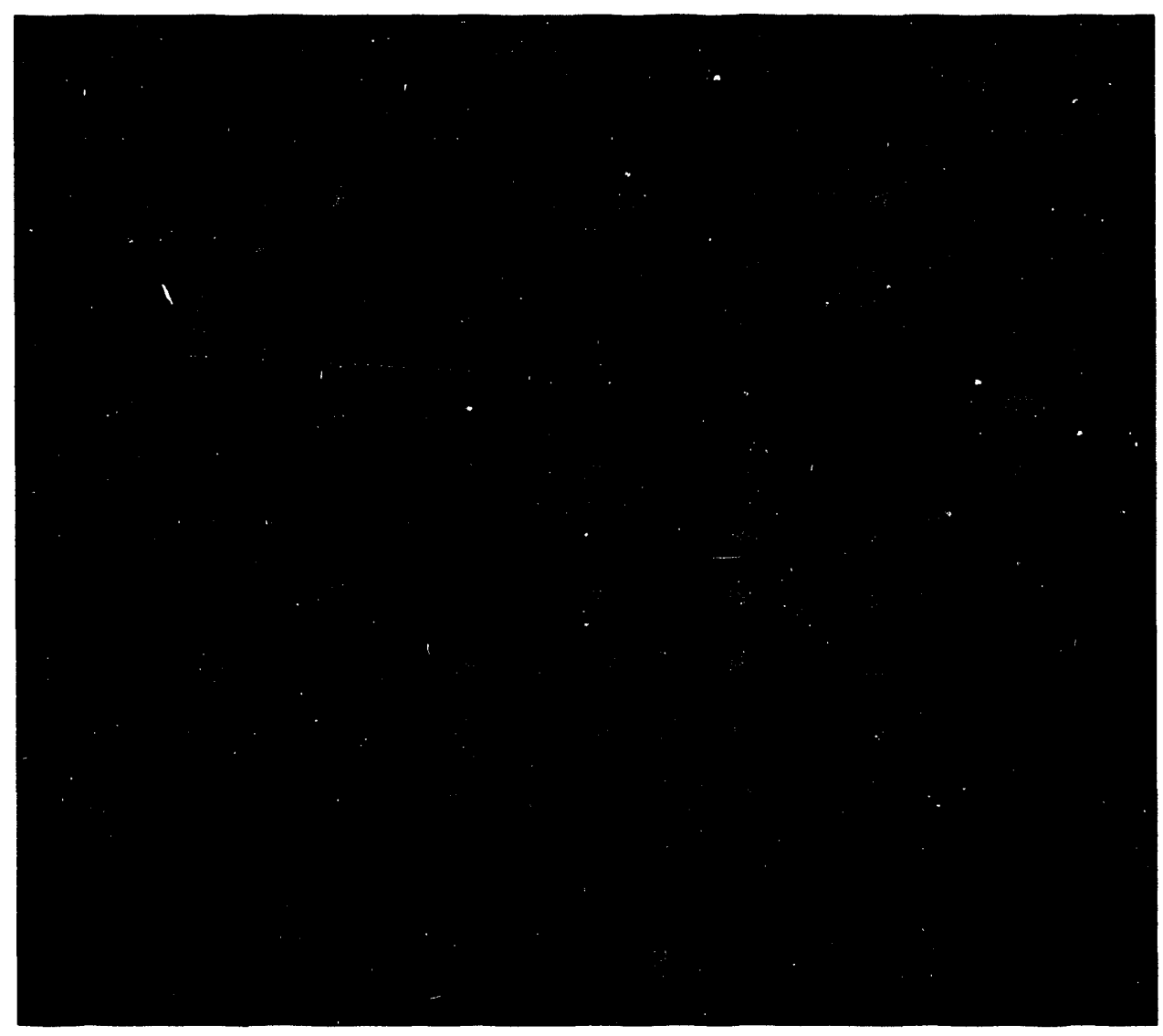

Fig. 1. 2-D heat-flux gauge, semicircular pattern. The $\mathrm{Gd}_{2} \mathrm{O}_{2} \mathrm{~S}$ :Tb shows up as green and the $\mathrm{La}_{2} \mathrm{O}_{2} \mathrm{~S}: \mathrm{Eu}$ as yellow.

A superior design is shown in Fig. 2. This gauge is laid out on each face with right triangles that match to form square pixels. The gauge is illuminated by a nercury-arc UV lamp. The $\mathrm{Gd}_{2} \mathrm{O}_{2} \mathrm{~S}: \mathrm{Tb}$ TP on the top of the gauge (in the terminology used here, the top is closest to the viewer) shows $u_{1}$, green and the $\mathrm{La}_{2} \mathrm{O}_{2} \mathrm{~S}: \mathrm{Eu}$ on the bottom is yellow. The ratio of active gauge area to total surface area can be maximized with this gauge design. The inactive area needs only to be large enough to permit effective attachment to the surface under test.

The TP is attached to this heat-flux gauge array, as before, by air brushing. The air-brushing working fluid is a slurry of acetone and TP, combined with a small percentage of organic binder. The technique was described in Reference 1. Although rf sputtering is promising as a TP deposition method, we have yet to use it successfully to make a heat-flux gauge. Sputtering melts the plastic insulator material. It also decomposes oxysulfide TPs when they are deposited. Annealing to recrystallize them requires the use of hot $\mathrm{H}_{2} \mathrm{~S}$, which will also melt the plastic.

We learned recently of a new form of Teflou that is available in clear, UV-transparent sheets in a variety of thicknesses. The sheets can be cemented or heat-bonded. We need to determine whether the thermal parameters have suitable values for use in a heat-flux gauge.

The air-brushed TP is vulnerable to abrasion and smearing during handling and experimentation. We have been studying various protective overcoating methods. One material, parylene, works well 


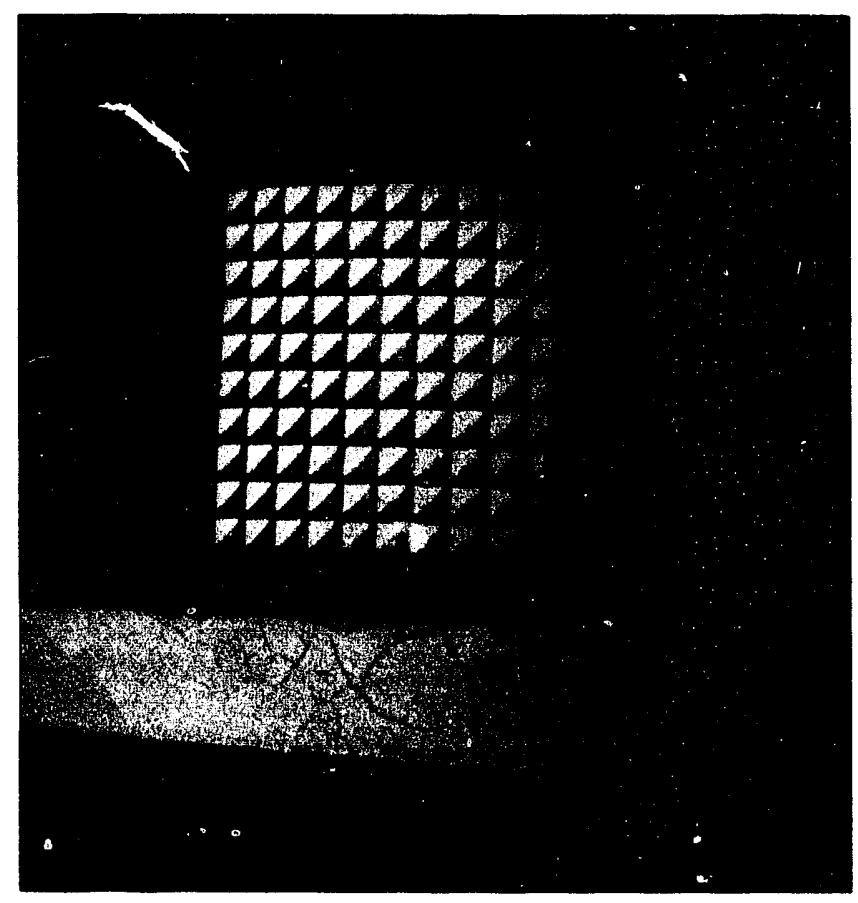

Fig. 2. Close-up view of triangular-pattern heat-flux gauge excited by a UV lamp.

as a coating, but it fluoresces strongly at the TP emission wavelength and is therefore not suitable. The search continues.

Bonding of the heat-flux gauge to metallic substrates with gap-fill dental cement, a cyanoacrylate, provided the best bond of the adhesives surveyed. The gauge-to-metal bond exceeds the tensile strength of $50-\mu \mathrm{m}$-thick PMP film.

The TPs selected for the previous versions of the gauge are europium-activated lanthanum oxysulfide, $\mathrm{La}_{2} \mathrm{O}_{2} \mathrm{~S}$ :Eu, and europium-activated gadolinium oxysulfide, $\mathrm{Gd}_{2} \mathrm{O}_{2} \mathrm{~S}$ :Tb. Reasons for the particular choices are discussed in Reference 1. The newest version of the heat-flux gauge uses $\mathrm{Gd}_{2} \mathrm{O}_{2} \mathrm{~S}$ : Tb on both the front and back surfaces. The spatial separation of the half pixels in the plane of the gauge permits separation of the signals from the front and back surfaces. This design has the added advantage of requiring only two band-pass filters instead of four, and therefore requires only two images (or video fields) per measurement.

We determined the effect of heat cycling on $\mathrm{La}_{2} \mathrm{O}_{2} \mathrm{~S}$ :Eu and $\mathrm{Gd}_{2} \mathrm{O}_{2} \mathrm{~S}$ :Tb powders by cycling them repeatedly in ovens from ambient to $150^{\circ} \mathrm{F}$. We monitored the emission of the TPs during the cycling tests. Optical fibers delivered UV excitation (from a mercury-arc lamp filtered to select the UV line at $254 \mathrm{~nm}$ ) and collected the visible emission from the TPs. The emission was recorded and processed with a diode array and an optical multichannel analyzer. Within the uncertainty imposed by systematic error, no change was observed in the TPs with temperature cycling.

\section{THERMAL CONDUCTIVITY}

An important part of the effort was directed at measuring the thermal conductivity of TPs in their as-deposited form. A thorough literature search disclosed no data on the thermal conductivity for either $\mathrm{La}_{2} \mathrm{O}_{2} \mathrm{~S}: \mathrm{Eu}$ or $\mathrm{Gd}_{2} \mathrm{O}_{2} \mathrm{~S}$ :Tb. Data do exist for similar materials, including $\mathrm{La}_{2} \mathrm{O}_{3}$ and $\mathrm{Gd}_{2} \mathrm{O}_{3}$. It turns out, however, that the thermal conductivity strongly depends on the percentage 
of the dopant. Thus data for similar materials are unusable; data for the actual materials are necessary for accurately calibrating the gauge (because the thermal conductivity appears in the heat-flux formula) if the gauge is to be calibrated directly. Although it is possible to calibrate gauges secondarily by comparison with a reference gauge, the TP heat-flux gauge can only be as accurate as the gauge used for a reference. When the gauge is calibrated secondarily, one can infer the thermal conductivity as long as the insulator thickness is known accurately and the effects of the nonzero TP thickness are properly accounted for.

For our initial work on the prototype heat-flux gauge, we estimated the thermal conductivity by interpolation or extrapolation from data on somewhat similar materials. We then explored the possibility of obtaining accurately measured values of thermal conductivity for the proposed gauge materials. This proved more difficult than it first appeared. A thorough literature search of the many possible configurations of an apparatus for measuring the thermal conductivity turned up several candidates. Careful analysis showed that all of these candidates had flaws that made them less than satisfactory. After a frustrating search, we finally discovered an established calibration facility at Oak Ridge National Laboratory that promised accurate results on our materials. An impressive facility, it nevertheless required careful experiment design, and funds were adequate for only one experimental run. This run was made using europium-activated yttrium oxide (our most thoroughly characterized TP), $\mathrm{Y}_{2} \mathrm{O}_{3}:$ Eu. The method of making pressed test samples of the TPs has been worked out and tested. If more funds become available, the thermal conductivity of all candidate TPs can now be measured.

\section{A. Procedure}

The thermal conductivity was measured in a comparative longitudinal heat-flow apparatus. ${ }^{2}$ Figure 3 is a photograph of the test apparatus. The instrument was designed to measure the thermal conductivity of small specimens over a modest temperature range without having to place thermocouples directly on the sample. The method was originally intended for materials that could only be instrumented with extreme difficulty because of specimen properties, size, or radiation hazards. This method is especially useful for our TP materials because they are necessarily small and have very low thermal conductivity.

When following the standard testing procedure, the results have an experimental uncertainty of about $\pm 3 \%$. This was verified previously by making measurements on NBS (now NIST) Standard Reference Material. Measurements of the thermal conductivity of Pyroceram 9606 (an insulator) deviated from NBS (NIST) measurements by less than $3 \%$ over the temperature range investigated. ${ }^{2}$

In the apparatus shown in Fig. 3, a cylindrical specimen is compressed between two Armco iron meter bars. The bars are gold-plated to minimize radiation losses, and the system is operated in a vacuum of $10^{-6}$ torr (a bell jar that fits over the entire apparatus is not shown) to minimize heat loss by gaseous convection and conduction. The bottom bar is threaded onto a heat sink cooled by temperature-controlled circulating water. A nichrome heater, driven by a temperature controller, is connected to the top bar. Calibrated chromel/constantan thermocouples are attached to the meter bars at regularly spaced intervals to monitor the temperature drops. By knowing the locations of the thermocouples and the temperatures they indicate, one can extrapolate to the temperatures at the two sample/indium/meter-bar interfaces and thus determine the heat flux. Series heat-flow measurements on test samples require a correction for the interfacial temperature drops. In the apparatus used, this correction is minimized by using optically flat surfaces (obtained by hand 


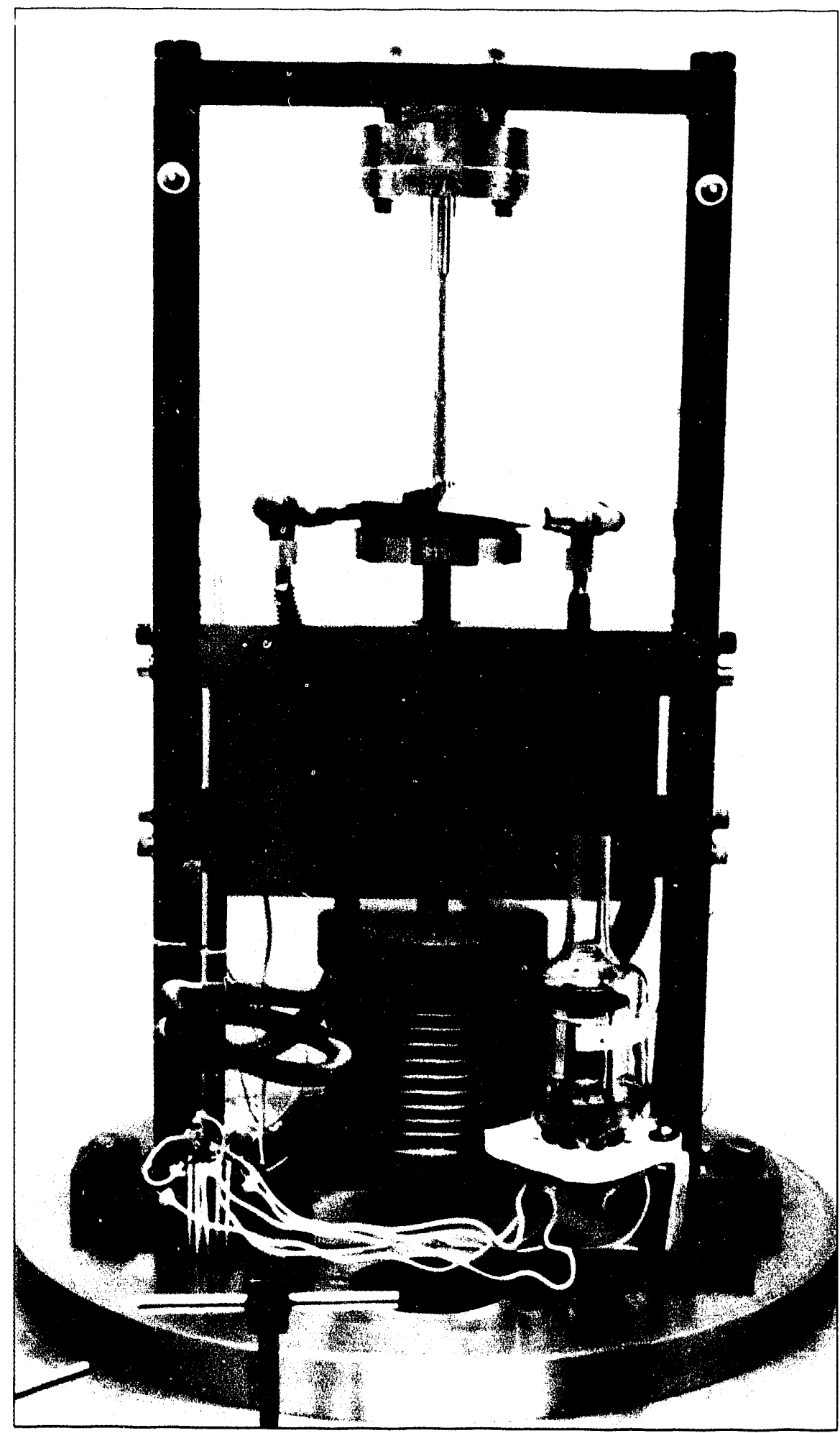

Fig. 3. Comparative longitudinal heat-flow apparatus. 
lapping), highly deformable indium foils at the interfaces, and contact pressures of about $34 \mathrm{MPa}^{3}$ Numerous other refinements and features are discussed in the literature. ${ }^{2,4}$

Operating the apparatus begins with aligning the specimen between the two meter bars and then applying a compressive load. An indium foil is placed at each specimen/meter-bar interface to minimize interfacial resistance. When compressed, the indium "flows" to fill in surface irregularities in the mating surfaces and thereby minimize the effects of varying surface finishes. After evacuating the system, the heater is energized, the water flow commences, and the system is allowed to reach a steady state (typically several hours). Taking data consists of reading the thermucouples in forward and reverse order. If radiation, convection, and gaseous conduction can be neglected, the heat flow through the meter bars is constant. Knowing the thickness of the sample permits calculating the thermal conductivity.

The total thermal resistance consists of contributions from the TP, the interfaces, and the Ni and In foils. Because the errors depend on the magnitudes of these four contributions to the total temperature drop, special methods are in order for highly insulating materials such as TPs. We used a stack of 24 elements like those shown in Fig. 4. Each element included a thin-film TP $\left(\mathrm{Y}_{2} \mathrm{O}_{3}: \mathrm{Eu}\right)$ sample deposited on a nickel substrate and an indium-foil interface to the next element, with an extra indium foil required at one end. This arrangement is the thermal analogue of the electrical measurement of the total resistance of a series string of resistors wherein the electrical resistances add in series. Here, the thermal resistances (reciprocals of the conductivities) are in series. The accuracy of the measured values for the TP obviously improves as its thermal resistance increases relative to the sum of the three correction terms.

Five of the $\mathrm{Y}_{2} \mathrm{O}_{3}$ :Eu samples are shown in Fig. 5, illuminated and excited by a UV lamp. The $\mathrm{Y}_{2} \mathrm{O}_{3}$ :Eu emits primarily at $611 \mathrm{~nm}$, in the orange-red region of the optical spectrum.

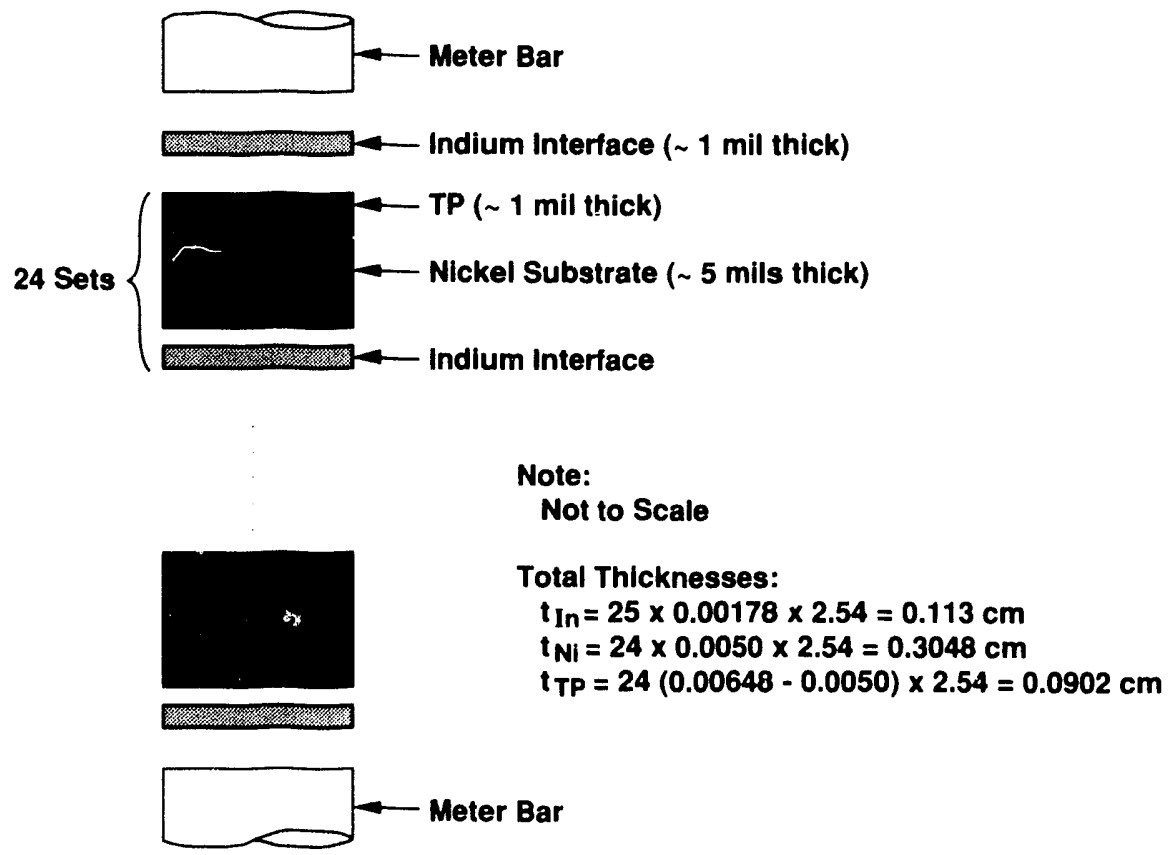

Fig. 4. Stack of elements that form a test sample for the heat-flow apparatus. 


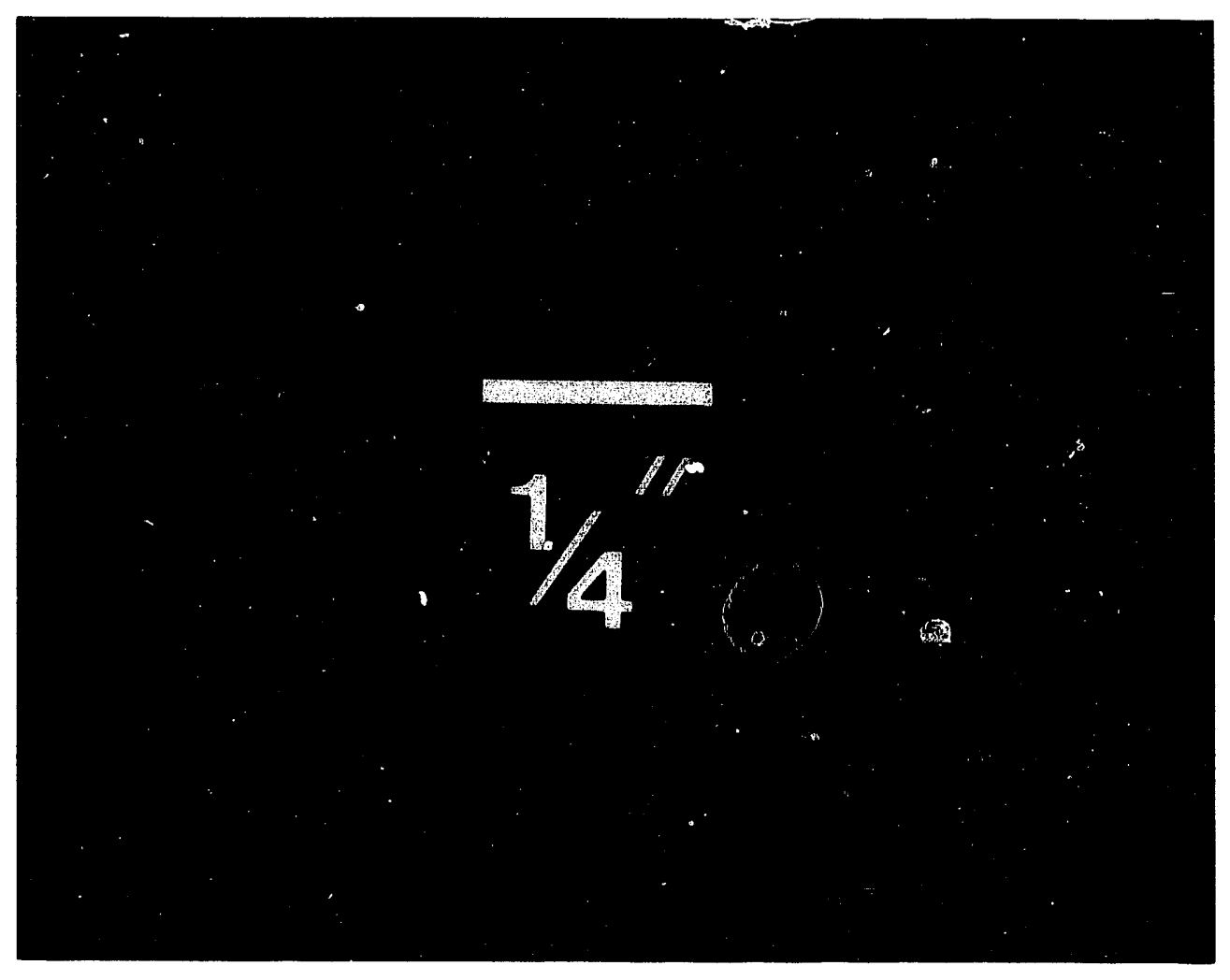

Fig. 5. Five of the nickel/TP elements used to make a test sample for the heat-flow apparatus. Here the samples are excited by UV light, so they emit fluorescence, which is primarily at $611 \mathrm{~nm}$.

Each indium foil was 1.78 mils thick. The TP was electron-beam-deposited onto 5-mil-thick nickel substrates. The TP thickness was determined by sectioning a spare sample and measuring the total sample thickness under a microscope, then subtracting the substrate thickness.

The thermal resistances of the nickel and indium are very small and are well known. Greater than $90 \%$ of the total resistance is in the TP coatings. The only other item of concern is the interface resistance. This was measured as a function of compressive stress to obtain a correction, which turned out to be negligible.

\section{B. Data and Analysis}

For a stack of elements of the different materials involved, the thermal conductivity of the unknown can be calculated from the formula relating the heat flux to the total thicknesses and the thermal conductivities,

$$
\frac{\Delta T}{q}=\frac{t_{I n}}{k_{I n}}+\frac{t_{N i}}{k_{N i}}+\frac{t_{T P}}{k_{T P}}+\frac{\Delta T_{i n t}}{q}
$$

where $\Delta T$ is the temperature drop between the meter bars, $q$ is the heat flux, $t_{i}$ is the total thickness of the samples of the $i^{\text {th }}$ material type, $k_{i}$ is the thermal conductivity of the $i^{\text {th }}$ material type, and $\Delta T_{i n t}$ is the tc nperature drop across the interfaces. The ratio $\Delta T / q$ and the thicknesses are measured; the thermal conductivities of $\mathrm{Ni}$ and In can be found in the literature; and, as pointed out earlier, the interface temperature drops are negligible; so the thermal conductivity of the TP can be calculated from the formula. Over the temperature range of interest, the thermal conductivity of $\mathrm{Ni}$ can be obtained from the empirical formula ${ }^{5}$

$$
k=1.291-0.00124 T,
$$


where $T$ is the temperature in kelvins, and the thermal conductivity of In can be calculated from the empirical formula ${ }^{6}$

$$
k=1.250-0.00112 T \text {. }
$$

We substituted the appropriate values of temperature into these formulas and combined the results with the experimental measurements of thickness and $\Delta T / q$ to form Table I. The results of the thermal conductivity measurements are plotted in Fig. 6. Note that the thermal conductivity of the $\mathrm{Y}_{2} \mathrm{O}_{3}: \mathrm{Eu}$ samples is nearly constant at $0.0137 \mathrm{~W} / \mathrm{cm} \cdot \mathrm{K}$ over the temperature range selected. The three experimental values varied by only $\pm 1.5 \%$.

TABLE I. Measurement of Thermal Conductivity of $\mathrm{Y}_{2} \mathrm{O}_{3}: \mathrm{Eu}$

\begin{tabular}{|c|c|c|c|c|}
\hline $\begin{array}{c}\mathrm{T}, \\
\text { Temperature, } \\
\mathrm{K} \\
\end{array}$ & $\begin{array}{c}\mathrm{k}_{I n}, \\
\text { t.c. of } \\
\text { indium, } \\
\mathrm{W} \cdot \mathrm{cm}^{-1} \cdot \mathrm{K}^{-1}\end{array}$ & $\begin{array}{c}k_{N i}, \\
\text { t.c. of } \\
\text { nickel } \\
\mathrm{W} \cdot \mathrm{cm}^{-1} \cdot \mathrm{K}^{-1} \\
\end{array}$ & $\begin{array}{c}\Delta T / q, \\
\mathrm{~W} \cdot \mathrm{cm}^{2} \cdot \mathrm{K}^{-1} \\
\end{array}$ & $\begin{array}{c}k_{t p} \\
\text { t.c. of } \\
\text { phosphor } \\
\mathrm{W} \cdot \mathrm{cm}^{-1} \cdot \mathrm{K}^{-1}\end{array}$ \\
\hline 303.2 & 0.7940 & 0.910 & 7.155 & 0.0135 \\
\hline 333.2 & 0.7709 & 0.877 & 6.982 & 0.0139 \\
\hline 363.2 & 0.7478 & 0.843 & 7.088 & 0.0137 \\
\hline
\end{tabular}

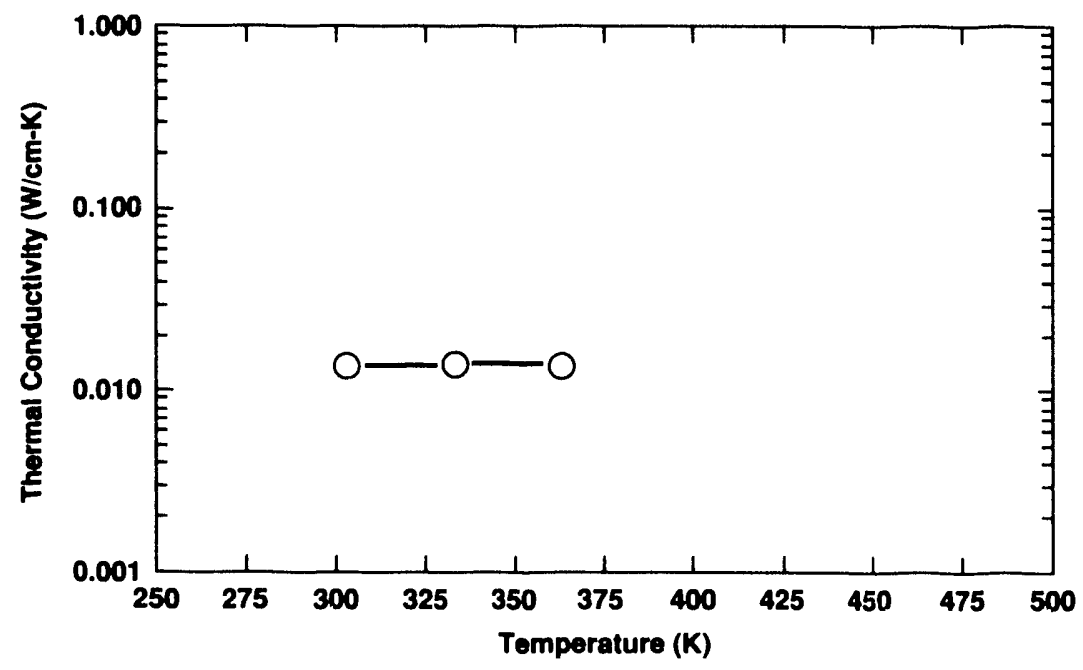

Fig. 6. Thermal conductivity of $\mathrm{Y}_{2} \mathrm{O}_{3}$ :Eu measured using a comparative longitudinal-heat-flow thermal-conductivity apparatus.

We did further analysis to check the reasonableness of these observations. An x-ray-diffraction scan of the $\mathrm{Ni}$, In, and $\mathrm{Y}_{2} \mathrm{O}_{3}$ :Eu composite showed no $\mathrm{Y}_{2} \mathrm{O}_{3}:$ Eu lines or unknown lines. Thus the crystallite size of the $\mathrm{Y}_{2} \mathrm{O}_{3}$ :Eu is sufficiently small that the TP appears to be amorphous. Therefore, we expect a small thermal conductivity that varies only slowly with temperature. The effect is similar to that of the grain size on the electrical conductivity; according to the kinetic theory, ${ }^{7}$ the 


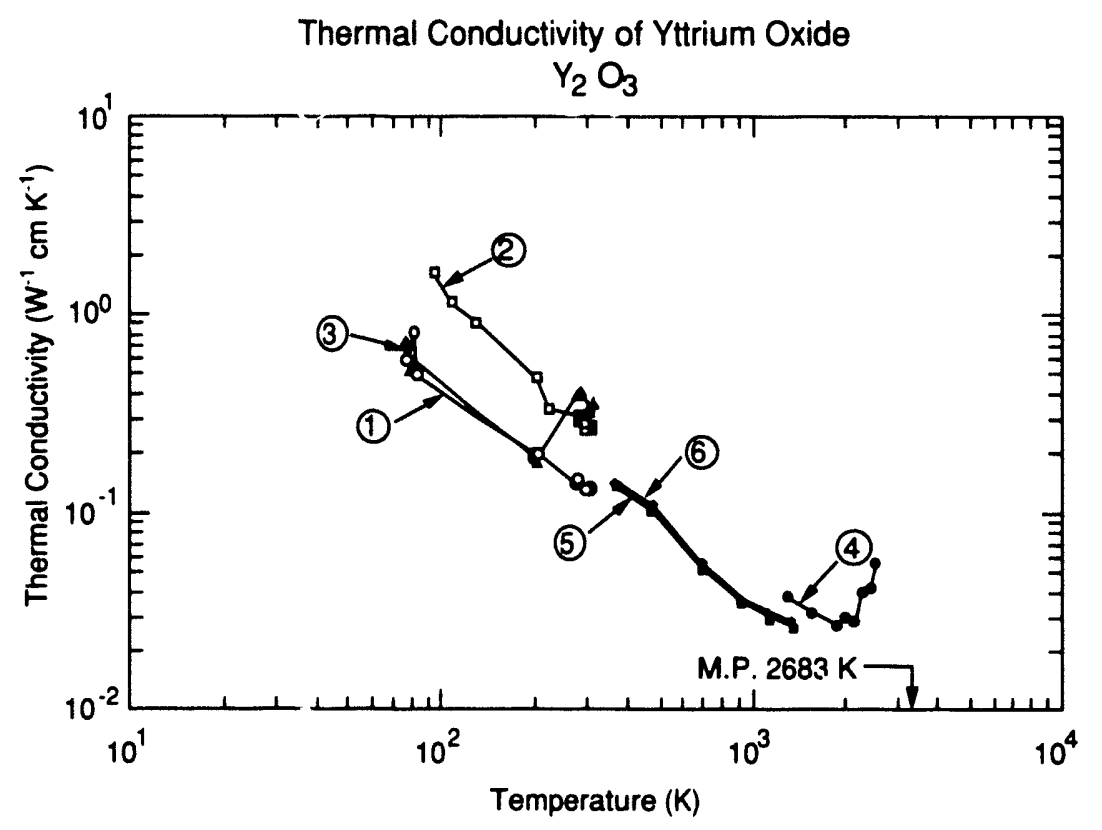

Fig. 7. Thermal conductivity of yttrium oxide (reproduced from Ref. 8). The six sets of data are for (1) single-crystal $\mathrm{Y}_{2} \mathrm{O}_{3}$ with $1.05 \% \mathrm{Nd}_{2} \mathrm{O}_{3} ;(2)$ single-crystal, high-purity $\mathrm{Y}_{2} \mathrm{O}_{3} ;(3)$ singlecrystal $\mathrm{Y}_{2} \mathrm{O}_{3}$ prepared from high purity naterial, but with $2.7 \times 10^{20} \mathrm{Nd}^{3+}$ atoms per $\mathrm{cm}^{3}$; (4) cold-pressed, high-purity $\mathrm{Y}_{2} \mathrm{O}_{3}$ powder; (5) dry-pressed, isostatically compacted, and sintered sample; and (6) the same specimen corrected for zero porosity. See Ref. 8 for more details.

total thermal conductivity is additively composed of the contributions from the various carriers that contribute to conduction (electrons, phonons, etc.), through the relation

$$
k=\frac{1}{3} \sum_{i}\left(\sigma_{i} \times V_{i} \times l_{i}\right),
$$

where the $i$ subscripts refer to the $i^{\text {th }}$ carrier type, $\sigma_{i}$ is the contribution of each carrier to the specific heat per unit volume, $V_{i}$ is the mean carrier velocity, and $l_{i}$ is the mean distance between collisions, or mean free path. In amorphous materials, the specific heat, carrier velocity, and carrier mean free path are essentially independent of temperature, so the thermal conductivity is also essentially constant within a factor of 4 or 5 . The mean free path is also very short in such materials at higher temperatures (above about $300 \mathrm{~K}$ ), so the thermal conductivity tends to be low.

We sought to compare the values of thermal conductivity that we obtained with any available in the literature. We knew from the outset that no directly comparable data exist; that very nonexistence was our motivation for doing the measurements. Data for pure $\mathrm{Y}_{2} \mathrm{O}_{3}$ (crystalline yttria) and $\mathrm{Y}_{2} \mathrm{O}_{3}$ with $1 \%$ dissolved $\mathrm{Nd}_{2} \mathrm{O}_{3}$ are reproduced in Fig. $7 .{ }^{8}$ Note that no data at all exist in the region of most interest to us, but the existing data are, nevertheless, informative. Neodymium and europium are similar rare earths; they are also both extensively soluble in $\mathrm{Y}_{2} \mathrm{O}_{3}$, so the thermal conductivity of yttria with either $\mathrm{Nd}$ or Eu dopants should be similar. As seen in the figure, the effect of the rare earth is dramatic; $1.05 \% \mathrm{Nd}_{2} \mathrm{O}_{3}$ reduces the thermal conductivity of yttria by a factor of 2 . Therefore, we might expect $6.8 \% \mathrm{Eu}_{2} \mathrm{O}_{3}$ to reduce the thermal conductivity to $\sim 0.02 \mathrm{~W} / \mathrm{cm} \cdot \mathrm{K}$ at $300 \mathrm{~K}$. This is within a factor of two of what we obtained. The low thermal conductivity values are also consistent with the kinetic theory, which predicts that the thermal conductivities of amorphous (or amorphous-like) materials are all low and do not vary much with temperature. 


\section{THEORY OF THE TP HEAT-FLUX GAUGE AND 2-D IMAGING}

In this section, we derive some useful expressions that will provide insight into the theoretical basis and the 2-D imaging of temperature distributions using the TP heat-flux gauges.

\section{A. Theoretical Basis of the Heat-Flux Gauge}

A thick aluminum substrate is coated with a thin uniform film of PMP. Buried in the PMP film are two layers of yttria particles located at two different depths from the surface. Although we are using a specific metal and plastic film, and specific types of particles, the analysis is general and many other combinations of substrate, film, and particles couid be used.

The outer surface of the PMP film is subjected to a thermal transient. If the thermal transient is slow, we expect the heat flux through the film to be given by the standard steady-state thermalconduction formula,

$$
q=K_{1} \frac{\left(T_{u}-T_{l}\right)}{d}
$$

where $K_{1}$ is the thermal conductivity of the PMP, $T_{u}$ is the measured temperature at the upper layer of yttria particles, and $T_{l}$ is the measured temperature at the lower layer of yttria particles. We use upper-case $K$ instead of lower-case $k$ for the thermal conductivity here to avoid confusion with the wave numbers that arise in the solution of the equations to follow.

For more-rapid transients, the the steady-state relationship will not be reached, and if we continue to use it to estimate the instantaneous heat flux during a rapid transient, some error will result or some correction will be required. This section is concerned with determining the actual temperatures and heat flux during a rapid thermal transient and using them to establish a correction to the steady-state formula.

1. No-Particle Solution. We begin by analyzing a thick aluminum substrate (region 1) coated with a thin film of PMP (region 2, of thickness $L$ ) without yttria particles. The transient temperature in the two regions is governed by the pair of equations

$$
\begin{aligned}
& \frac{\partial T_{1}}{\partial t}=\kappa_{1} \frac{\partial^{2} T_{1}}{\partial x^{2}} \text { for }-L<x<0 \\
& \frac{\partial T_{2}}{\partial t}=\kappa_{2} \frac{\partial^{2} T_{2}}{\partial x^{2}} \text { for } 0<x<\infty,
\end{aligned}
$$

where $x=0$ at the interface between regions 1 and 2 and the proportionality constants $\kappa_{i}$ are, as usual, $\kappa_{i}=K_{i} / \sigma_{i} \rho_{i}$, in which $K_{i}$ is the thermal conductivity, $\sigma_{i}$ is the specific heat, and $\rho_{i}$ is the material density.

We seek solutions of the form

In region 1 ,

$$
T \sim \exp i(k x-\omega t)
$$

$$
\begin{aligned}
-i \omega & =\kappa_{1}\left(i k_{1}\right)^{2}=-\kappa_{1} k_{1}^{2}, \\
k_{1} & = \pm\left(\frac{i \omega}{\kappa_{1}}\right)^{1 / 2},
\end{aligned}
$$


with the convention that the square root has a positive real part. A similar solution can be obtained for region 2. The general solution can then be written as the sum of incoming and outgoing temperature waves in each layer:

$$
\begin{aligned}
& T_{1}=A \exp i\left(k_{1} x-\omega t\right)+B \exp i\left(-k_{1} x-\omega t\right) \\
& T_{2}=C \exp i\left(k_{2} x-\omega t\right)+D \exp i\left(-k_{2} x-\omega t\right) .
\end{aligned}
$$

The substrate layer is assumed to be so thick that there is no reflected wave from its far surface, so $D=0$. The free surface of the PMP layer, $x=-L$, is assumed to be exposed to an externally applied heat flux that is harmonic in time:

$$
-K_{1} \frac{\partial T_{1}}{\partial x}=Q_{0} \exp -i \omega t
$$

where $K_{1}$ is the thermal conductivity of the PMP. Substituting,

$$
i K_{1} k_{1} A \exp -i k_{1} L-i K_{1} k_{1} B \exp +i k_{1} L=-Q_{0} \text {. }
$$

At the interface between the PMP layer and the substrate $(x=0)$, the temperature and the heat flux are continuous:

$$
\begin{aligned}
A+B & =C \\
K_{1} k_{1} A-K_{1} k_{1} B & =K_{2} k_{2} C
\end{aligned}
$$

where $K_{2}$ is the thermal conductivity of the substrate. Combining the three equations, we find that

$$
\begin{aligned}
& A=\frac{\frac{Q_{0} \exp -i k_{1} L}{K_{1} k_{1}}}{\frac{K_{2} k_{2}-K_{1} k_{1}}{K_{1} k_{2}+K_{1} k_{1}}+\exp -2 i k_{1} L} \\
& B=-\frac{K_{2} k_{2}-K_{1} k_{1}}{K_{2} k_{2}+K_{1} k_{1}} A \\
& C=A+B .
\end{aligned}
$$

2. Single-Sphere Plane-Wave Scattering. We now consider the effect of placing in the PMP layer a single, isolated yttria sphere, which then interacts with the thermal wave.

We assume a unit incident plane temperature wave of the form

$$
T_{1}=\exp i\left(k_{1} x-\omega t\right)
$$

where (to solve the heat equation) the wave number must be

$$
k_{1}=\left(\frac{i \omega}{\kappa_{1}}\right)^{1 / 2}
$$


Outside of the sphere, we assume a scattered spherical wave of the form

$$
T_{2}=\sum_{n} B_{n} P_{n}(\theta) h_{n}^{(1)}\left(k_{2} r\right) \exp -i \omega t
$$

where the $B_{i}, P_{i}$, and $h_{i}$ are to be determined. The temperature wave $T_{2}$ must satisfy the spherical heat equation,

$$
\frac{\partial T}{\partial t}=\kappa_{2} \frac{1}{r^{2}} \frac{\partial}{\partial r} \frac{\partial T}{\partial r}+\kappa_{2} \frac{1}{r^{2} \sin \theta} \frac{\partial}{\partial \theta}\left(\sin \theta \frac{\partial T}{\partial \theta}\right) .
$$

Substituting, we obtain

$$
-i \omega B_{n} P_{n}(\theta) h_{n}\left(k_{n} r\right)=B_{n} P_{n}(\theta) \kappa_{2} \frac{1}{r^{2}} \frac{\partial}{\partial r} r^{2} \frac{\partial h_{n}^{(1)}}{\partial r}+B_{n} h_{n}^{(1)}\left(k_{n} r\right) \kappa_{2} \frac{1}{r^{2} \sin \theta} \frac{\partial}{\partial \theta}\left(\sin \theta \frac{\partial P_{n}}{\partial \theta}\right)
$$

or

$$
-i \omega=\frac{\kappa_{2}}{h} \frac{1}{r^{2}} \frac{\partial}{\partial r} r^{2} \frac{\partial h}{\partial r}+\frac{\kappa_{2}}{P_{n}} \frac{1}{r^{2} \sin \theta} \frac{\partial}{\partial \theta}\left(\sin \theta \frac{\partial P_{n}}{\partial \theta}\right) .
$$

We know from the definition of Legendre's polynomials, $P_{n}$, that

$$
\frac{1}{P_{n}} \frac{1}{\sin \theta} \frac{\partial}{\partial \theta}\left(\sin \theta \frac{\partial P_{n}}{\partial \theta}\right)=-n(n+1) .
$$

Separation of variables gives, for $h$,

$$
\frac{1}{r^{2}} \frac{\partial}{\partial r} r^{2} \frac{\partial h}{\partial r}+\left[-\frac{n(n+1)}{r^{2}}+\frac{i \omega}{\kappa_{2}}\right] h=0 .
$$

According to Abramowitz and Stegun, spherical Bessel functions satisfy

$$
r^{2} w^{\prime \prime}+2 r w^{\prime}+\left[r^{2}-n(n+1)\right] w=0 .
$$

Thus, $h_{n}$ is a spherical Bessel function with $k_{2}=\left(i \omega / \kappa_{2}\right)^{1 / 2}=k_{1}=\left(i \omega / \kappa_{1}\right)^{1 / 2}$. Also, $h_{n}^{(1)}$ satisfies an outgoing-wave boundary condition.

On the other hand, inside the sphere, we assume the form

$$
T_{3}=\sum_{n} D_{n} P_{n}(\theta) j_{n}\left(\kappa_{3} r\right) \exp -i \omega t
$$

We find, in the same way, that $j_{n}$ is a spherical Bessel function, with $k_{3}=\left(i \omega / \kappa_{3}\right)^{1 / 2}$. Also, $j_{n}$ is bounded at the origin.

At the surface of the sphere, $r=a$, the temperature and radial heat flux are continuous. Letting $x=a \cos \theta$, we then have

$$
\exp i k_{1} a \cos \theta+\sum_{n} B_{n} P_{n}(\cos \theta) h_{n}^{(1)}\left(k_{1} a\right)=\sum_{n} D_{n} P_{n}(\cos \theta) j_{n}\left(k_{3} a\right)
$$

and

$$
K_{1}\left[i k_{1} \cos \theta \exp i k_{1} a \cos \theta\right]+K_{1} \sum_{n} B_{n} P_{n}(\cos \theta) k_{1} h_{n}^{(1)^{\prime}}\left(k_{1} a\right)=K_{3} \sum_{n} D_{n} P_{n}(\cos \theta) k_{1} h_{n}^{(1) \prime}\left(k_{3} a\right)
$$


Multiplying both sides by $P_{m}(\cos \theta)$, letting $\cos \theta=x$, and integrating, we find that

$$
\begin{gathered}
D_{m} N_{m} j_{m}\left(k_{3} a\right)-B_{m} N_{m} h_{m}^{(1)}\left(k_{1} a\right)=\int_{-1}^{1} P_{m}(x) \exp i k_{1} a x d x \\
K_{3} D_{m} N_{m} k_{3} j_{m}^{\prime}\left(k_{3} a\right)-K_{1} B_{m} N_{m} k_{1} h_{m}^{(1))^{\prime}}\left(k_{1} a\right)=K_{1} \int_{-1}^{1} i k_{1} P_{m}(x) x \exp i k_{1} a x d x,
\end{gathered}
$$

where $N_{m}$ is the normalization constant for the Legendre polynomials.

The above two equations require evaluating certain integrals involving Legendre polynomials and exponentials. These integrals may be evaluated by repeated integration by parts, integrating the exponential and differentiating the polynomial until the order of the polynomial becomes zero. We carried out this scheme, programmed it in FORTRAN, and tested it, but found that more accurate results can be obtained by evaluating the integrals approximately by Gaussian quadrature rather than by using the "exact" formulas obtained with integration by parts.

We wrote a FORTRAN program to Fourier-transform an observed temperature signal, convert each Fourier component into an incoming heat flux, and invert the Fourier transform to obtain a time histogram of the incoming heat flux.

For the case of many yttria particles in each layer, we make the simplifying approximations that (1) the particles are sufficiently far from one another, and from other boundaries, that the scattered thermal wave from one particle does not significantly affect the temperature of other particles, and (2) that a thermal wave scattered from a particle does not interact with a material interface such that it reflects back with enough intensity to significantly affect the temperature of the scattering particle.

3. Calibration. The calibration technique used for the MIT gauge ${ }^{9}$ discussed in our previous report ${ }^{1}$ was very simple and effective. It should be easier to calibrate than the TP gauge, partly because it makes use of temperature measurements precisely at the surface, and partly because it involves fewer parameters. The TP gauge does not directly determine the PMP surface temperature. It also introduces new parameters: the TP particle diameter; two mean particle depths and distribution of depths; the particle spacing in a plane parallel to the surface, and the $\mathrm{TP}$ thermal conductivity and specific heat.

One possible approach to calibration is to apply a pulse of heat-flux input (of nearly zero or at least the smallest feasible duration), and to measure the response of each TP-bead layer separately. Then, by nonlinear least squares, try to adjust the model parameters to give the best fit to both pulse responses. It seems likely that such a calibration procedure could at least be used to back out the two particle-layer depths. There is a small possibility that some additional calibration information could be obtained by least squares. It seems extremely unlikely that all of the data associated with the TP heat-flux gauge could be calibrated out.

The adjusted model would be used to generate a nominal heat-flux pulse response. The pulse response would be Fourier-transformed. The transform would be inverted and truncated at some high frequency. Perhaps the least-squares fitting should be performed on the truncated pulse response.

To process real data, the measured nominal heat flux would be Fourier-transformed, multiplied by the truncated-inverted pulse response, and then Fourier-transformed again. 
We compared $8-\mu \mathrm{m}$ spheres with $2-\mu \mathrm{m}$ spheres using the same center depths. There was little difference $(\approx 1$ or $2 \%)$ in the Fourier-synthesized particle-response curves. Thus, little difference is expected between $8-\mu \mathrm{m}$ spheres and no spheres at all if the no-sphere temperature is taken at the sphere center depth, so no-sphere calculations might be used for a calibration of a gauge with $8-\mu \mathrm{m}$ spheres. Note, however, that some of our gauges use much larger clusters of particles. The discussion here probably does not apply to those gauges.

Figure 8 is a plot of the computed time- $v s$-temperature response of TP beads embedded just under the surfaces of the two faces of a PMP insulator. A unit heat flux impinges on the top surface and gives the rapid peak response of the upper TP. As can be seen, the gauge responds quickly. It then reaches equilibrium in about one unit of time, when another pulse could be applied. Two different time responses are evident: a very fast one, in which the gauge, with no temperature difference across it, responds to a pulse of heat flux; and a second, somewhat slower one, that determines the rate at which pulses can be applied.

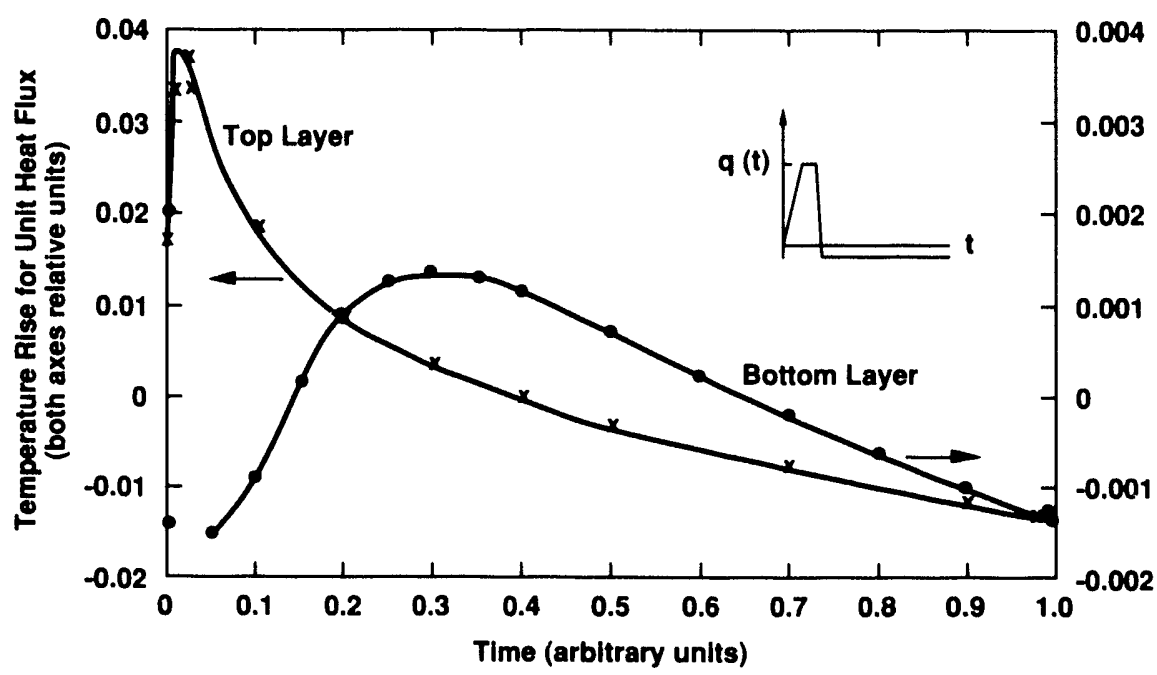

Fig. 8. Theoretical pulse response of TP beads in a plastic substrate. The curve fits are to the indicated calculated data points.

\section{B. 2-D Imaging Theory}

1. Ratio Method. The ability to remove geometric distortion and illumination variations from spectrally selected data is shown here by presenting a model of imaging. This model demonstrates some of the theoretical limitations of the ratio method and is the foundation for implementing the logarithms necessary to extract surface temperature. We point out in §V.B that further manipulation is needed to perform the same process with heat-flux gauges. Figure 9 represents the imaging geometry and the associated nomenclature. The following definitions are pertinent to the figure.

$$
\begin{aligned}
\delta O_{1}= & \text { fluorescent-emission radiance emanating from an infinitesimal surface patch on the } \\
& \text { object at wavelength } \lambda \\
\delta I_{\lambda}= & \begin{array}{l}
\text { irradiance of emission photons onto the image plane, i.e., the imaged infinitesimal } \\
\text { object patch at wavelength } \lambda
\end{array}
\end{aligned}
$$


$t_{\lambda}=$ narrow-band transmission fraction of the optics to wavelength $\lambda$

$\alpha=$ angle that an emission ray passing through the optics makes with the optical axis, $z$

$\theta=$ angle that the emission ray makes with the surface normal, $\hat{n}$, associated with the object patch

$(\theta, \phi)=$ polar and azimuthal angles associated with the surface normal, $\hat{n}$

$f=$ fixed distance from the image plane to the lens

$(x, y)=$ coordinates of a point in the image plane

$\left(x^{\prime}, y^{\prime}, z\right)=$ coordinates of a point on the object surface

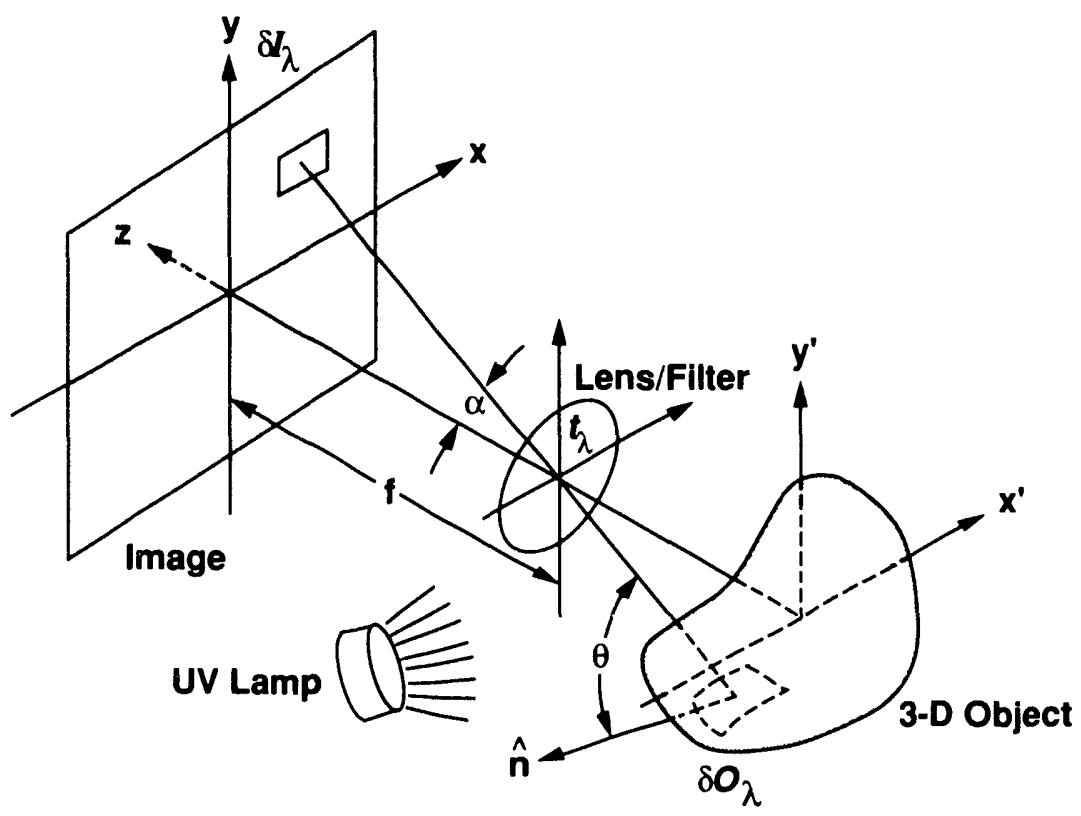

Fig. 9. Coordinate system and nomenclature used for development of imaging model.

We wish to obtain an expression for the distribution of object fluorescence at the image plane. We begin by describing the radiance from an infinitesimal object patch and how it interacts with the imaging plane to create an infinitesimal image patch. The irradiance of the image patch arising from the radiance (fluorescence) of the object patch is ${ }^{10}$

$$
\delta I_{\lambda}(x, y)=t_{\lambda} \delta O_{\lambda}\left(x^{\prime}, y^{\prime}, z\right)\left(\frac{f}{z}\right) \frac{\cos \theta}{\cos \alpha}
$$

where the transformation from object coordinates $\left(x^{\prime}, y^{\prime}, z\right)$ to image-plane coordinates $(x, y)$ is given by $x=(f / z) x^{\prime}$ and $y=(f / z) y^{\prime}$. We assume that the object surface-depth coordinate, $z$, varies only slightly with respect to the distance from the image plane; i.e., the ratio $f / z$ can be approximated by a constant. 
To understand the illumination and geometry responses of the system, we derive the dependence of $\delta I_{\lambda}(r, y)$ on the direction coordinates $(\theta, \phi)$ of the incident excitation and excitant emission. Let the solid angles defined by the incoming and excitant radiation be $\Omega_{i}=\left(\theta_{i}, \phi_{i}\right)$ and $\Omega_{e}=\left(\theta_{e}, \phi_{e}\right)$, respectively. The temperature-dependent object radiance, $\delta O_{\lambda}\left(x^{\prime}, y^{\prime}, z, T, \Omega_{e}\right)$ for emission at wavelength $\lambda$, is then written as

$$
\delta O_{\lambda}\left(x^{\prime}, y^{\prime}, z, T ; \Omega_{e}\right)=\int_{\Omega_{i}} f_{\lambda}\left(x^{\prime}, y^{\prime}, z, T ; \Omega_{i} \Omega_{e}\right) u\left(x^{\prime}, y^{\prime}, z ; \Omega_{i}\right) \cos \theta_{i} d \Omega_{i}
$$

where $u\left(x^{\prime}, y^{\prime}, z ; \Omega_{i}\right)$ is the direction-dependent distribution of the incident UV excitation (i.e., the source distribution) at all incident angles $\Omega_{i}$. Note that $d \Omega_{i}=\sin \theta_{i} d \theta_{i} d \phi_{i}$ is the solid angle subtended by the object patch and the UV source. The term $\cos \theta_{i}$ corrects the surface-patch distribution for tilt of the surface normal, $\hat{n}$, relative to the incoming excitation source. The function $f_{\lambda}\left(x^{\prime}, y^{\prime}, z, T ; \Omega_{i}, \Omega_{e}\right)$ is the temperature-modulated spatial distribution of emission photons (i.e., the fluorescence distribution) at wavelength $\lambda$. It contains the spatial and thermal information associated with a TP-coated surface.

The TP-coated object can be approximated as a Lambertian emitter because an absorbed UV photon will be re-emitted as a visible photon with random direction. Therefore, the direction of an emitted photon (surface radiance), at wavelength $\lambda$, caused by the irradiart UV photon from the excitation source is uncorrelated and the dependency of $f_{\lambda}$ can be simplified:

$$
f_{\lambda}\left(x^{\prime}, y^{\prime}, z, T ; \Omega_{i}, \Omega_{e}\right) \rightarrow f_{\lambda}\left(x^{\prime}, y^{\prime}, z, T ; \Omega_{e}\right)
$$

The fluorescence distribution can be modeled as a separable function with angle-dependent and amplitude components, i.e., the distribution is amplitude-modulated as the energy of the incident UV photon is preferentially shared between electron and phonon excitation in the material lattice. ${ }^{11}$ The amplitude component, $L$, of the separable function also contains the wavelength dependence of the fluorescence,

$$
f_{\lambda}\left(x^{\prime}, y^{\prime}, z, T ; \Omega_{e}\right)=f\left(x^{\prime}, y^{\prime}, z ; \Omega_{e}\right) L_{\lambda}\left[T\left(x^{\prime}, y^{\prime} z\right)\right] .
$$

This result may be substituted into the integral and the coordinates $\left(x^{\prime}, y^{\prime}, z\right)$ transformed to $(x, y)$ with the transformations given above. This gives the infinitesimal object-patch emission distribution as a function of image-plane coordinates from which we can readily derive the imageplane distribution. The object-patch distribution is

$$
\begin{aligned}
\delta O_{\lambda}\left(x, y, T ; \Omega_{e}\right) & =\int_{\Omega_{i}} f\left(x, y, \Omega_{e}\right) L_{\lambda}[T(x, y)] u\left(x, y ; \Omega_{i}\right) \cos \theta_{i} d \Omega_{i} \\
& =f\left(x, y ; \Omega_{e}\right) L_{\lambda}[T(x, y)] \int_{\Omega_{i}} u\left(x, y ; \Omega_{i}\right) \cos \theta_{i} d \Omega_{i} \\
& =f\left(x, y ; \Omega_{e}\right) L_{\lambda}[T(x, y)] U(x, y),
\end{aligned}
$$

where $U(x, y)$ is the geometry and amplitude function that defines the excitation source. It is a constant at each spatial point $(x, y)$ until the source is relocated or changes in intensity. We now combine this result with the first expression, relating the irradiance of the image patch that arises 
from the object patch, to reveal the image-patch distribution, $\delta I_{\lambda}$, arising from the object-patch emission, $\delta O_{\lambda}$,

$$
\delta I_{\lambda}\left(x, y, T ; \Omega_{e}\right)=t_{\lambda} f\left(x, y ; \Omega_{e}\right) L_{\lambda}[T(x, y)] U(x, y)\left(\frac{f}{z}\right)^{2} \frac{\cos \theta}{\cos \alpha}
$$

The imaged intensity at any point on the image plane, $(x, y)$, caused by emission at object point $\left(x^{\prime}, y^{\prime}, z\right)$ is determined by integrating over all emission angles, $\Omega_{e}$, that are within the collection field of the optics; i.e.,

$$
\begin{aligned}
I_{\lambda}(x, y, T) & =\int_{\Omega_{e}} \delta I_{\lambda}\left(x, y, T ; \Omega_{e}\right) \\
& =\int_{\Omega_{e}} t_{\lambda} f\left(x, y ; \Omega_{e}\right) L_{\lambda}[T(x, y)] U(x, y)\left(\frac{f}{z}\right)^{2} \frac{\cos \theta}{\cos \alpha} d \Omega_{e} \\
& =t_{\lambda} L_{\lambda}\left[(T(x, y)] U(x, y) \int_{\Omega_{e}} f\left(x, y ; \Omega_{e}\right)\left(\frac{f}{z}\right)^{2} \frac{\cos \theta}{\cos \alpha} d \Omega_{e}\right. \\
& =t_{\lambda} L_{\lambda}[T(x, y)] U(x, y) E(x, y),
\end{aligned}
$$

where $E(x, y)$ defines the object-emission geometry at each surface point, $(x, y)$. Note that this final result represents the imaged distribution of emission photons as a separable function of excitation geometry, excitation amplitude, emission geometry, and modulated temperaturedependent emission amplitude.

2. Temperature. To determine the surface temperature, we form the ratio of two spectrally filtered images at known wavelengths $\left(\lambda_{1}, \lambda_{2}\right)$. In the absence of background, the ratio will have a spatial and temporal dependence denoted by

$$
r(x, y, T)=\frac{I_{\lambda_{1}}(x, y, T)}{I_{\lambda_{2}}(x, y, T)}=\frac{t_{\lambda_{1}} L_{\lambda_{1}}[T(x, y)]}{t_{\lambda_{2}} L_{\lambda_{2}}[T(x, y)]} .
$$

Note that the geometry dependence on object illumination, $U(x, y)$, and the fluorescent emission, $E(x, y)$, cancel, leaving only a modulated amplitude response, which is directly proportional to the surface temperature at all imaged points $(x, y)$. The surface temperature is extracted by empirical determination of a transform function, $\mathcal{F}\{\}$, that operates on the ratio,

$$
T(x, y)=\mathcal{F}\left\{\frac{t_{\lambda_{2}}}{t_{\lambda_{1}}}\right\} r(x, y, T)
$$

Turley et al. ${ }^{1}$ demonstrated that the response of the emission ratio for $\mathrm{Gd}_{2} \mathrm{O}_{2} \mathrm{~S}: \mathrm{Tb}$ at $415 \mathrm{~nm}$ and $490 \mathrm{~nm}$ is linear over the temperature range of approximately 50 to $120^{\circ} \mathrm{F}$. This linear response was verified for our existing video system. The transformation function that applies to the ratioed image is defined for the linear system by

$$
T(x, y)=\mathcal{F}\{r\}=T_{h}-\left(T_{h}-T_{l}\right) \frac{\left(r-r_{l}\right)}{\left(r_{h}-r_{l}\right)},
$$


where $\left(T_{h}, T_{l}\right)$ and $\left(r_{h}, r_{l}\right)$ are, respectively, the high and low limits of the TP temperature and ratio calibration.

\section{IMAGING TEST}

We did a simple preliminary imaging test to discover what problems were inherent in the method. We had previously demonstrated that it is possible to obtain 2-D maps of the temperature distribution on TP-coated turbine blades. ${ }^{12}$

\section{A. Experiment Setup}

A heat-flux gauge of the type described in $\S I I$ was fabricated using triangular patterns of $\mathrm{La}_{2} \mathrm{O}_{2} \mathrm{~S}: \mathrm{Eu}$ and $\mathrm{Gd}_{2} \mathrm{O}_{2} \mathrm{~S}$ :Tb TPs. The gauge was attached to a turbine blade and mounted on a test fixture, as shown in Figs. 10 through 12. This is the same heat-flux gauge that is shown close up in Fig. 2.

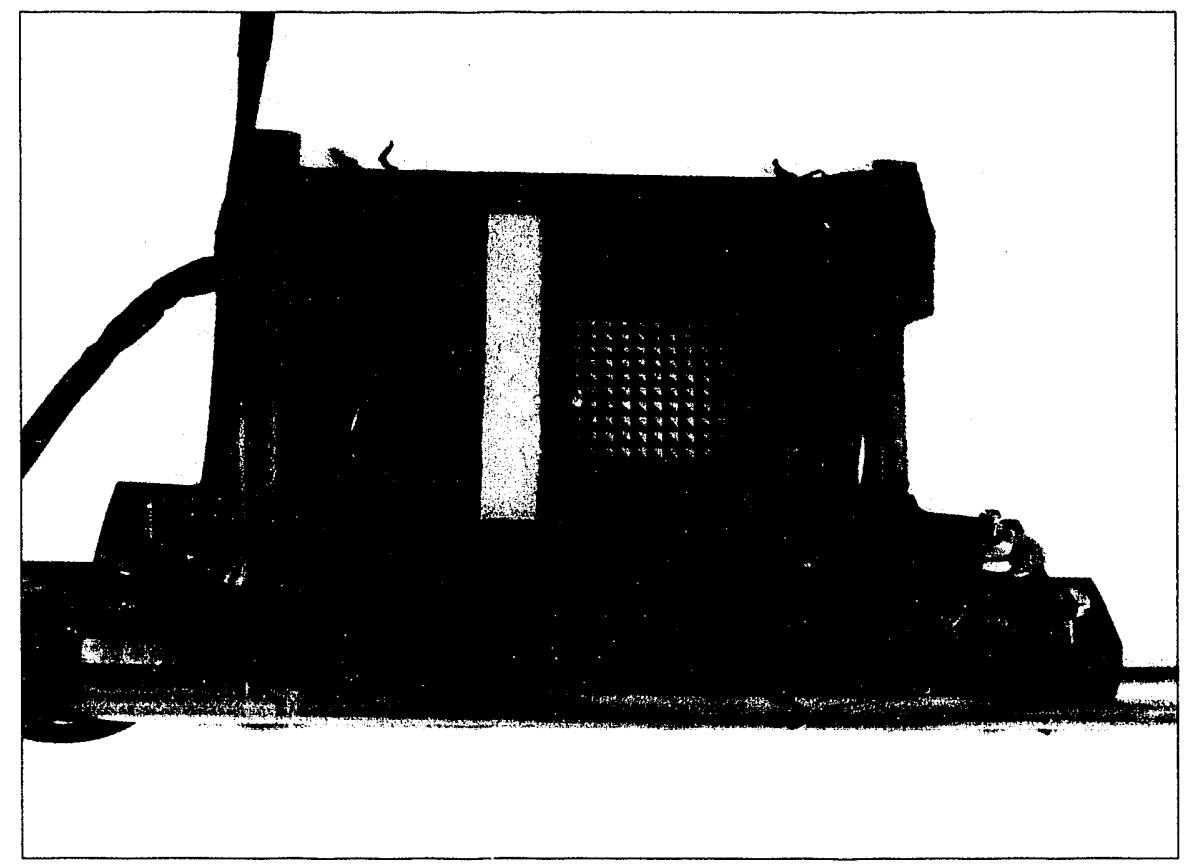

Fig. 10. Gauge mounted on a turbine blade and not illuminated by a UV lamp.

Figure 10 shows the gauge mounted on the blade and Fig. 11 shows it mounted on the blade and illuminated by the UV lamp. Figure 12 shows the back side of the blade. Four holes were drilled through the blade and thermocouples were inserted through the holes from back to front so that their tips were flush with the front surface. We expected to use the temperatures measured by the thermocouples as references for the gray-scale and false-color images. As it turned out, the test was done only at room temperature because of insurmountable problems with the data acquisition and analysis, so the thermocouples were not used.

Figure 13 shows the experiment arrangement. The gauge and test fixture were mounted so they could be viewed by a video camera. A heater element was attached to the back side of the blade to create a temperature gradient on the blade. A UV lamp illuminated the heat-flux gauge. The 


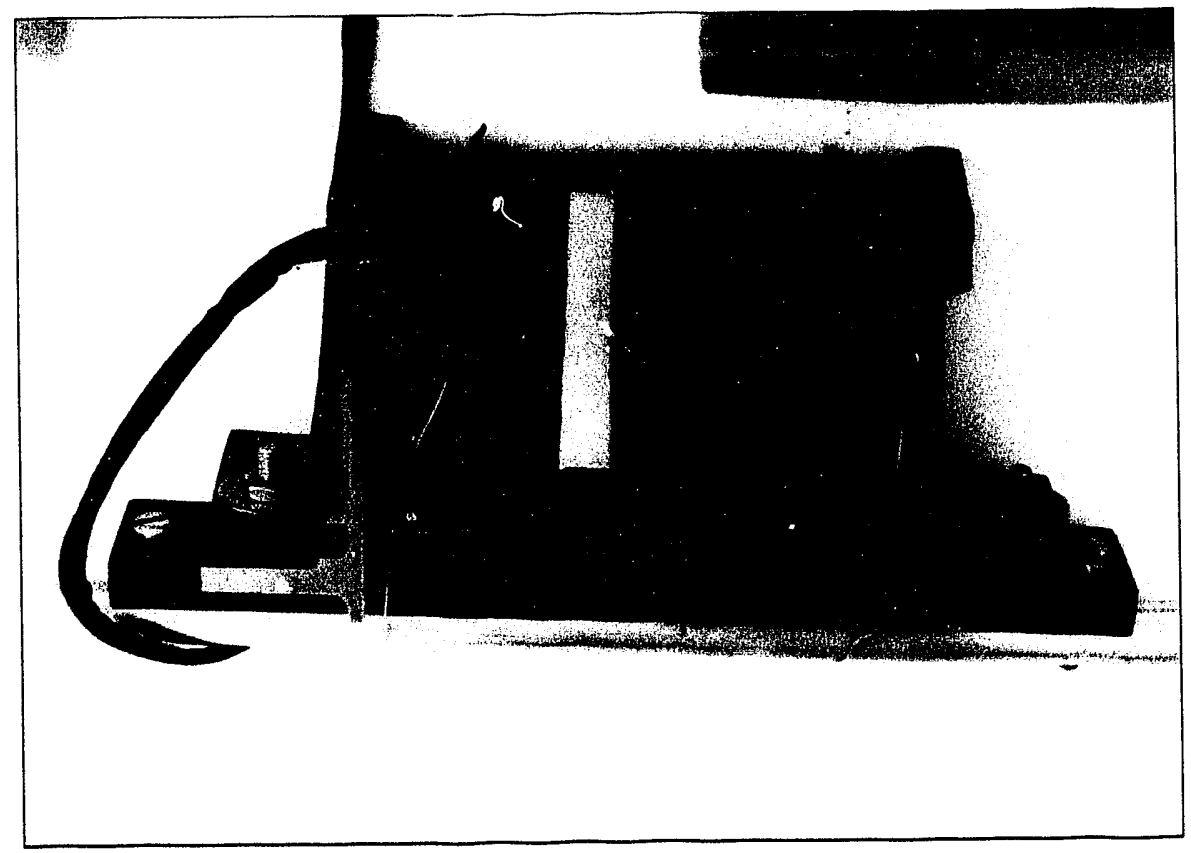

Fig. 11. Gauge mounted on a turbine blade and excited by a UV lamp. The $\mathrm{Gd}_{2} \mathrm{O}_{2} \mathrm{~S}$ :Tb shows up as green and the $\mathrm{La}_{2} \mathrm{O}_{2} \mathrm{~S}: \mathrm{Eu}$ as yellow.

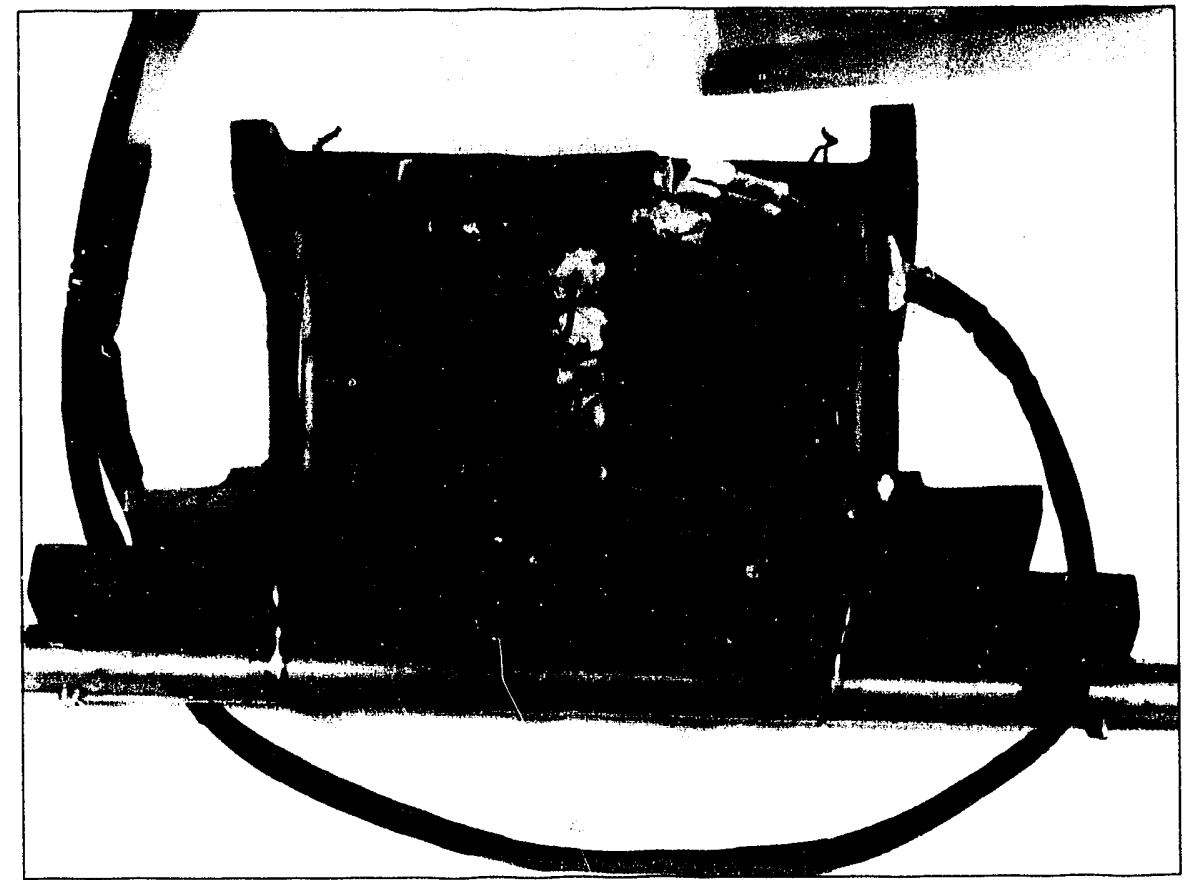

Fig. 12. Back side of turbine blade, showing thermocouples.

light emitted by the TPs passed through appropriate filters and into an RCA TC1040H intensified silicon-intensified-target-vidicon closed-circuit-television camera. The filters were chosen to select the lines necessary for determining the amplitude ratios. For the $\mathrm{Gd}_{2} \mathrm{O}_{2} \mathrm{~S}: \mathrm{Tb}$, the background and temperature-sensitive lines are at 490 and $415 \mathrm{~nm}$, respectively. For the $\mathrm{La}_{2} \mathrm{O}_{2} \mathrm{~S}: \mathrm{Eu}$, the 
corresponding lines are at 614 and $511 \mathrm{~nm}$. The four filters were centered at the same wavelengths and were $10 \mathrm{~nm}$ wide. Figure 14, taken from Reference 1, shows these emission lines on the combined spectra of $\mathrm{Gd}_{2} \mathrm{O}_{2} \mathrm{~S}: \mathrm{Tb}$ and $\mathrm{La}_{2} \mathrm{O}_{2} \mathrm{~S}: \mathrm{Eu}$. The video camera has been extensively modified to convert it to a data-acquisition camera. The video output from the camera was digitized to 8-bit precision using a Data Translation DT2861 frame grabber and a DT 7020 32-bit floating-point array processor. The process was controlled by the host 80286-processor-based persunal computer. The image data were displayed on a standard 512 by 512 RGB color monitor.

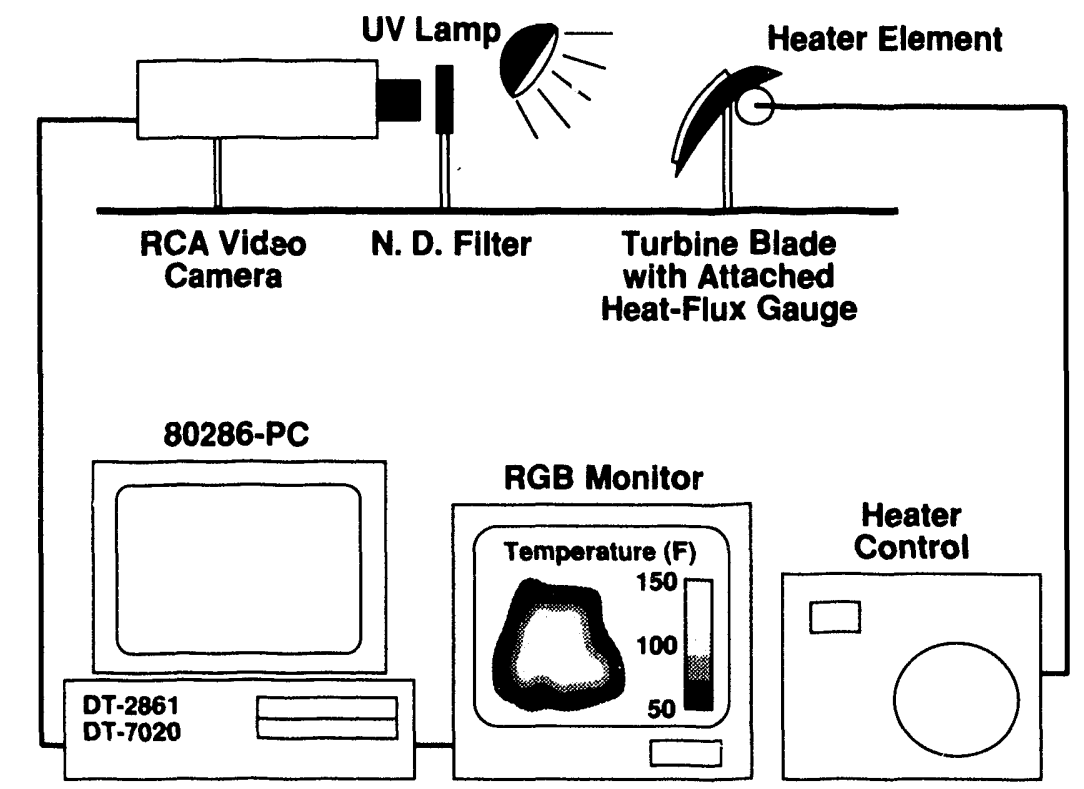

Fig. 13. Experiment setup for demonstration of heat-flux-gauge imaging.

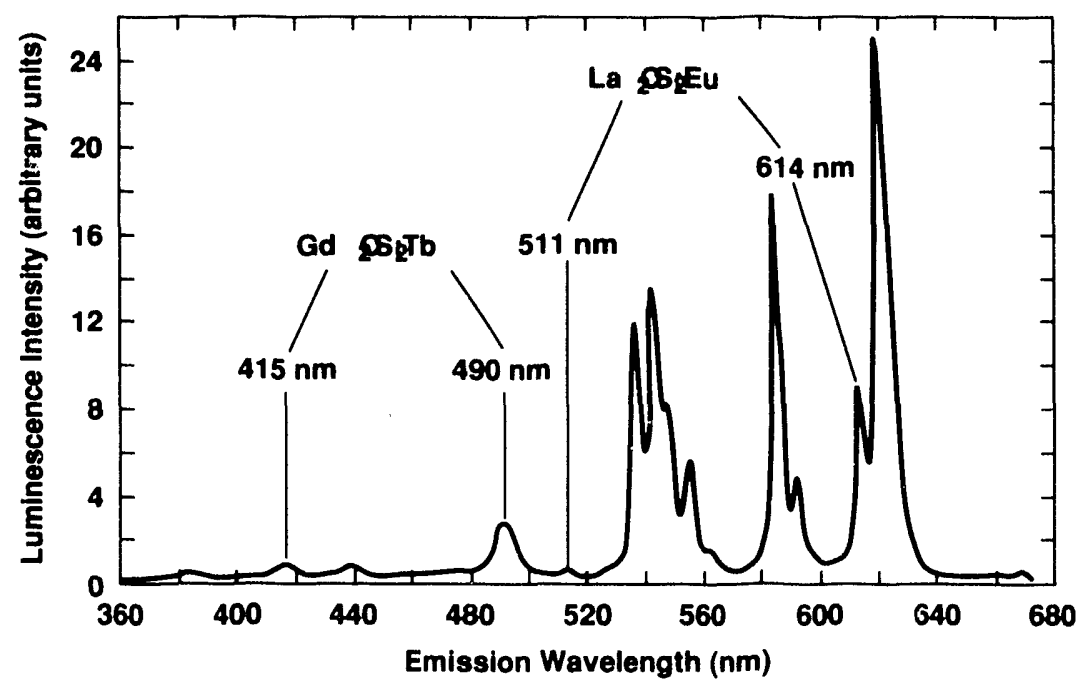

Fig. 14. Li minescence spectrum of a prototype heat-flux gauge at $51^{\circ} \mathrm{F}$ (from Reference 1). 


\section{B. Data and Analysis}

Data were obtained for the four possible images at room temperature. Our attempts to process the data in a meaningful way that would result in a 2-D map of either a temperature distribution or a heat-flux distribution were frustrated by a number of inadequacies in commercial hardware. It was purchased for our earlier work on imaging 2-D temperature distributions and is effective for many operations on 2-D arrays. For example, it allows fast retrieval and storage of information from the camera and host computer, and it can rapidly perform arithmetic-array operations such as add, subtract, Boolean logic, and look-up-table mapping of grey-scale information. But the hardware cannot do more-complex operations, such as morphological processing, spatial filtering (e.g., convolution), and nondestructive graphic overlay. It is therefore too basic to permit doing some of the necessary tasks within the available time (and, therefore, cost) constraints. The experiment was stopped at this point and no data were acquired at other temperatures.

Two examples of the hardware and software inadequacies are the calibration grid and interpolation. The need for the calibration grid is illustrated in Fig. 15, which is a false-color plot of some of the raw data. Each color represents some portion of the scale on the right-hand side of the figure. The scale is proportional to the signal amplitude from the camera; the relationship between them would be calibrated in an actual experiment. If the heat-flux gaugc had been illuminated uniformly and there had been no geometric or optoelectronic distortion in the camera, each triangle would be uniformly the same color. Because that is not the case, a calibration grid must be set up that identifies one region in each triangle as the "correct" amplitude and that normalizes the rest of each triangle, as well as all triangles, to the same amplitude. This lets a uniform static equilibrium temperature distribution be displayed as one temperature, i.e, one color. The calibration grid must be chosen so that each calibration point (one in each triangle) is at the same relative position in each triangle. For efficient data analysis, this process must be automated, but our current software and hardware do not permit it. Instead, each point must be entered by hand, using a mouse in combination with horizontal and vertical cursors that act as cross hairs. Although slow, the process would be acceptable if it were possible to do it only once and use the stored values to overlay on each subsequent image. Unfortunately, this does not work. Subsequent images are slightly different because of the geometric distortion and color-correction effects in the camera and optics. This means the process has to be done for each image, and even then problems exist because of the random variations in the grid points established by the hand entry. Assuming that the calibration grid is, nevertheless, obtained, we run into an even-more-difficult problem. It is necessary to derive a 2-D interpolation map between all pairs of points on the grid and apply this to the data. Although software and hardware exist to do this readily, we do not currently possess it. The software and hardware we have can be made to do most of the tasks, but very slowly, and doing so would require personnel time that we cannot afford.

Pending either our acquiring advanced software and hardware or our discovering another approach that integrates our software and hardware with some that already exists elsewhere, we have brought the data-analysis effort to a logical branch point by creating software with those features that are necessary in either case. The complete in-house-developed program contains far too much material to discuss in detali here. A copy of it is included in Appendix A. Briefly, the user controls the software through a menu-driven program linked to the image-processing library and to custom algorithms developed in-house. All programming is in the MicroSoft C5.1 language.

Once the data are processed (digitized, stored, and operated upon by the grid), the resultant information can be displayed on the RGB monitor superimposed on the heat-flux gauge image. 


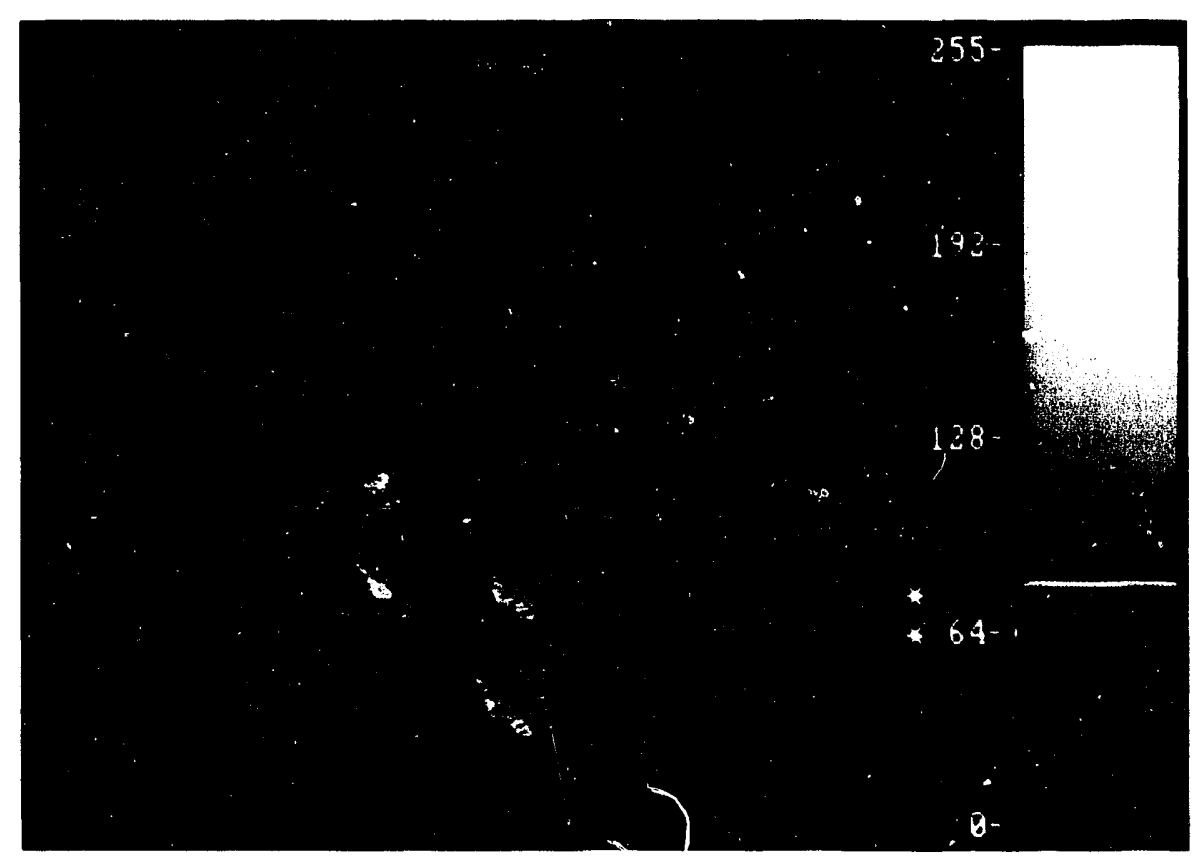

Fig. 15. False-color plot of raw narrow-band-filtered image data for a heat-flux gauge.

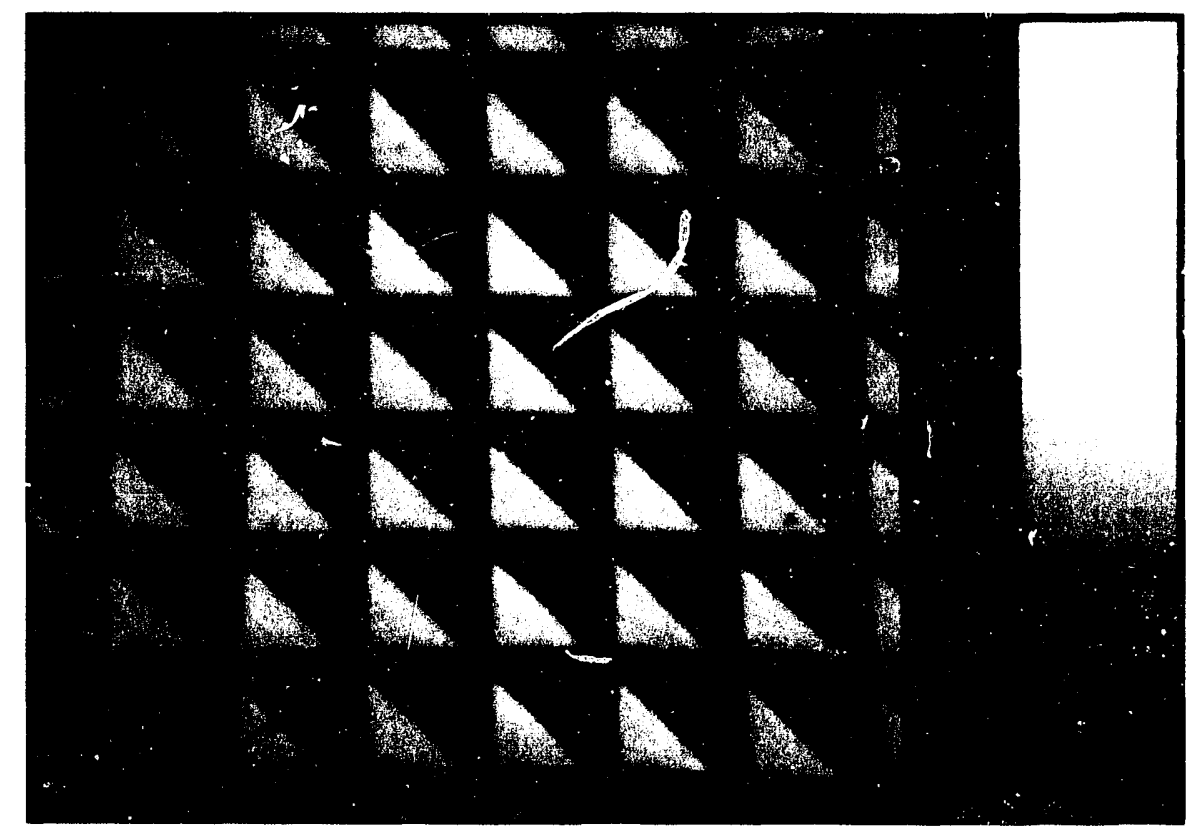

Fig. 16. Display of the video image of a heat-flux gauge.

Figures 16 through 20 are photographs of the processed data as displayed on an RGB monitor. Figure 16 is an image display of an older heat-flux gauge containing $\mathrm{Gd}_{2} \mathrm{O}_{2} \mathrm{~S}$ : Tb on the front surface and $\mathrm{La}_{2} \mathrm{O}_{2} \mathrm{~S}: \mathrm{Eu}$ on the rear surface. Figure 17 is the same image, with a superimposed manually selected grid. The image display can be selected from the menu to show the initial ratio-of-emissionlines data (Fig. 18), the front- and rear-surface calibrated temperature distribution (Fig. 19), and the calculated heat-flux estimates (Fig. 20) (all of these for the grid points). 


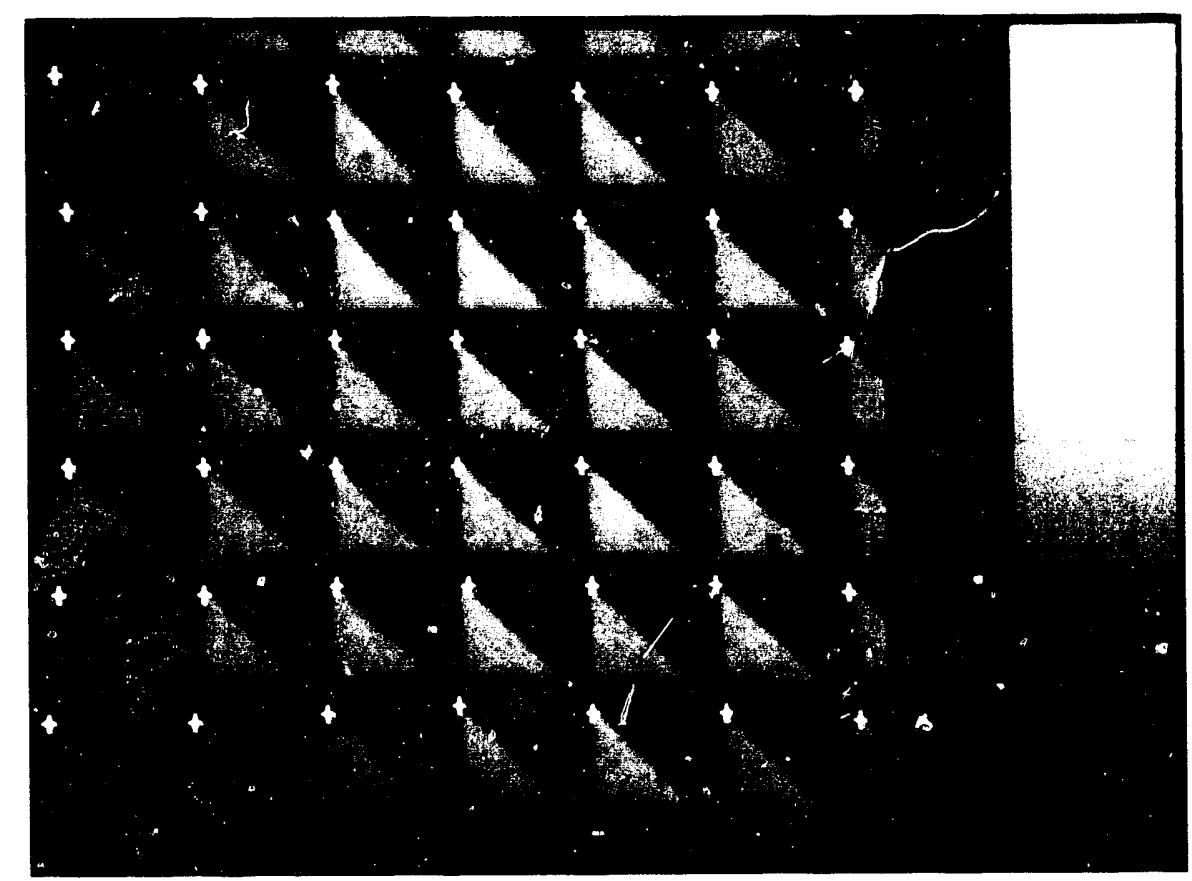

Fig. 17. Heat-flux gauge image with superimposed grid.

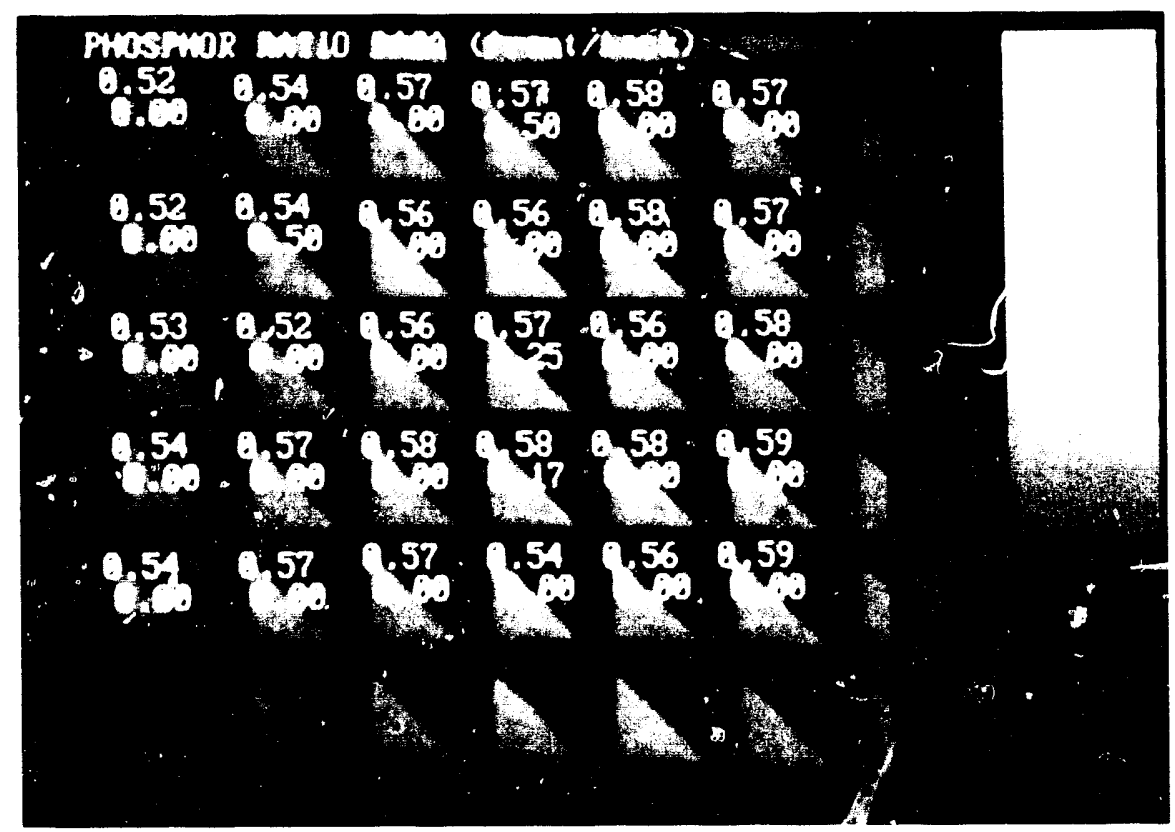

Fig. 18. Image with superimposed ratio-of-emission-lines data. Data pairs are for front and rear surfaces.

A synopsis of the program environment. the calibration parameters, and current data results is saved on the host computer for further analysis by graphics routines or other means. The initial preprocessed images can be maintained on the host computer hard drive. along with the userspecified grid pattern and the gauge calibration data for subsequent reprocessing. This allows maximum flexibility in applying updated hardware. software. and algorithms without having to repeat the experiment. 


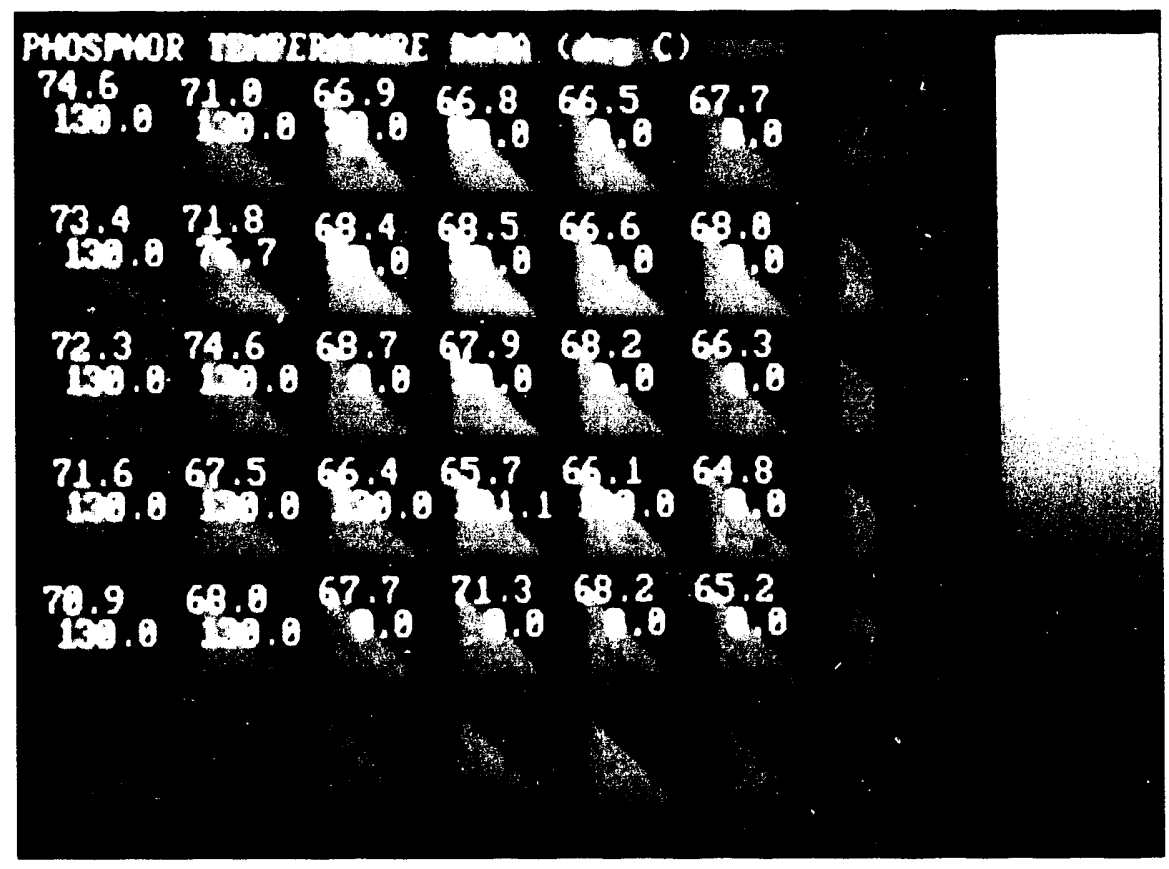

Fig. 19. Image with superimposed temperature data. Pairs of data are for front and rear surfaces.

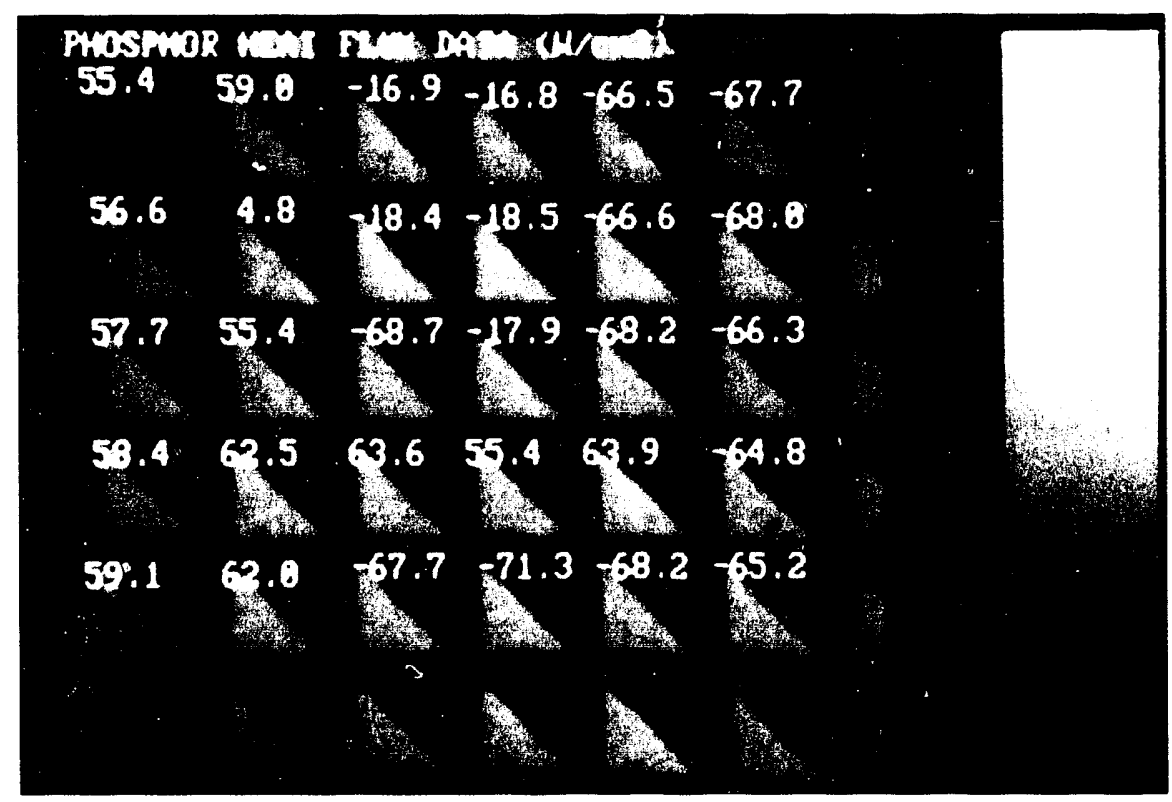

Fig. 20. Image with superimposed heat-flux estimates.

\section{DESIGN OF A FIELD EXPERIMENT}

The sponsor of this project. the Components Branch of the Turbine Engine Division of the Wright Laboratory (WL/POTC), is planning an experiment that models the interior cooling passages in a turbine blade. As part of that experiment at WL, we plan a field test that will be a proof-of-principle demonstration of the 2-D heat-flux gauge. 
In the experiment, the blade interior is a simplified, large-scale mock-up of an actual blade. Besides providing a convenient size in physical dimensions, the scaling permits using lower temperatures, lower hot-gas velocities, etc.

\section{A. Experiment Layout and Parameters}

Figure 21 is a sketch of the experiment, as envisioned by WL. The top view shows heat flow across bars that are covered with our heat-flux gauge. The intent is to measure the detailed heat transfer along the floor of the duct. Air is drawn through the duct by a 750-CFM blower. Flow straighteners are used to quiet the inlet flow. The floor of the duct has 1-in.-square cross-section bars placed from wall to wall at right angles to the flow. The bars, called "turbulators," stir the air flow and increase the heat flux at the floor. At least one bar (probably two) and a section of floor between two bars will be covered with heat-flux gauge. Foil heaters mounted under the gauges provide a constant wall temperature of up to $125^{\circ} \mathrm{F}$. Hieat transfer occurs through the gauge to the air flow in the duct. That part of the top of the duct above the heat-flux gauges is a quartz window for transming the UV light that excites the gauges and the visible light emitted by the gauges. The intensified CID video camera is a type designed by the Neutron Measurements Group (P-15) at Los Alamos. Table II summarizes the expected test conditions.

\section{Large-Scale Cooling-Passage Rig}

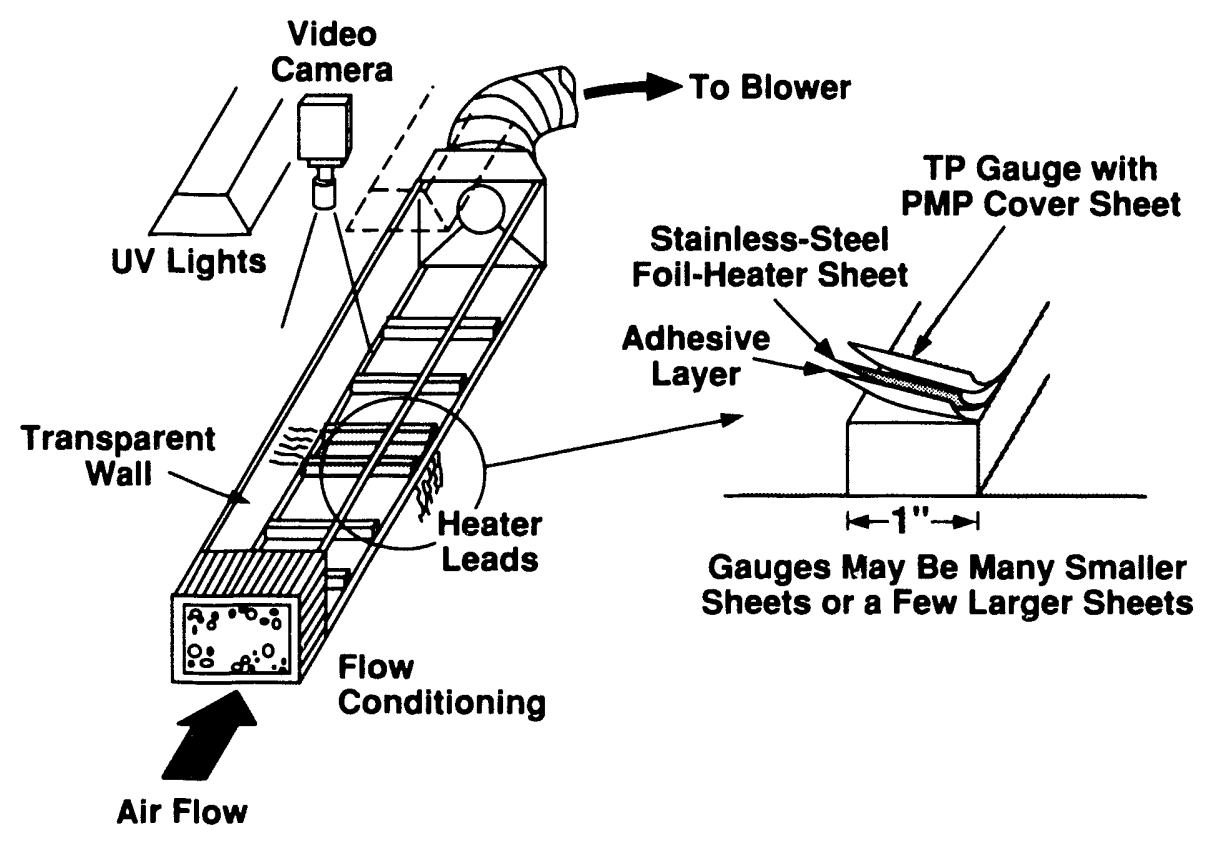

Fig. 21. Experiment layout for the field test.

For this experiment, we decided to make our heat-flux gauges with a single TP. $\mathrm{Gd}_{2} \mathrm{O}_{2} \mathrm{~S}$ : Tb. for several reasons. The data acquisition is simpler; only one camera and two filters are required. This is convenient, because only one camera was available for loan. Filter changes will be by hand, so fewer manual operations are necessary. We have demonstrated that a heat-flux gauge can be made from one TP if the geometry permits identification of the individual elements, as is the case here. Further. we are coding the gauges to eliminate any possibility of ambiguity in that respect. With only two sets of data required per final image, the data manipulation is greatly simplified. 
TABLE II. Test Conditions for Imaging Field Test

Duct length: $5 \mathrm{ft}$

Duct cross section: 6 in. $\times 6$ in.

Centerline velocity $(\max ): 100 \mathrm{ft} / \mathrm{s}$

Reynolds number: $3 \times 10^{5}$

Nusselt number: 2850

Convection coefficient: $85 \mathrm{Btu} / \mathrm{h}-\mathrm{ft}^{2} \cdot{ }^{\circ} \mathrm{F}$

Temperature difference across gauge: $5^{\circ} \mathrm{F}$

Heater temperature $(\max ): 125^{\circ} \mathrm{F}$

Selection of $\mathrm{Gd}_{2} \mathrm{O}_{2} \mathrm{~S}$ : Tb as the only TP in the gauge required limiting the turbulator temperature to $125^{\circ} \mathrm{F}$ (the calibration curve for $\mathrm{Gd}_{2} \mathrm{O}_{2} \mathrm{~S}: \mathrm{Tb}$ flattens out above $125^{\circ} \mathrm{F}$ ), but that turned out to be acceptable. $\mathrm{Gd}_{2} \mathrm{O}_{2} \mathrm{~S}$ :Tb is not as bright as $\mathrm{La}_{2} \mathrm{O}_{2} \mathrm{~S}: \mathrm{Eu}$, but there appear to be ample UV excitation and camera sensitivity available, so this is also acceptable. The gauge-fabrication technique is as described in $\S$ II.

The image-acquisition hardware and software are currently housed in a Compaq-386 portable computer that can easily be transported to the site of the field test.

\section{B. Quantum-Yield Measurement}

Before assessing our likelihood of acquiring usable signals for the field-test experiment, we addressed the question, "How many photons can be delivered to our video camera within a selected bandpass with 'realistic' experimental conditions?" by doing a quantum-yield experiment on a sample of $\mathrm{Gd}_{2} \mathrm{O}_{2} \mathrm{~S}: \mathrm{Tb}$ powder.

The basic concept is simple: excite the TP with UV and measure the fluorescence emission. The experiment layout is shown in Fig. 22. For an excitation source, we selected a fraction of the mercury-arc-lamp emission well-matched to the excitation spectrum of $\mathrm{Gd}_{2} \mathrm{O}_{2} \mathrm{~S}$ : $\mathrm{Tb}$ by using a band-pass filter with peak transmission at $325 \mathrm{~nm}$ and a FWHM of $90 \mathrm{~nm}$. The condenser lens of the lamp focused the output onto a 2 -mm-diam spot size in the plane of the sample. We measured the incident power from the lamp by placing the calibrated radiometer with its detector in the sample plane. Then we moved the radiometer to the position shown in the figure and placed the sample in the sample plane. A fraction of the emission from the sample was focused onto the entrance aperture of the radiometer through a field stop and a band-pass filter that sclected the TP emission line of interest. We considered the solid angle subtended by the receiving lens to determine the fraction of the emitted light seen by the radiometer, as illustrated in Fig. 23. The TP is assumed to emit into $2 \pi \mathrm{sr}$. Let $A_{0}$ be the area of the hemisphere of radius $r$ through which the emitted radiant flux passes; let $A_{l}$ be the area of the lens; and let $I_{0}$ and $I$ be the total TP emission into $2 \pi$ and the emission measured by the radiometer, respectively. Assuming a Lambertian source, we have

$$
\frac{A_{0}}{A_{l}} I=I_{0} \cos \theta .
$$




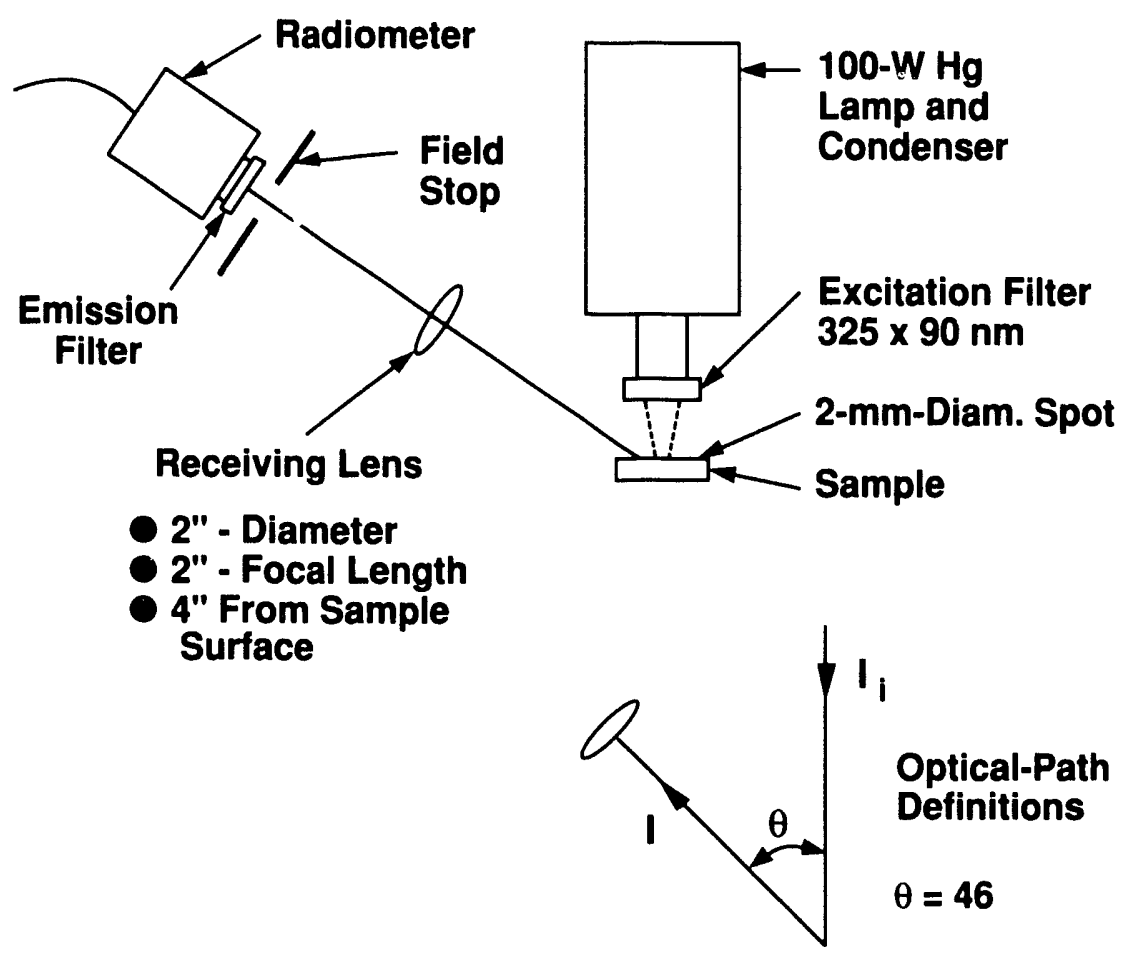

Fig. 22. Experiment layout for quantum-yield measurement.

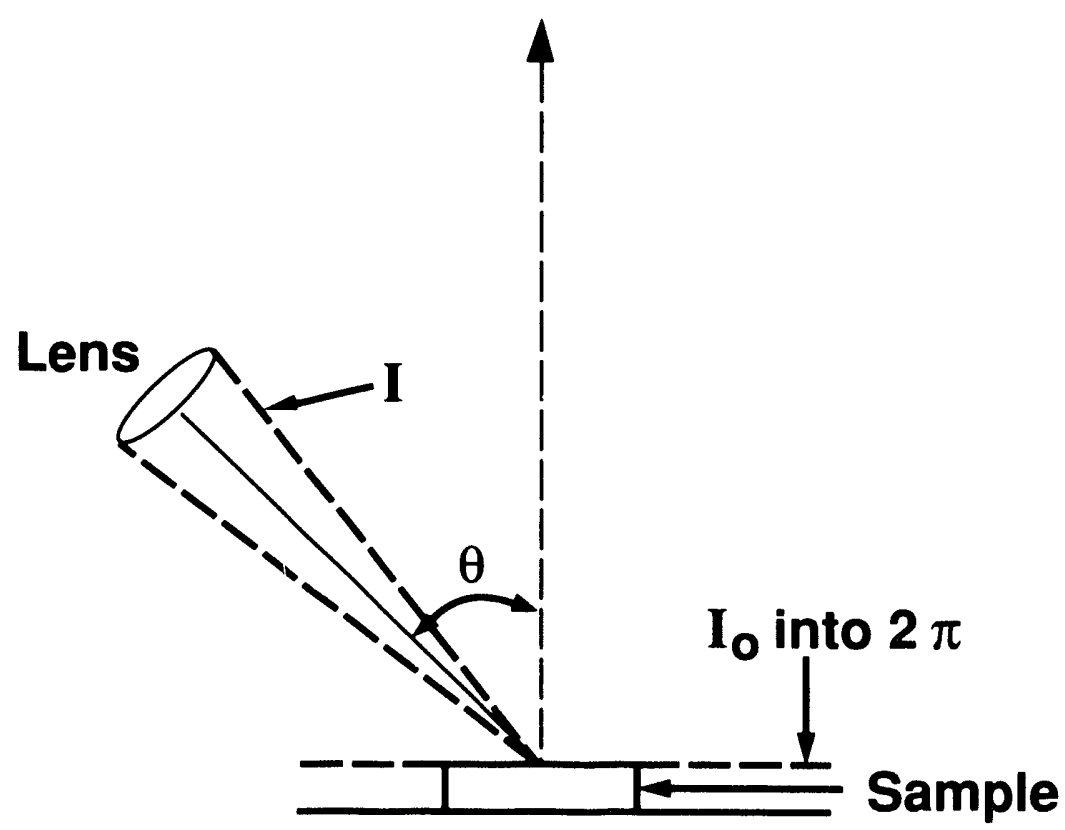

Fig. 23. Solid-angle definitions. 
TABLE III. Quantum Yield of $\mathrm{Gd}_{2} \mathrm{O}_{2} \mathrm{~S}: \mathrm{Tb}$

Small Excitation Spot

$I_{i}=2.38 \times 10^{-2} \mathrm{~W}$

\begin{tabular}{|c|c|c|c|c|c|c|}
\hline $\begin{array}{c}\text { Emission } \\
\text { Filter }\end{array}$ & $\begin{array}{c}\text { Radiometer } \\
\text { Output, } \\
\text { A }\end{array}$ & $\begin{array}{c}\text { Radiometer } \\
\text { Conversion, } \\
\text { A/W }\end{array}$ & $\begin{array}{c}I, \\
\text { Phosphor } \\
\text { Power into } \\
\text { Radiometer, } \\
\text { W }\end{array}$ & $\begin{array}{c}I_{0}, \\
\text { Total } \\
\text { Phosphor } \\
\text { Power, } \\
\text { W }\end{array}$ & $\begin{array}{c}\text { Filter } \\
\text { Correction }\end{array}$ & $\begin{array}{c}\Phi, \\
\text { Quantum } \\
\text { Yield }\end{array}$ \\
\hline $422 \times 35$ & $177 \times 10^{-9}$ & 0.129 & $1.37 \times 10^{-6}$ & $6.31 \times 10^{-5}$ & 5.16 & 0.014 \\
\hline $540 \times 10$ & $2138 \times 10^{-9}$ & 0.250 & $8.55 \times 10^{-6}$ & $3.93 \times 10^{-4}$ & 2.62 & 0.043 \\
\hline $543.8 \times 3$ & $2080 \times 10^{-9}$ & 0.250 & $8.32 \times 10^{-6}$ & $3.83 \times 10^{-4}$ & 3.56 & 0.057 \\
\hline
\end{tabular}

Substituting the area of the lens, $\pi(2.54 \mathrm{~cm})^{2}=20.3 \mathrm{~cm}^{2}$, the area of the hemisphere, $(1 / 2) 4 \pi r^{2}=$ $649 \mathrm{~cm}^{2}$, and the cosine of $46 \circ$, we find that $I_{0}=46.0 I$; that is, about $1 / 46$ of the total emitted fluorescence is intercepted by the radiometer.

We did the experiment with three different band-pass filters at the radiometer: one $35 \mathrm{~nm}$ wide centered at $422 \mathrm{~nm}$; one $10 \mathrm{~nm}$ wide centered at $540 \mathrm{~nm}$; and one $3 \mathrm{~nm}$ wide centered at $543.8 \mathrm{~nm}$. The $422-\mathrm{nm}$ filter is the closest available to the important terbium thermographic-emission line at $415 \mathrm{~nm}$. The 540-nm and 543.8-nm filters were chosen to look at the bright 541-nm terbiumemission line that we use for a reference. Table III summarizes the results of the first part of the experiment. Several items in the table may need further explanation. The radiometer output is the measured TP signal, with the given lamp, TP, experiment geometry, and band-pass filter. The radiometer conversion corrects for the responsivity of the radiometer's silicon detector, which varies as a function of wavelength. The TP intensity, $I$, has been defined earlier, as has $I_{0}$. The filter correction uses the band-pass curves for the optical filters to correct for their transmission at the TP's emission wavelength. The incident excitation power, $I_{i}$, was $2.38 \times 10^{-2} \mathrm{~W}$ and $1.72 \times 10^{-4} \mathrm{~W}$ for data reported in Tables III and IV, respectively. The radiometer's detector surface area is one square centimeter, and one-to-one imaging optics were used to transfer the TP image to the detector surface. Consequently, for simplicity, all intensities are reported in watts. The last column is the desired result, the quantum yield, or ratio of incident UV intensity to total fluorescence output, $\Phi=\left(I_{0} / I_{i}\right) \times($ filter correction $)$.

In the second part, we enlarged the incident UV spot to cover a larger area more analogous to the actual situation in a field experiment. The beam diameter for this part was $16.7 \mathrm{~cm}$ (about $6 \mathrm{in}$.). The angle between the center of the incident beam and the optical axis of the radiometer was 330 . We list the results in Table IV. Of most importance is the observation that the change in quantum yield is negligible. This is gratifying, because it suggests that we can use a variety of experimental geometries with confidence that the quantum yield will remain relatively constant. All we need is $I_{i}$ and the solid angle subtended by the imaging optics.

\section{Measurement of Video-Camera Sensitivity}

Once we determined the quantum yield of the TP, it became possible to estimate, from the specifications, whether a given video camera could be used in the field test. For example, we 
TABLE IV. Quantum Yield of $\mathrm{Gd}_{2} \mathrm{O}_{2} \mathrm{~S}: \mathrm{Tb}$

Large Excitation Spot

$I_{i}=1.72 \times 10^{-4} \mathrm{~W}$

\begin{tabular}{ccccccc} 
Emission & $\begin{array}{c}\text { Radiometer } \\
\text { Output, } \\
\text { Filter }\end{array}$ & $\begin{array}{c}\text { Radiometer } \\
\text { Conversion, } \\
\mathrm{A} / \mathrm{W}\end{array}$ & $\begin{array}{c}\mathrm{C}_{0}, \\
\text { Power into } \\
\text { Radiometer, } \\
\mathrm{W}\end{array}$ & $\begin{array}{c}\text { Total } \\
\text { Phosphor } \\
\text { Power, } \\
\mathrm{W}\end{array}$ & $\begin{array}{c}\text { Filter } \\
\text { Correction }\end{array}$ & $\begin{array}{c}\Phi, \\
\text { Quantum } \\
\text { Yield }\end{array}$ \\
\hline & & & & & & \\
$422 \times 35$ & $1.4 \times 10^{-9}$ & 0.129 & $1.08 \times 10^{-8}$ & $4.13 \times 10^{-7}$ & 5.16 & 0.012 \\
$540 \times 10$ & $16.5 \times 10^{-9}$ & 0.250 & $6.60 \times 10^{-8}$ & $2.51 \times 10^{-6}$ & 2.62 & 0.038 \\
$543.8 \times 3$ & $13.6 \times 10^{-9}$ & 0.250 & $5.44 \times 10^{-8}$ & $2.07 \times 10^{-6}$ & 3.56 & 0.043 \\
\hline \hline
\end{tabular}

expect the irradiance caused by the fluorescence in the field test (using an identical UV source) to be in the range $10^{-8}$ to $10^{-9} \mathrm{~W} / \mathrm{cm}^{2}$. One video camera we considered was the Xybion SPL- 4031 . According to the specifications, this camera's peak sensitivity is typically better than $10^{-5} \mathrm{fc}$, which is approximately $1.6 \times 10^{-11} \mathrm{~W} / \mathrm{cm}^{2}$. This would give it a signal-to-noise ratio of about 100 , so we might expect it to be satisfactory under good experimental conditions.

We were able to borrow an intensified CID camera from the Neutron Measurements Group (P-15) at Los Alamos to measure the video signal produced by a TP target and to confirm our predictions. We set it up in the experiment diagrammed in Fig. 24. We used the same mercury-arc lanp and $325 \times 90$ excitation filter used in the previous experiment and the same $422 \times 35$ filter to select the dim but crucial-- 415-nm TP emission line. The TP sample was made by air-brushing a $10 \times 10$ matrix of triangles of $\mathrm{Gd}_{2} \mathrm{O}_{2} \mathrm{~S}: \mathrm{Tb}$ onto a 1-in.-square quartz flat.

Figure 25 shows oscilloscope traces of typical video signals obtained. They show one line of video across the TP matrix. To obtain Fig. 25a, we removed the band-pass filter, which gave a very large signal that could saturate the camera. We also turned down the intensifier gain to minimum. The result shows that the camera saturates at about $500 \mathrm{mV}$ output. We installed the band-pass filter and acquired Fig 25b, which shows that the signal from the 415-nm line nearly saturates the camera with the intensifier gain set at maximum. This result demonstrates that we have enough sensitivity to use the full dynamic range of the camera for room-temperature signals. We simulated weak signals by turning down the intensifier gain and acquired Fig. 25c, which shows the noise level. Clearly, we will be able to resolve a $10-\mathrm{mV}$ signal. This result, when combined with the intensifier's variable gain, confirms a very large dynamic range and a probable excellent signal-to-noise ratio in the field test using an identical camera.

Note that the cardboard fixture for the filter was only 1-in. square and therefore subtended a smaller area than the lens in the quantum-yield experiment. We would get a $5 \times$ increase with the right-sized filter and another factor of 3 with the right wavelength filter, for a total increase of 15 times as many photons per unit area compared with this experiment. 


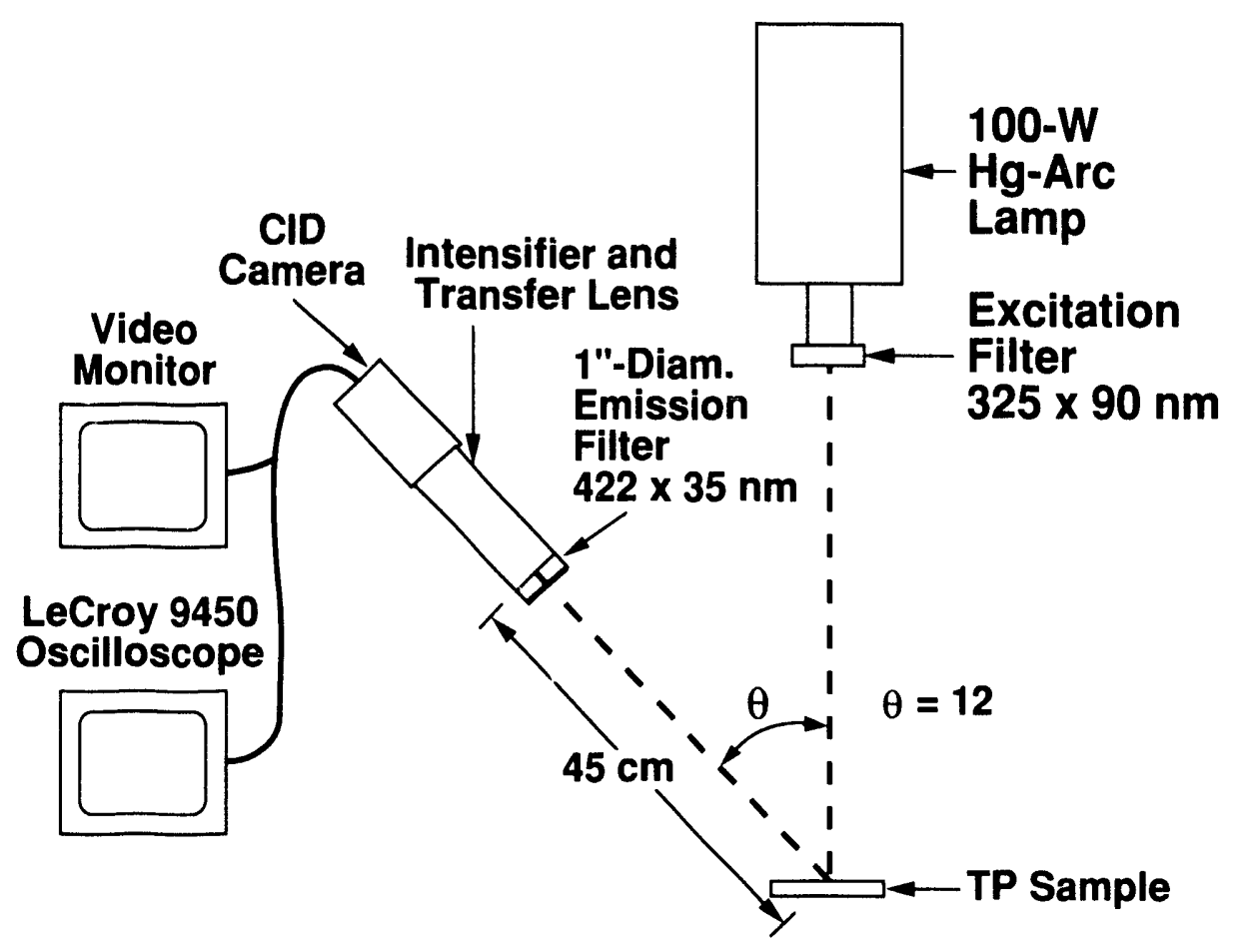

Fig. 24. Experiment layout to test video-camera sensitivity.

\section{PATENTS}

We were recently notified that the first patent has been issued for the heat-flux gauge, and two more are pending. A disclosure for a fourth patent, an insulatorless high-temperature version of the heat-flux gauge, was recently filed by one of the authors (BWN).

\section{ACKNOWLEDGEMENTS}

Many people assisted us in the various tasks described here. We particularly wish to recognize the contributions of Gary Grier and Wil Lewis at EG\&G; Mike Barron and Ralph Stiglich at Los Alamos for helping to specify cameras; George Yates and Steve Jaramillo at Los Alamos for lending us the P-15 video camera; Mike Cates at Oak Ridge for his helpful comments; Lee Zevenberger at Oak Ridge for electron-beam depositions on samples; John Jordan at Oak Ridge for extensive software-engineering support; Bill Stange at WL for many miscellaneous bits of help; Charlie MacArthur at WL for much input, many useful discussions, and a great deal of patience; and to WL for sponsoring the project.

\section{REFERENCES}

1. W. D. Turley et al., "The Design and Characterization of a Prototype Cptical Heat-Flux Gauge," Los Alamos National Laboratory report LA-11408-MS (January 1989).

2. R. K. Williams, R. S. Graves, and J. P. Moore, "A Study of the Effects of Several Variables on the Thermal Conductivity of $2 \frac{1}{4}$ Cr-1 Mo Steel," Oak Ridge National Laboratory report ORNL 5313 (April 1978). 


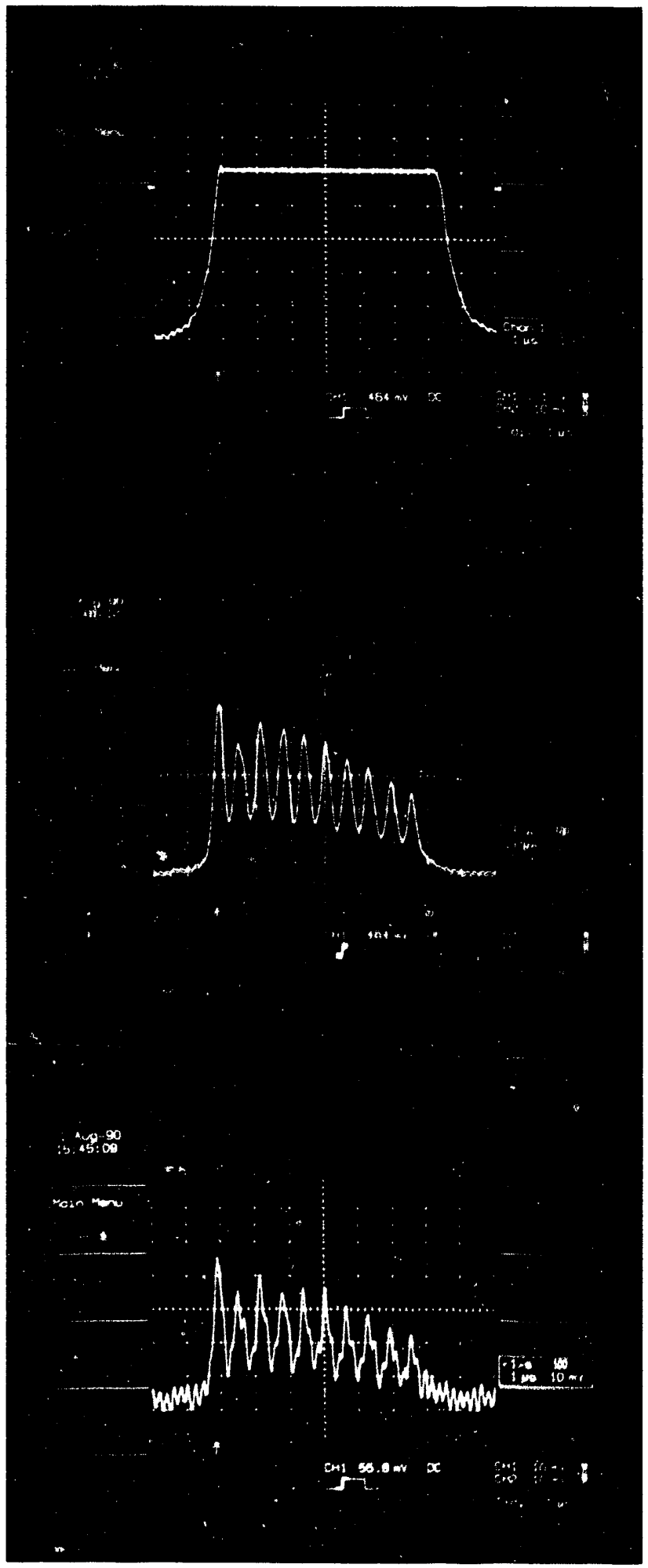

(a)

(b)

(c)

Fig. 25. Results of video-camera experiment: a (top) saturation; b (middle) signal from 415-nm line: c (bottom) small signal. showing noise level. 
3. R. K. Williams, R. K. Nanstad, R. S. Graves, and R. G. Berggren, "Irradiation Effects on Thermal Conductivity of a Light-Water Reactor Pressure Vessel Steel," J. Nucl. Mater. 115, 211-215 (1983).

4. J. P. Moore, T. G. Kollie, R. S. Graves, and D. L. McElroy, "Thermal Conductivity Measurements on Solids Between 20 and $150^{\circ} \mathrm{C}$ Using a Comparative-Longitudinal Apparatus: Results on $\mathrm{MgO}, \mathrm{BeO}, \mathrm{ThO}_{2}, \mathrm{Th}_{x} \mathrm{U}_{1-x} \mathrm{O}_{2+y}$ and $\mathrm{Al}-\mathrm{UO}_{2}$ Cermets," Oak Ridge National Laboratory Report ORNL 4121 (June 1967).

5. M. J. Laubitz, T. Matsumura, and P. J. Kelly, "Transport Properties of the Ferromagnetic Metals. II Nickel," Can. J. Phys. 54, 92-102 (1976).

6. M. Barisoni, R. K. Williams, and D. L. McElroy, "Physical Properties of Indium from 77 to $350 \mathrm{~K}$," Thermal Conductivity, Proceedings of the Seventh Conference, National Bureau of Standards Special Publication 302, pp. 279-292 (September 1968).

7. R. Berman, Thermal Conduction in Solids, p. 104ff (Clarendon: Oxford University Press, 1976).

8. Y. S. Touloukian, R. W. Powell, C. Y. Ho, and P. G. Klemens, Thermophysical Properties of Matter, vol. 2: Thermal Conductivity - Nonmetallic Solids," p. 240 (New York and Washington: IFI/Plenum, 1970).

9. A. H. Epstein, G. R. Guenette, and R. G. Norton, "High-Frequency Response Heat-Flux Gauge," Rev. Sci. Instrum. 57, 639-649 (1986).

10. B. K. P. Horn, Robot Vision, p. 206 (New York: McGraw-Hill, 1986).

11. W. H. Fonger and C. W. Struck, "Eu ${ }^{+++}{ }^{5} \mathrm{D}$ Resonance Quenching to the Charge-Transfer States in $\mathrm{Y}_{2} \mathrm{O}_{2} \mathrm{~S}, \mathrm{La}_{2} \mathrm{O}_{2} \mathrm{~S}$, and LaOCl," J. Chem. Phys. 52(12), 6364-6372 (1970).

12. B. W. Noel, W. D. Turley, M. R. Cates, and K. W. Tobin, "Two-Dimensional Temperature Mapping Using Thermographic Phosphors," presented at the High Temperature Sensors Symposium, $177^{\text {th }}$ Meeting of the Electrochemical Society, Montréal, Quebec, Canada, Los Alamos National Laboratory report LA-UR-90-1534 (May 1990).

\section{APPENDIX A}

This appendix contains a listing of the C-based program that we created for processing image data from the TP 2-D heat-flux gauge.

HEAT.H, p. 34, is the header control file for the executable heat-flux gauge routine HEAT.EXE.

HEAT.C, p. 39, is the main control routine. It tests for the proper configuration of the hardware, and then calls the menu-control routine.

HEATMENU.C, p. 40, is the main menu module. All submenu and function calls are made from it. 
HEATMISC.C, p. 47, contains miscellaneous functions that initialize and end program functions, display messages, control cursors, etc. These functions can be called by many of the program modules.

HEATFILE.C, p. 53, contains functions for disk-file operations, such as storing and retrieving data.

HEATSLCT.C, p. 60, controls the capture and display of video data. Data captured to the appropriate image buffers from an RS-179 video source can be selected from the menu by the user for display on an RGB interlaced monitor.

HEATPROC.C, p. 63, contains process routines that evaluate data buffers to estimate 2-D temperature and heat-flux values.

HEATCOLR.C, p. 76, is a collection of utilities that produce false-color displays. Zoom, pan, scroll, etc., are included.

HEATCONT.C, p. 80, contains functions that modify and control the look-up tables.

An example result file begins on p. 82 and a compiler/library list is on p. 84 .

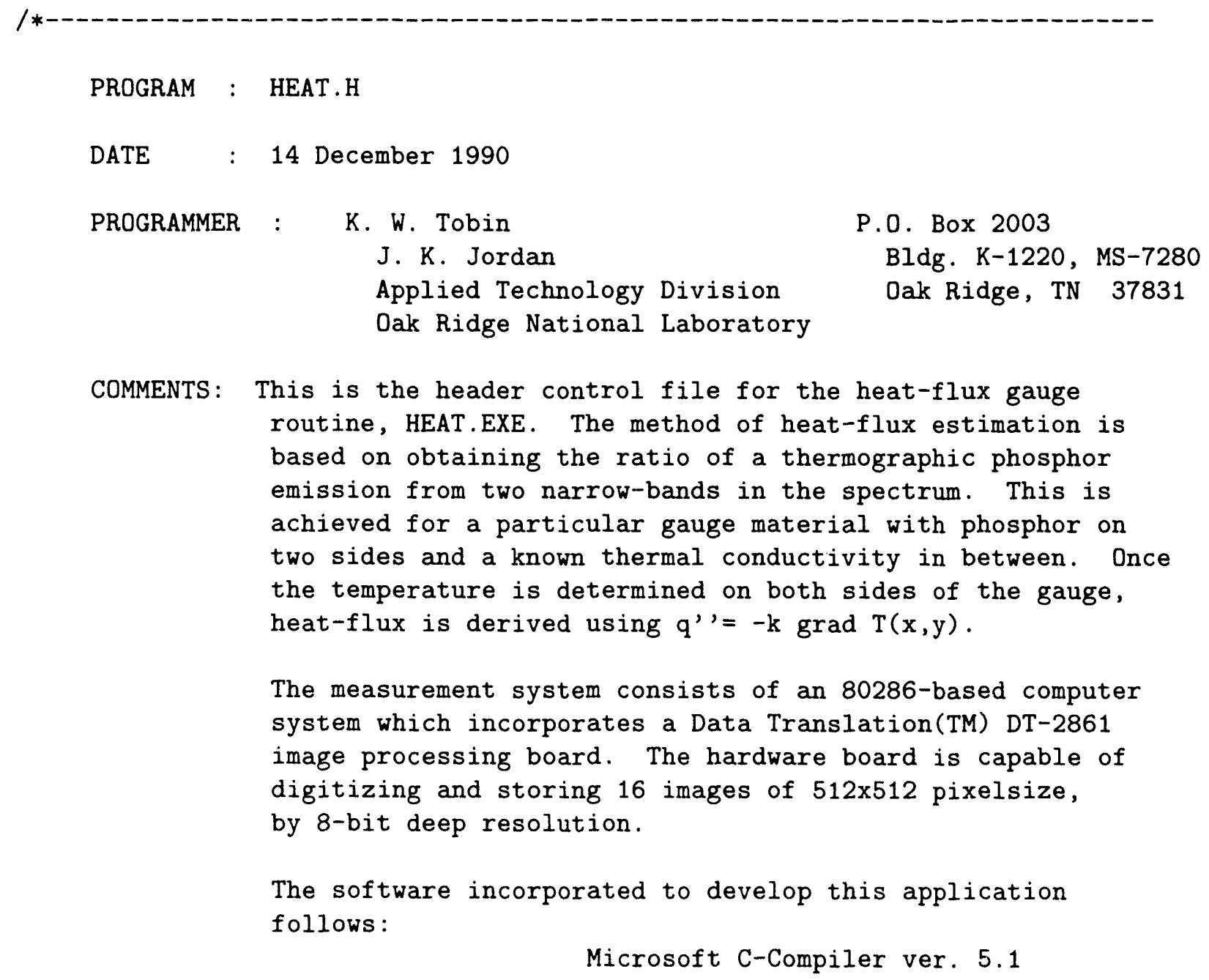


Vitamin-C Menu Software ver. 3.1a

DT-IRIS SP0215 for the DT-2861

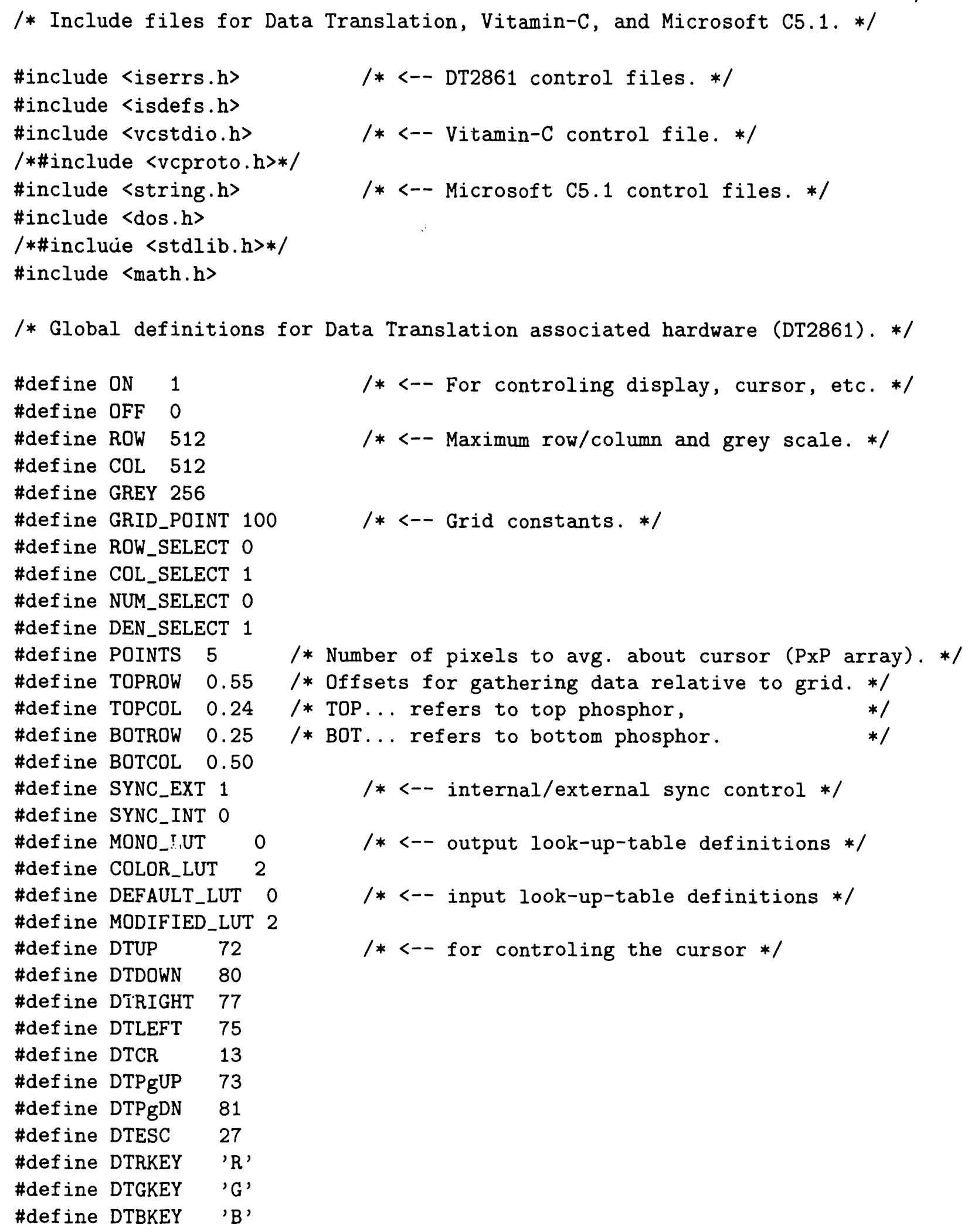


\#define TOTAL_IMAGES 7

\#define DATA_IMAGES 4

\#define NUMERATOR

\#define NUMERATOR_BACK

\#define DENOMINATOR

\#define DENOMINATOR_BACK 3

\#define RATIO

\#define TEMPERATURE 5

\#define HEAT_FLUX 6

\#define NUMERATOR_CORR 7

\#define DENOMINATOR_CORR 8

\#define GRAPHIC_BUF 9
/* <-- number of defined data buffers */

/* <-- number of data images $* /$

/*<-- image buffers */

/* Global definitions for DOS system */

\#def ine SUCCESS 0

/* <-- Subroutine test parameter. */

\#define FAILURE 1

\#define PATHSTRLEN 28

\#define FILENAMLEN

\#define TITLE

9

\#define PATH

"HEAT-FLUX GAUGE - Ver. 1.0 (ORNL/ATD)"

\#define NONE_IMAGE "heat.img"

\#define STARTFILE "no file"

/* Base units for current configuration */

*tefine TUNIT " $\mathrm{xC}$ "

\#define DTTUNIT

\#desine XUNIT

"deg C"

"cm"

/*<-- temperature */

\#define KUNIT

"W/cmxC"

/* (for DT2861 display) */

\#define DTKUNIT

\#define QUNIT

"W/cm C"

"W/cm\}"

/* <-- distance

/* <-- thermal conductivity $* /$

\#define DTQUNIT

"W/cm2"

/* (for DT2861 display) */

/* <-- heat flux */

/* (for DT2861 display) */

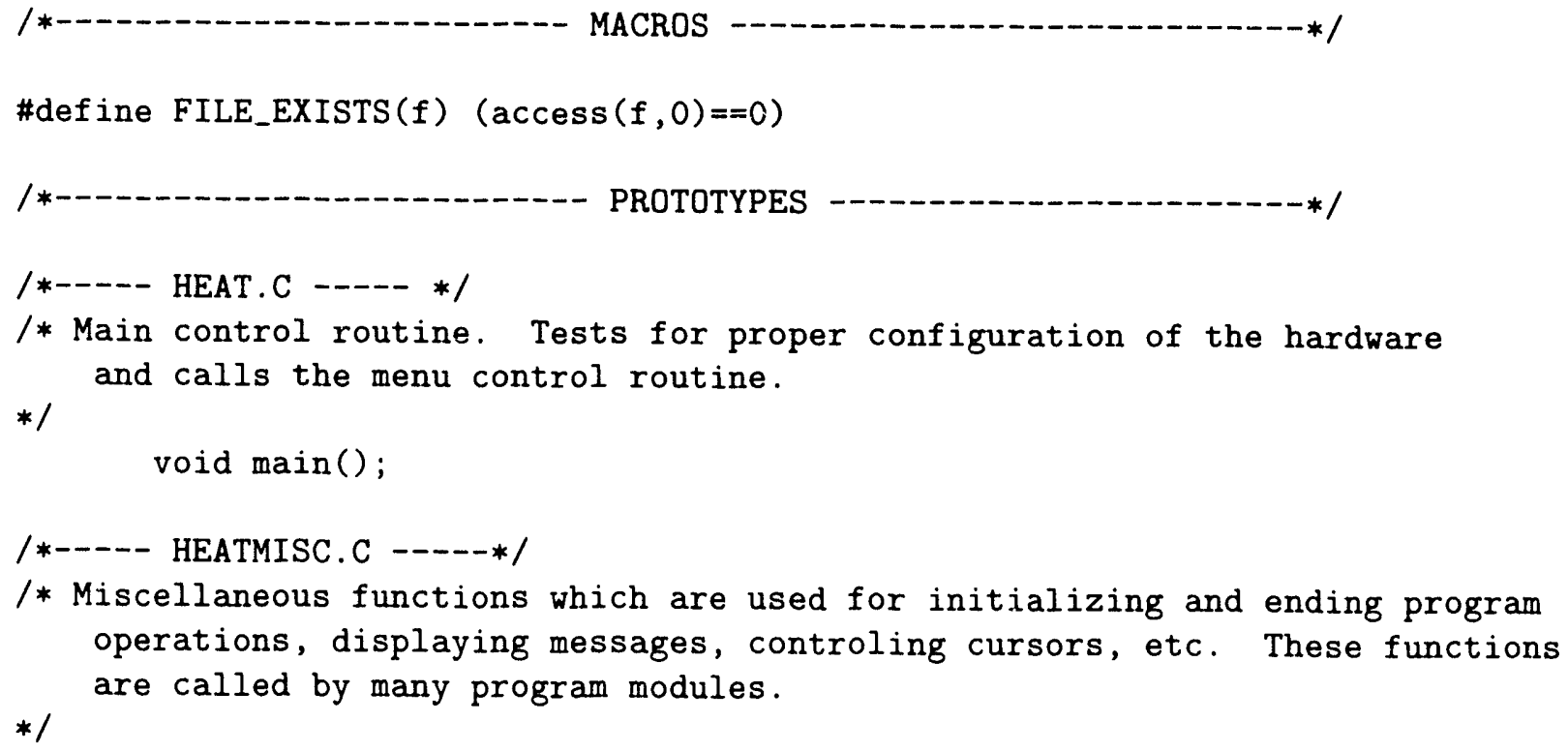




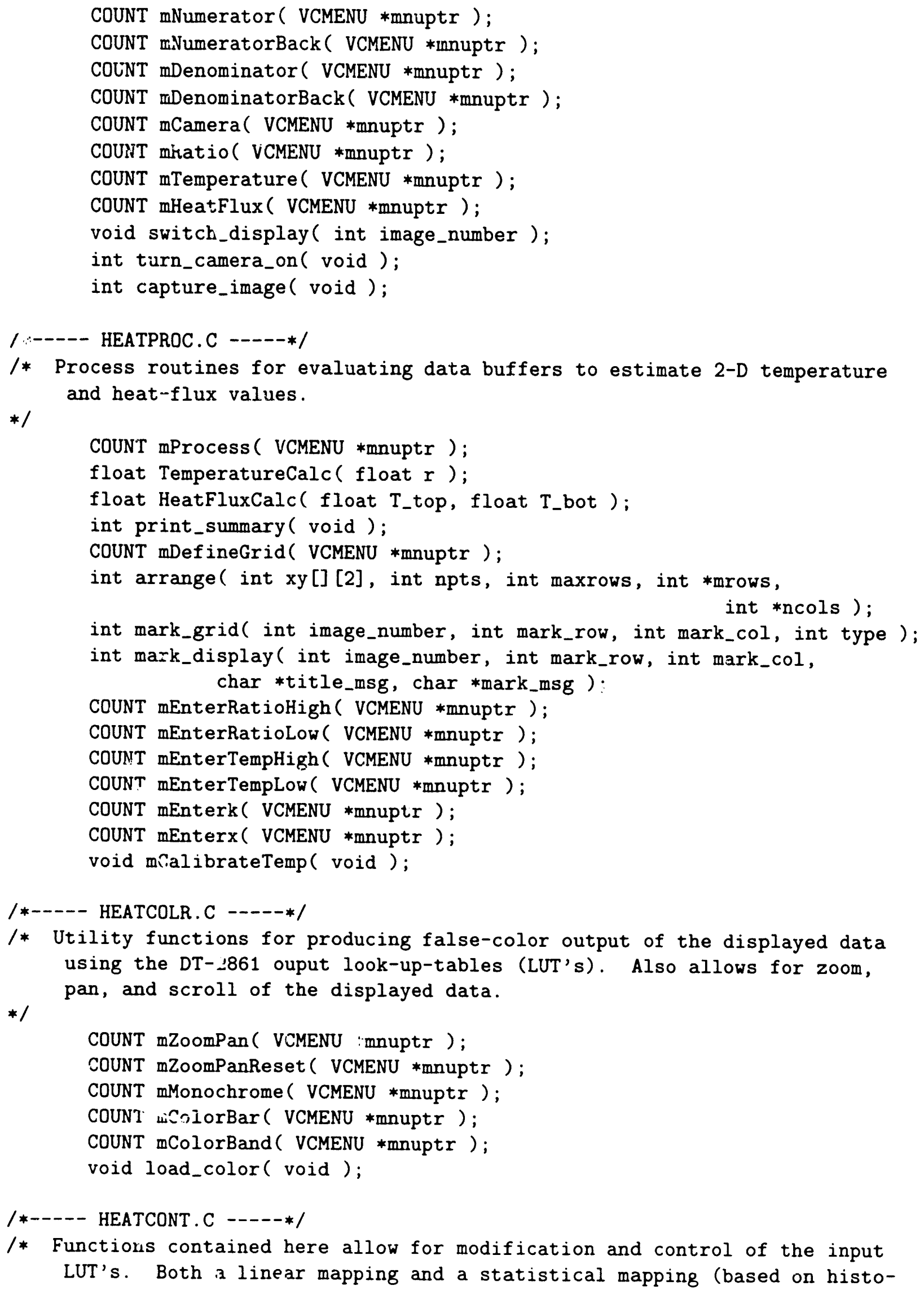

/* Functions contained here allow for modification and control of the input LUT's. Both a linear mapping and a statistical mapping (based on histo- 
gra.i equalization) are included. Once these mappings are defined they can be enablid or disabled at any time.

*/
COUNT mContrastLinear ( VCMENU *mnuptr );
COUNT mContrastEqual ( VCMENU *mnuptr);
COUNT mSelectModLUT ( VCMENU *mnuptr );
COUNT mSelectDefLUT ( VCMENU *mnuptr);

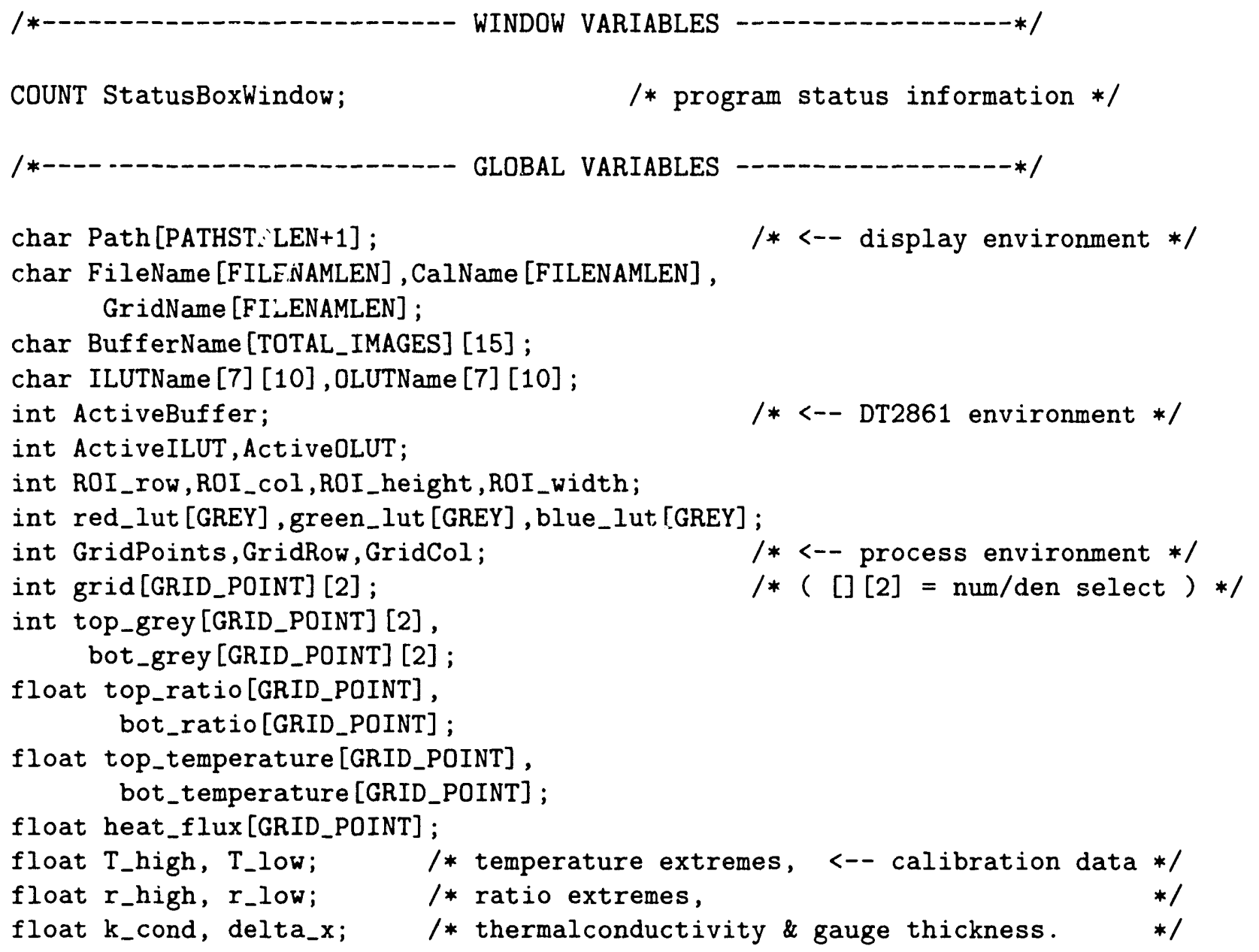




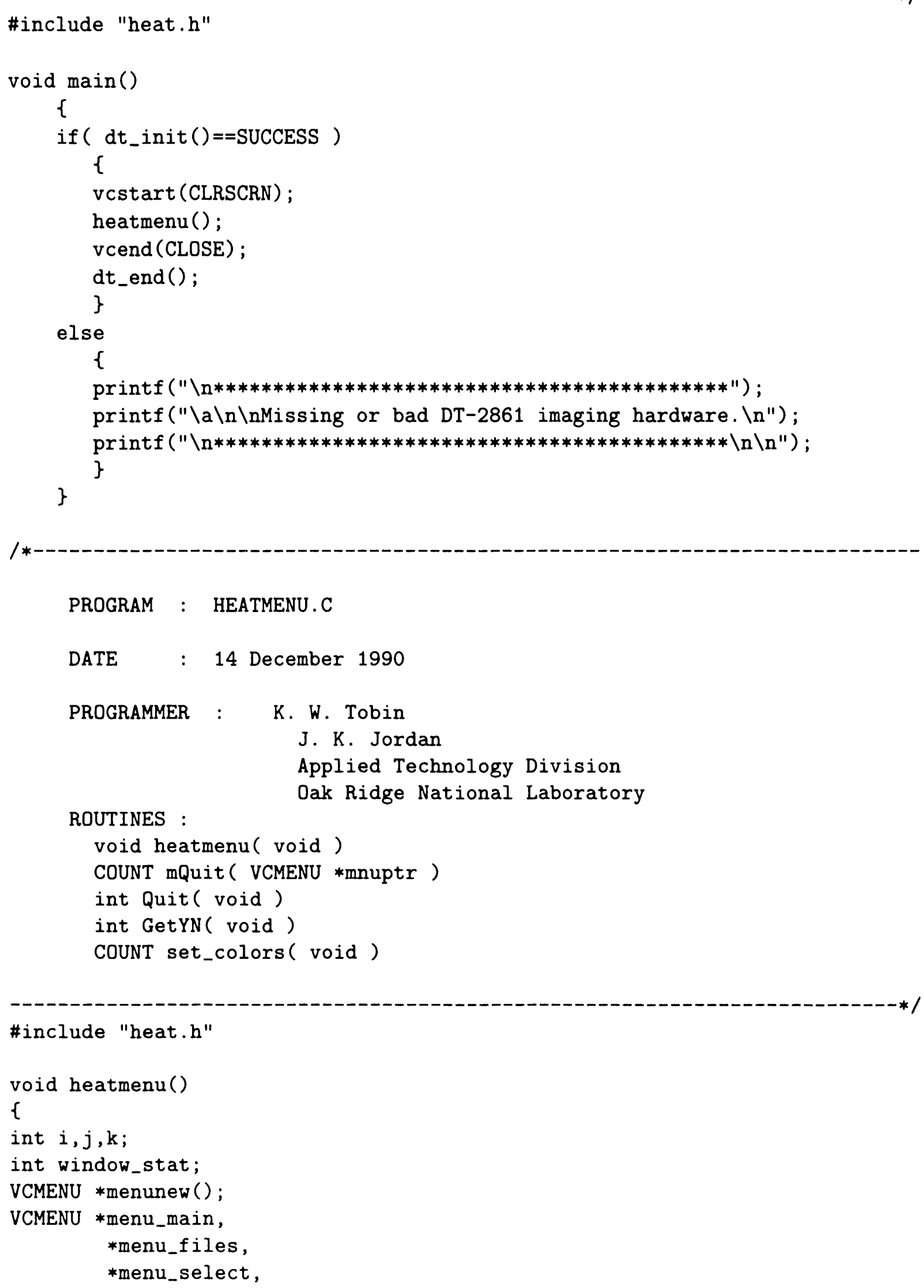


"Exit and return to DOS.", "NO HELP", ESCAPE);

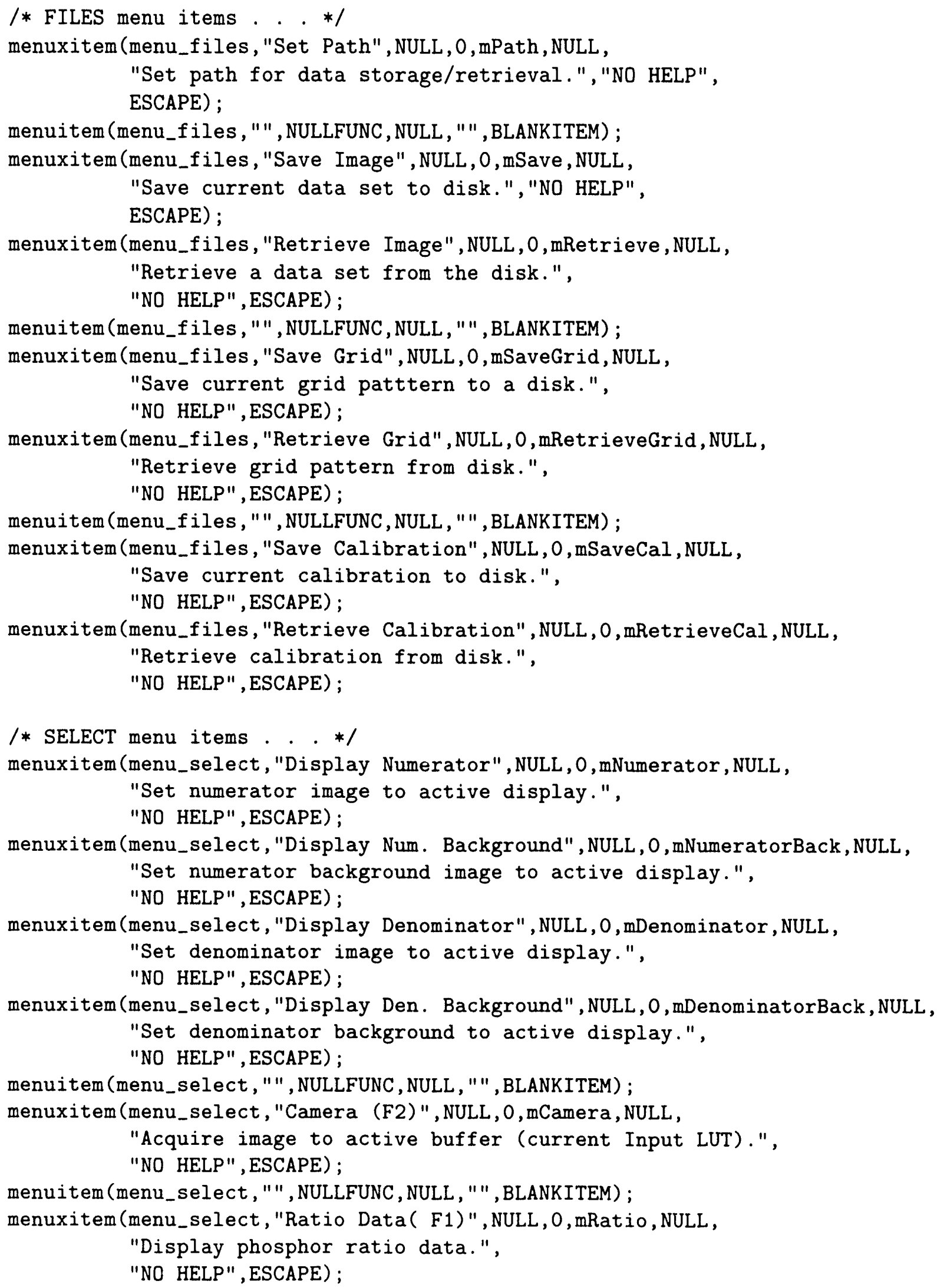




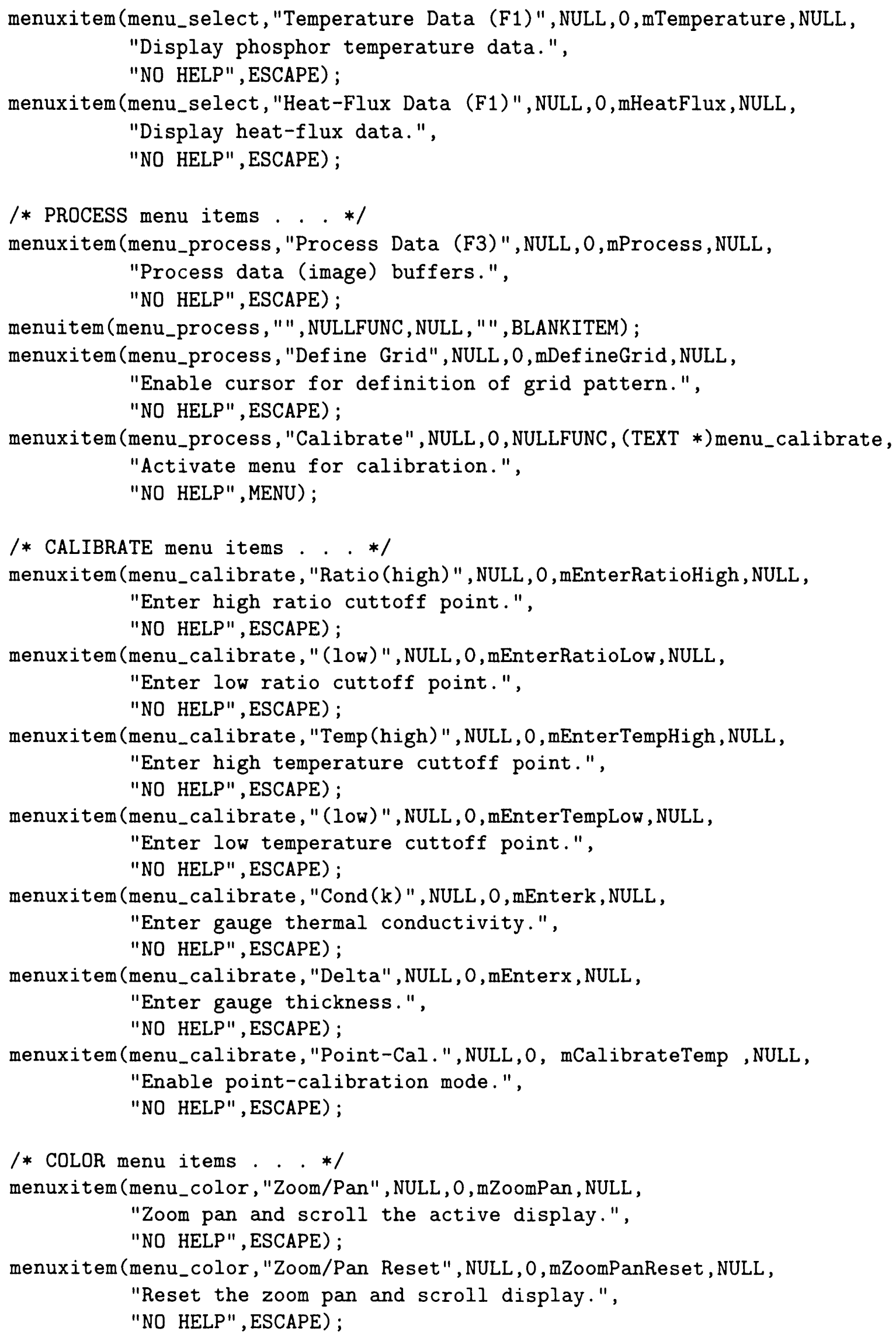




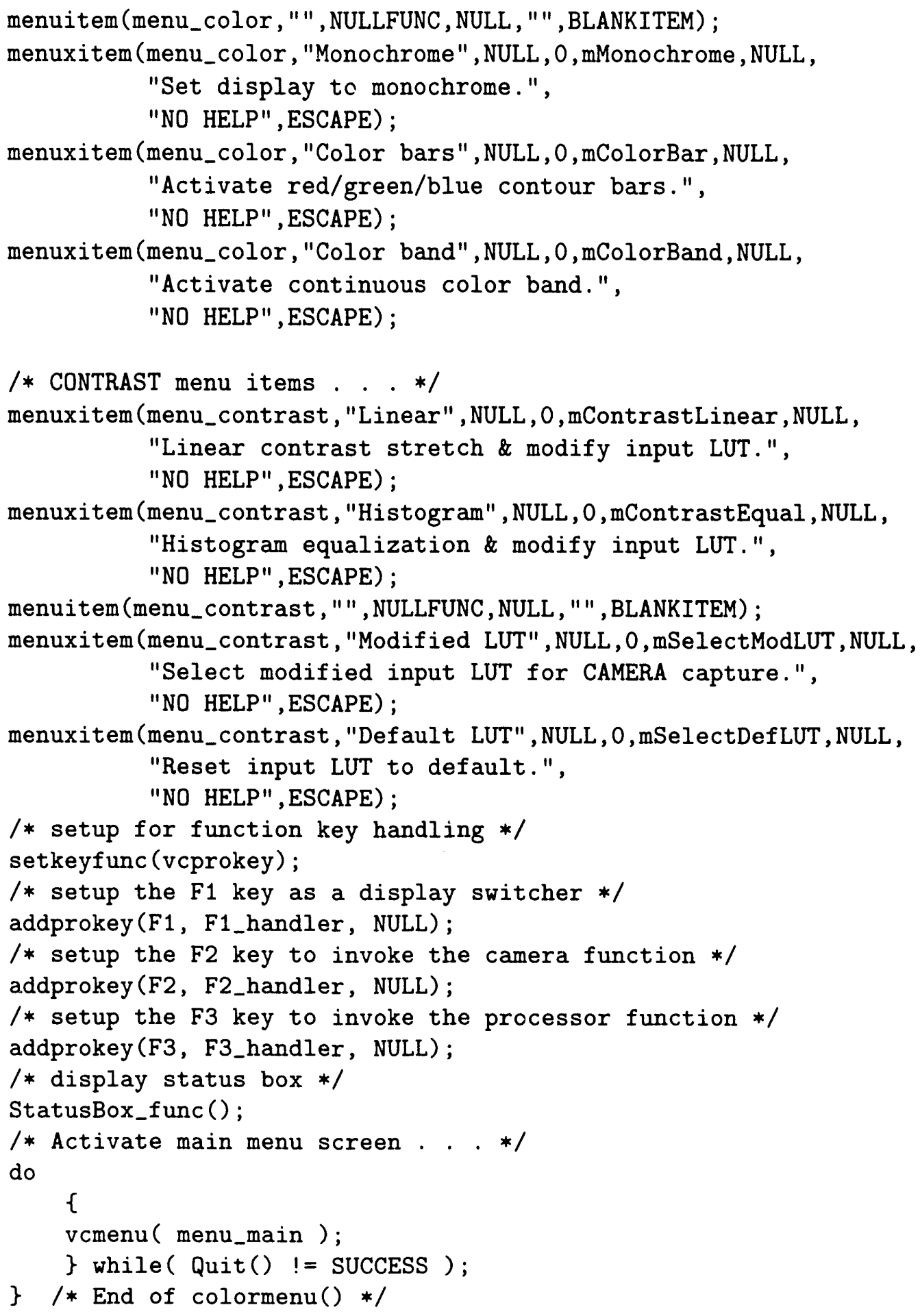




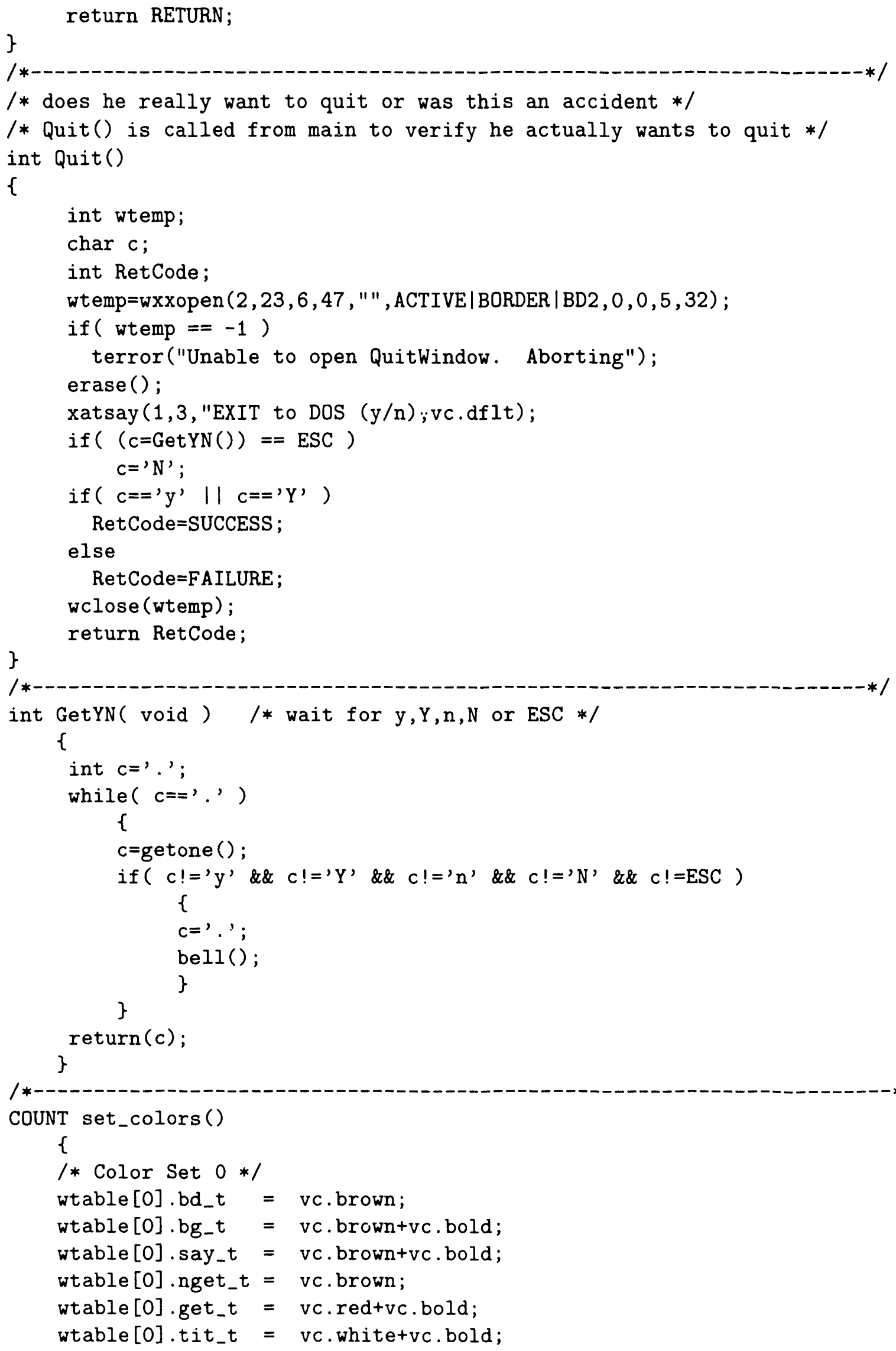




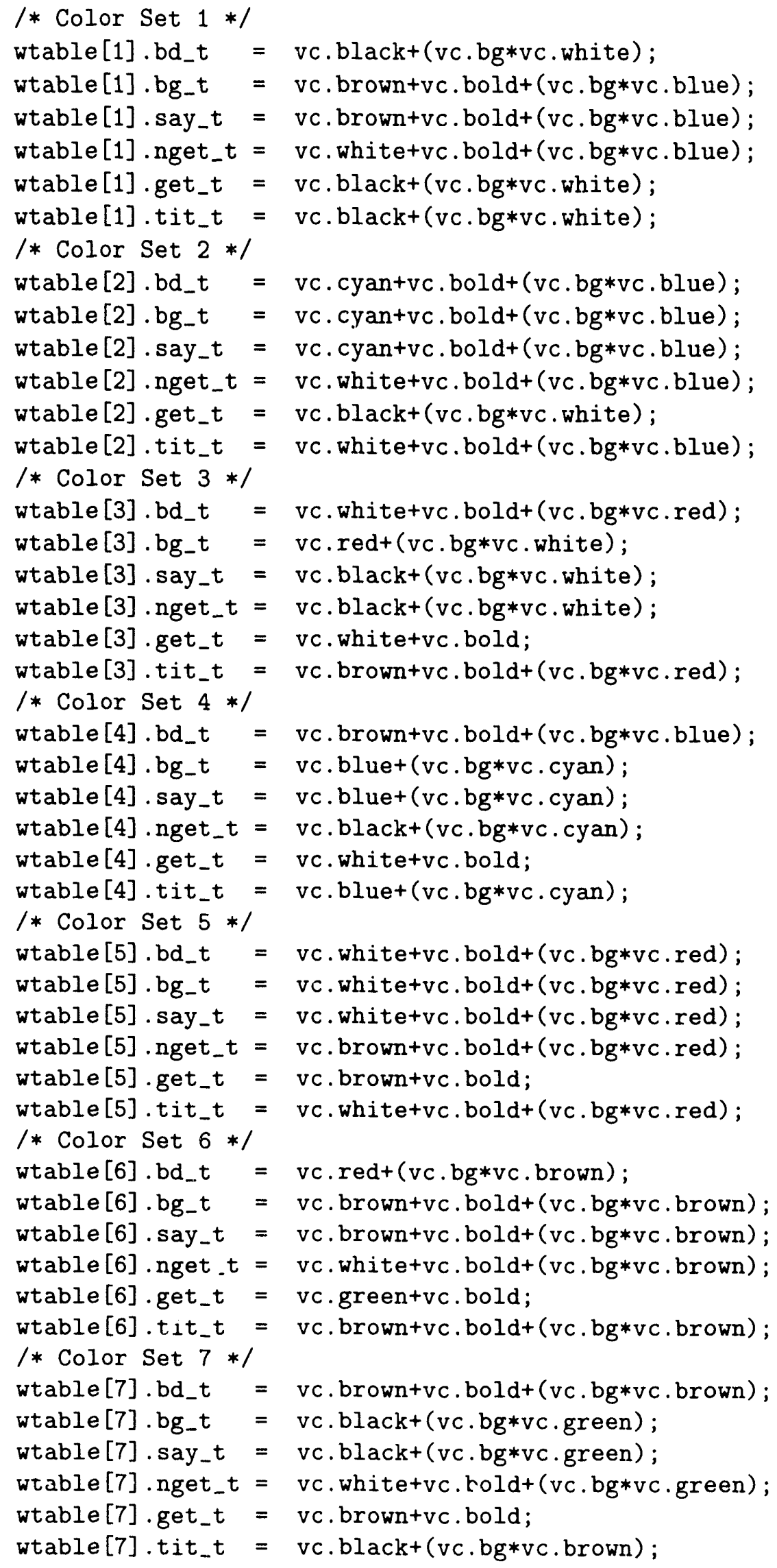




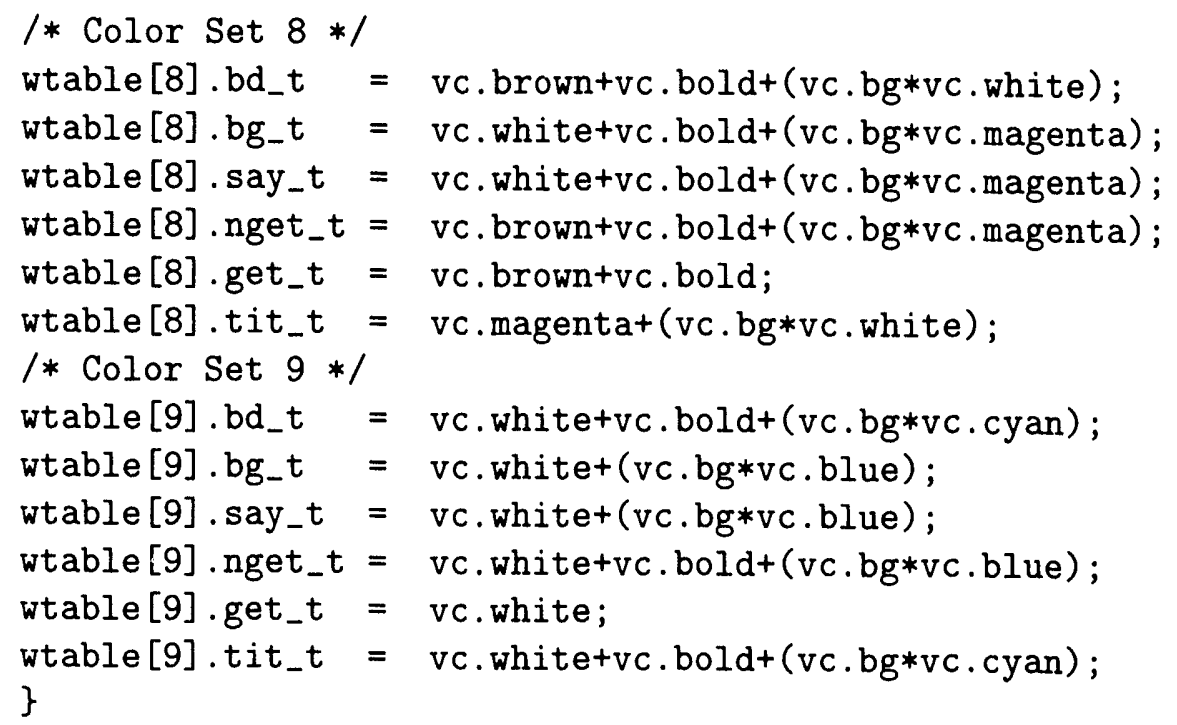




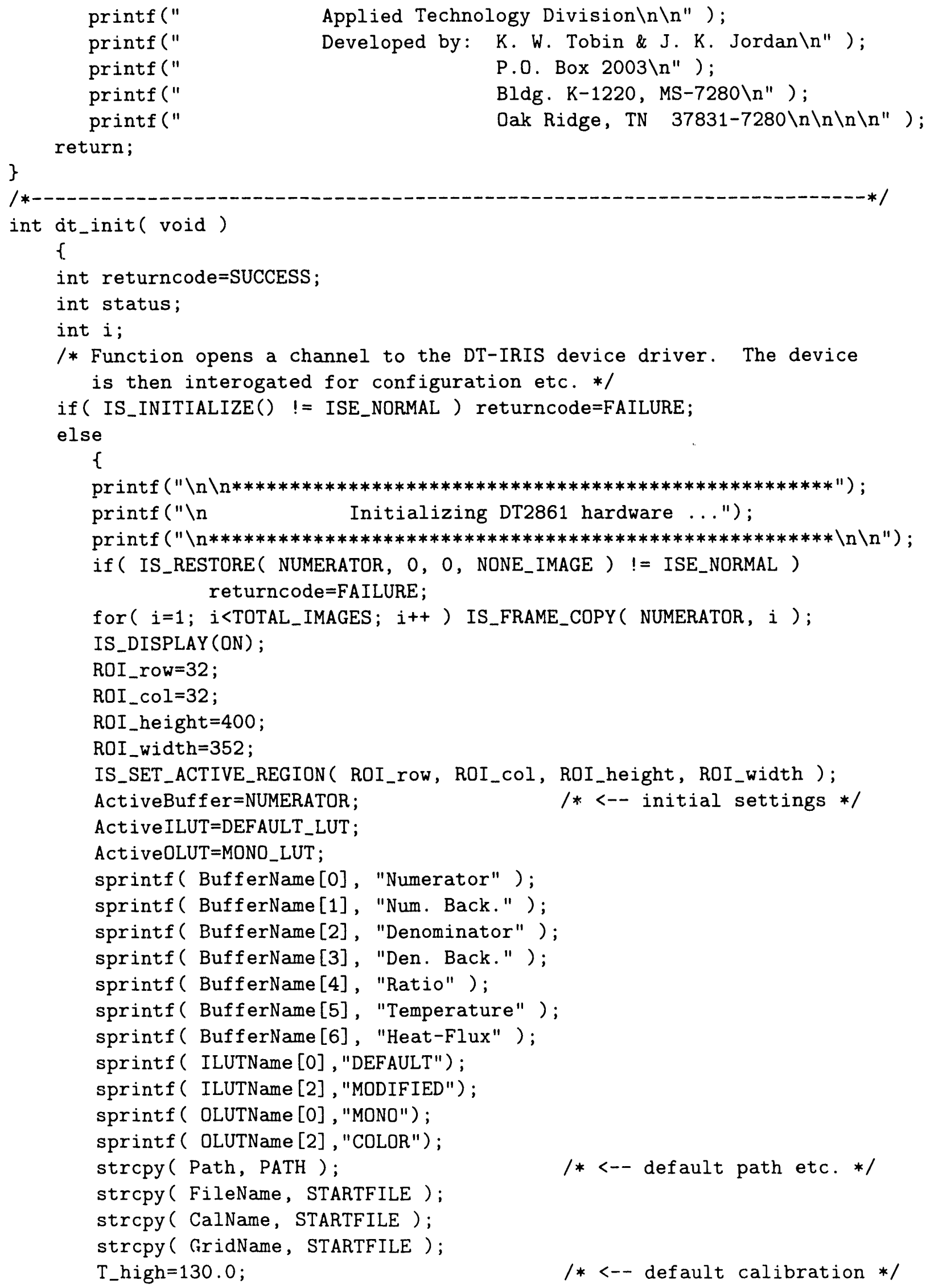




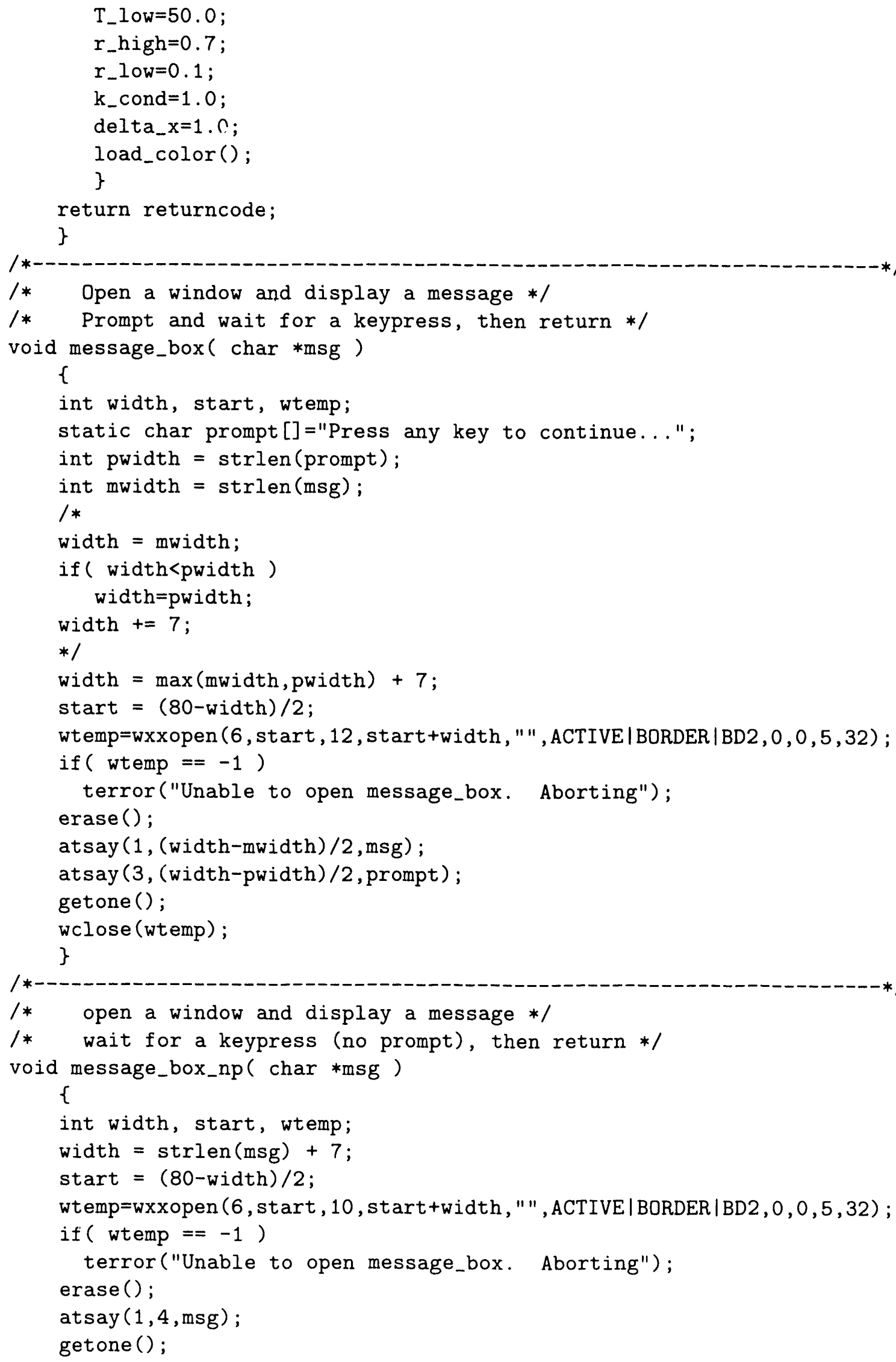




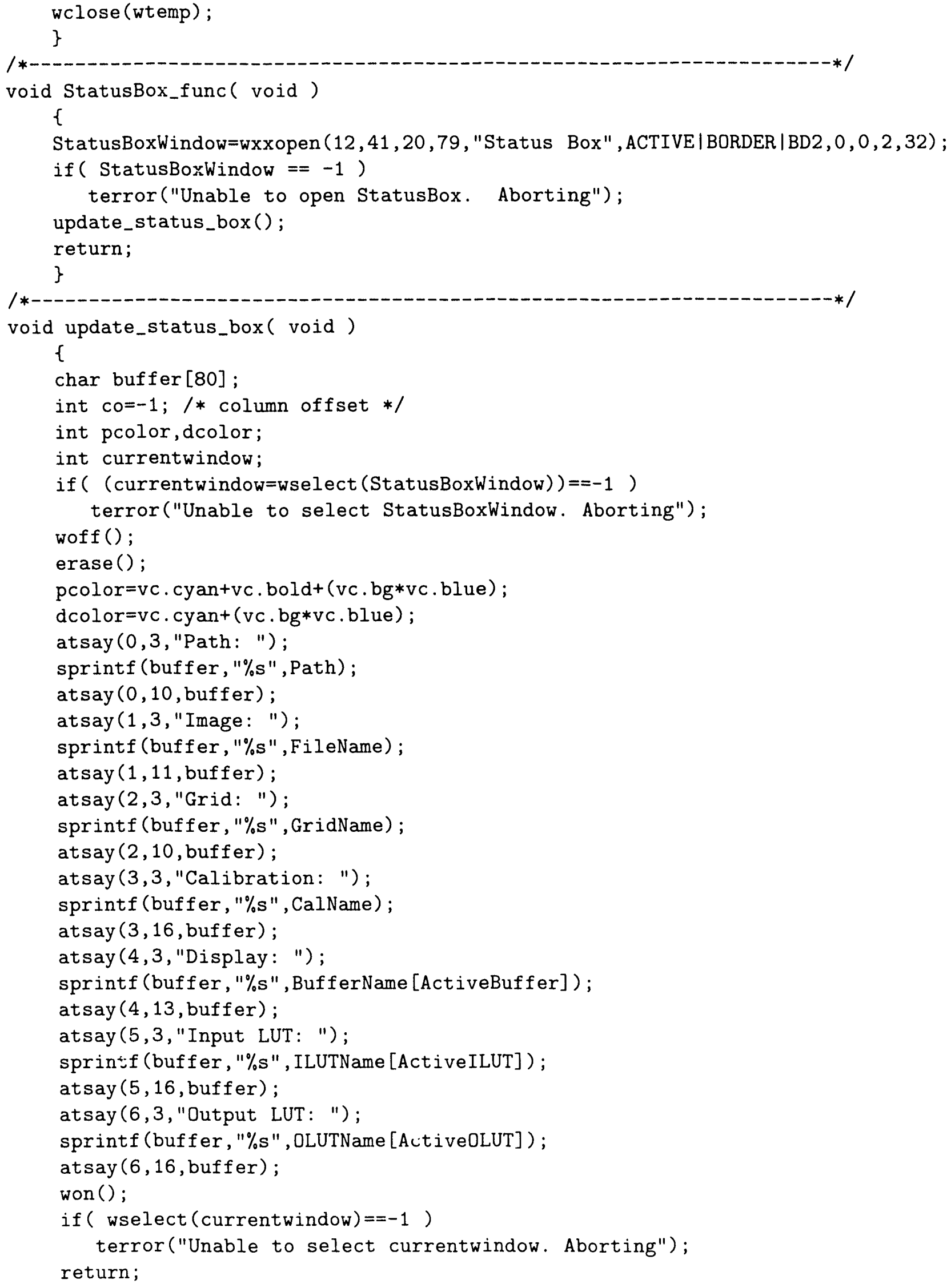




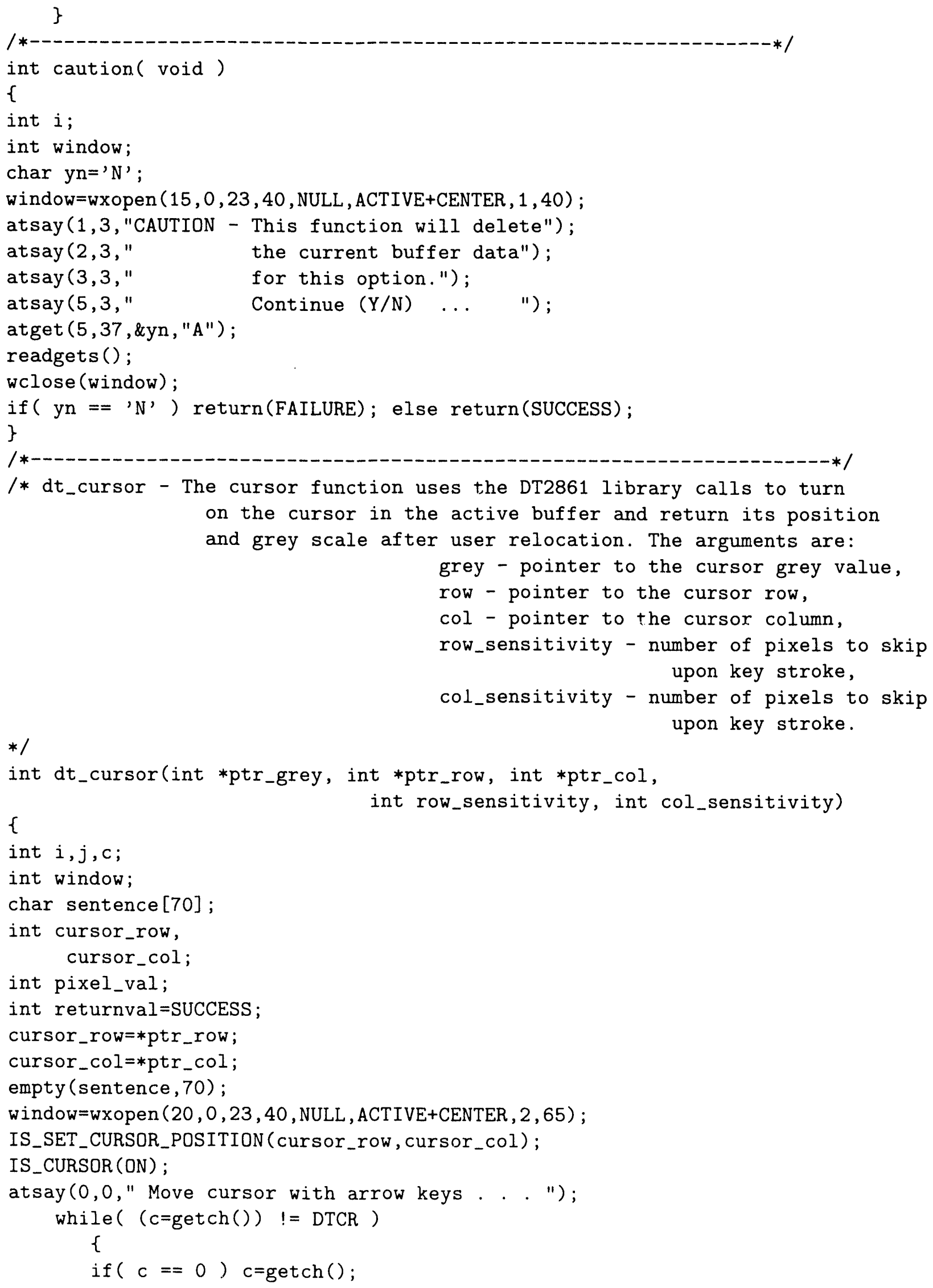




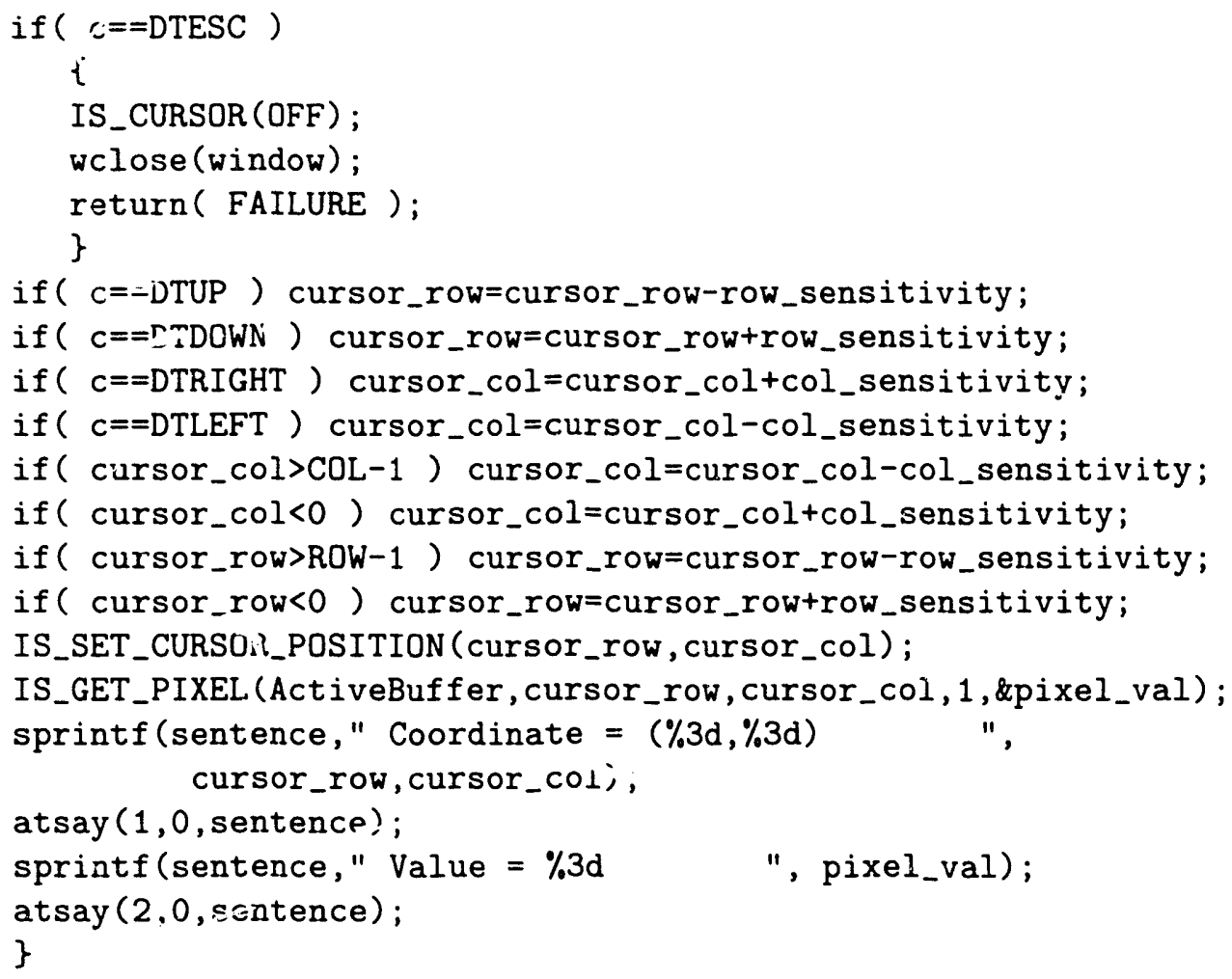




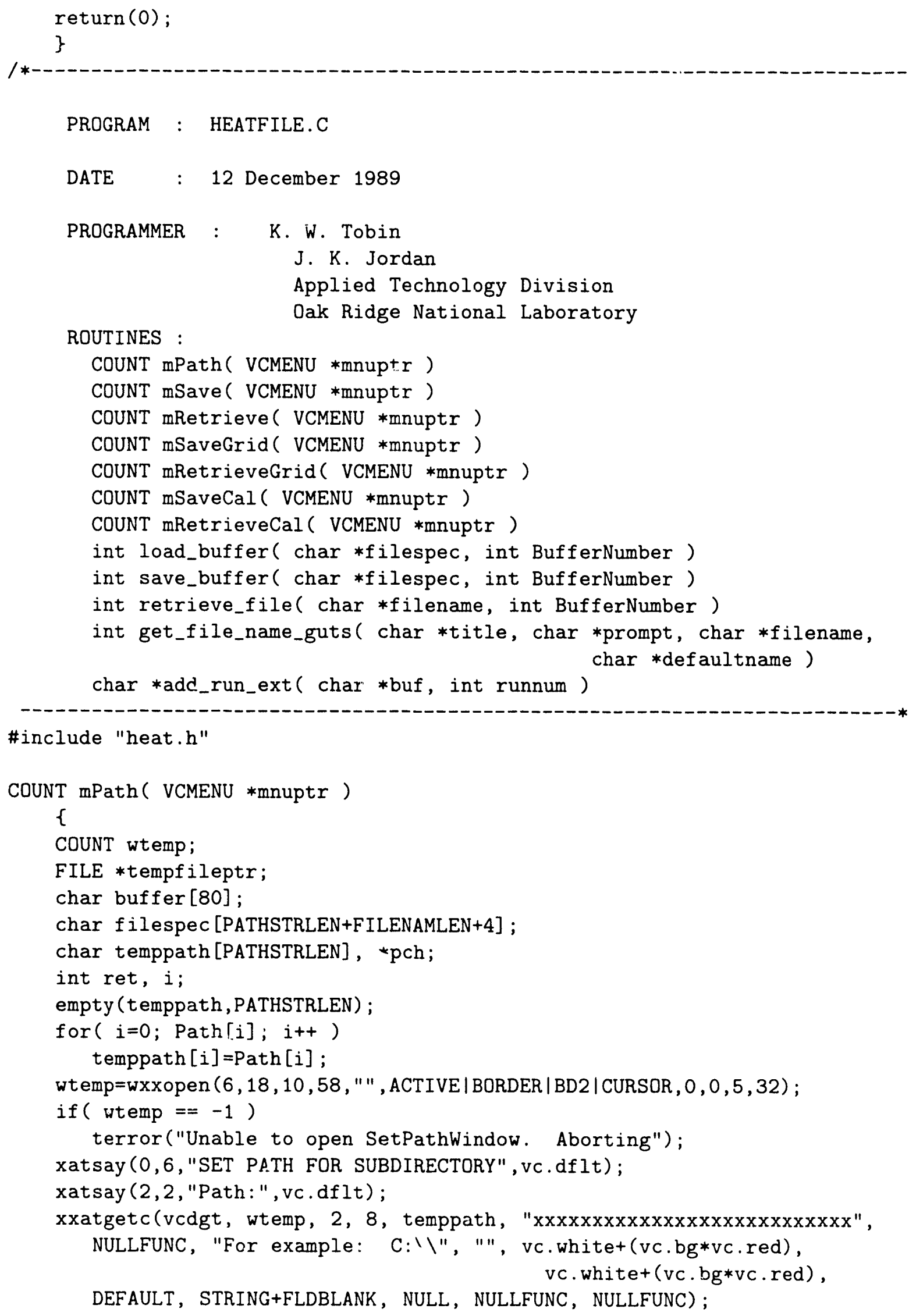




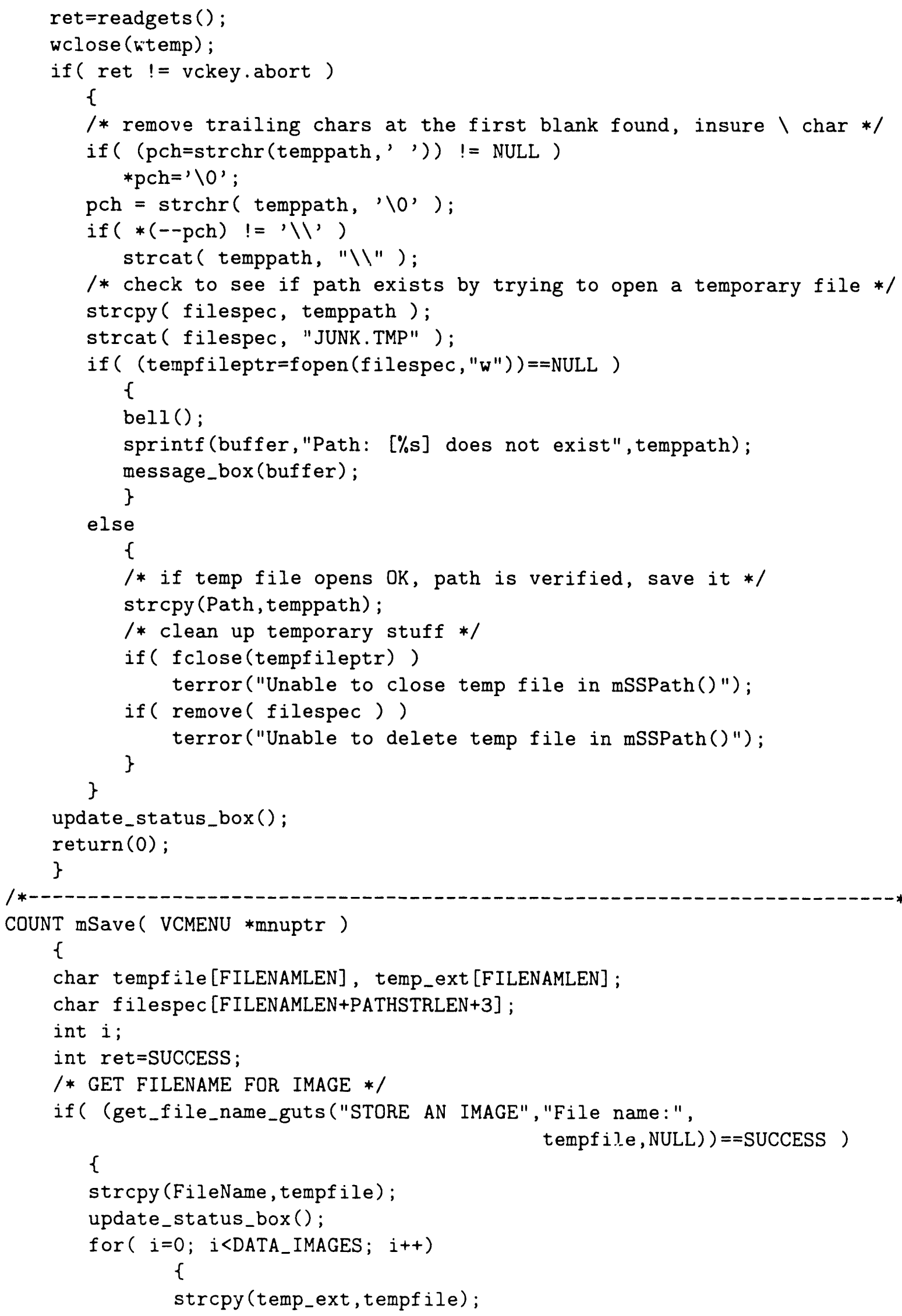




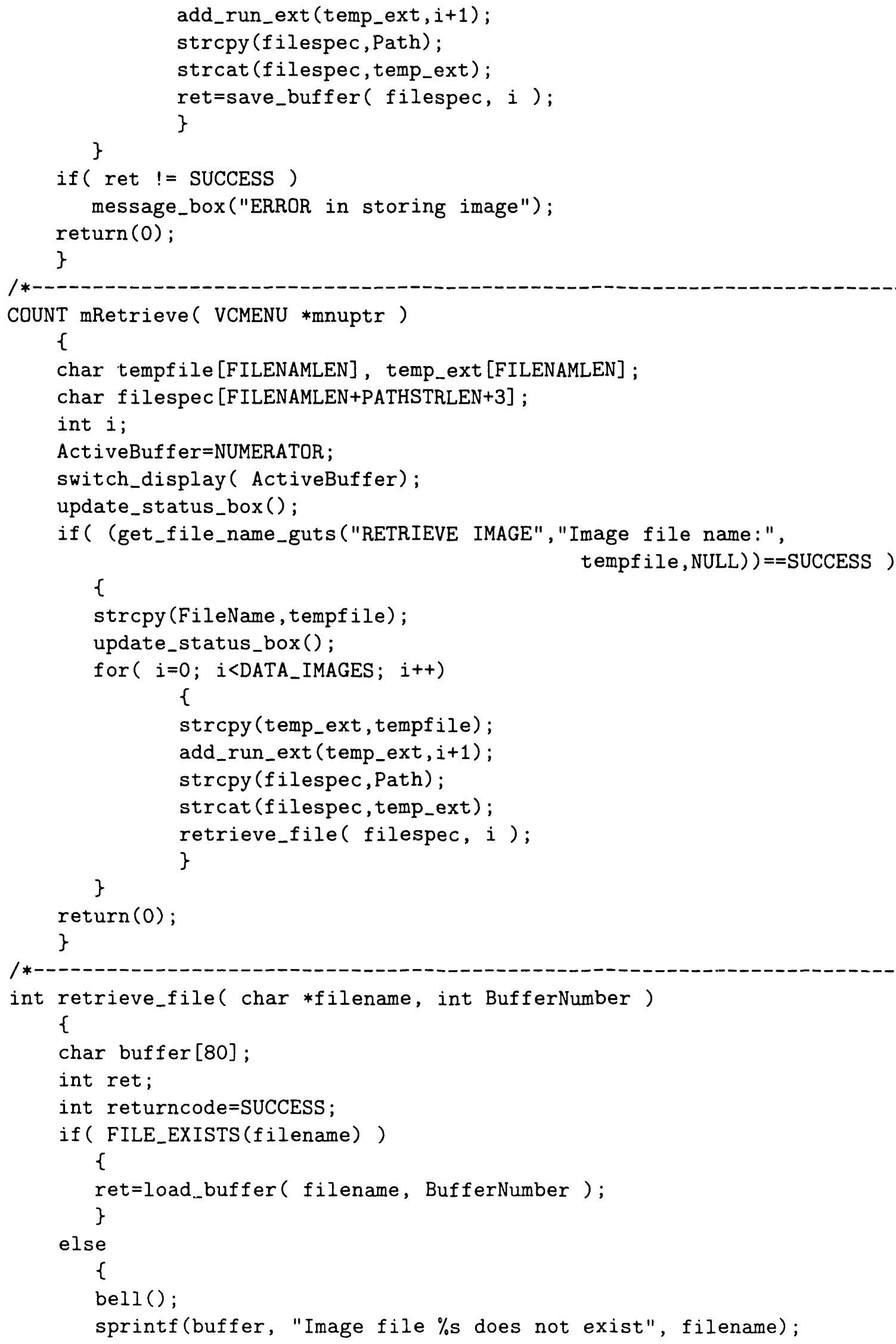




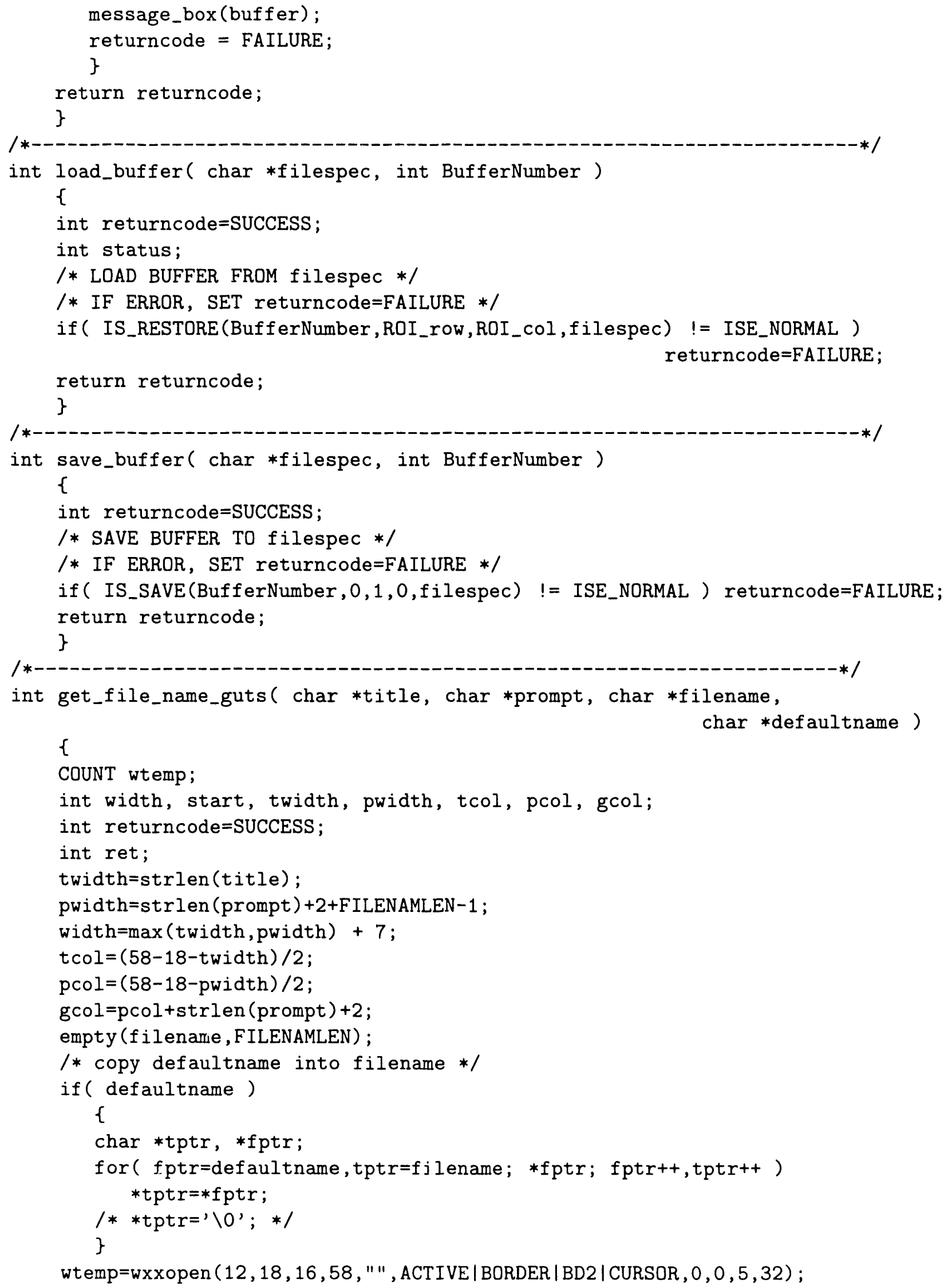




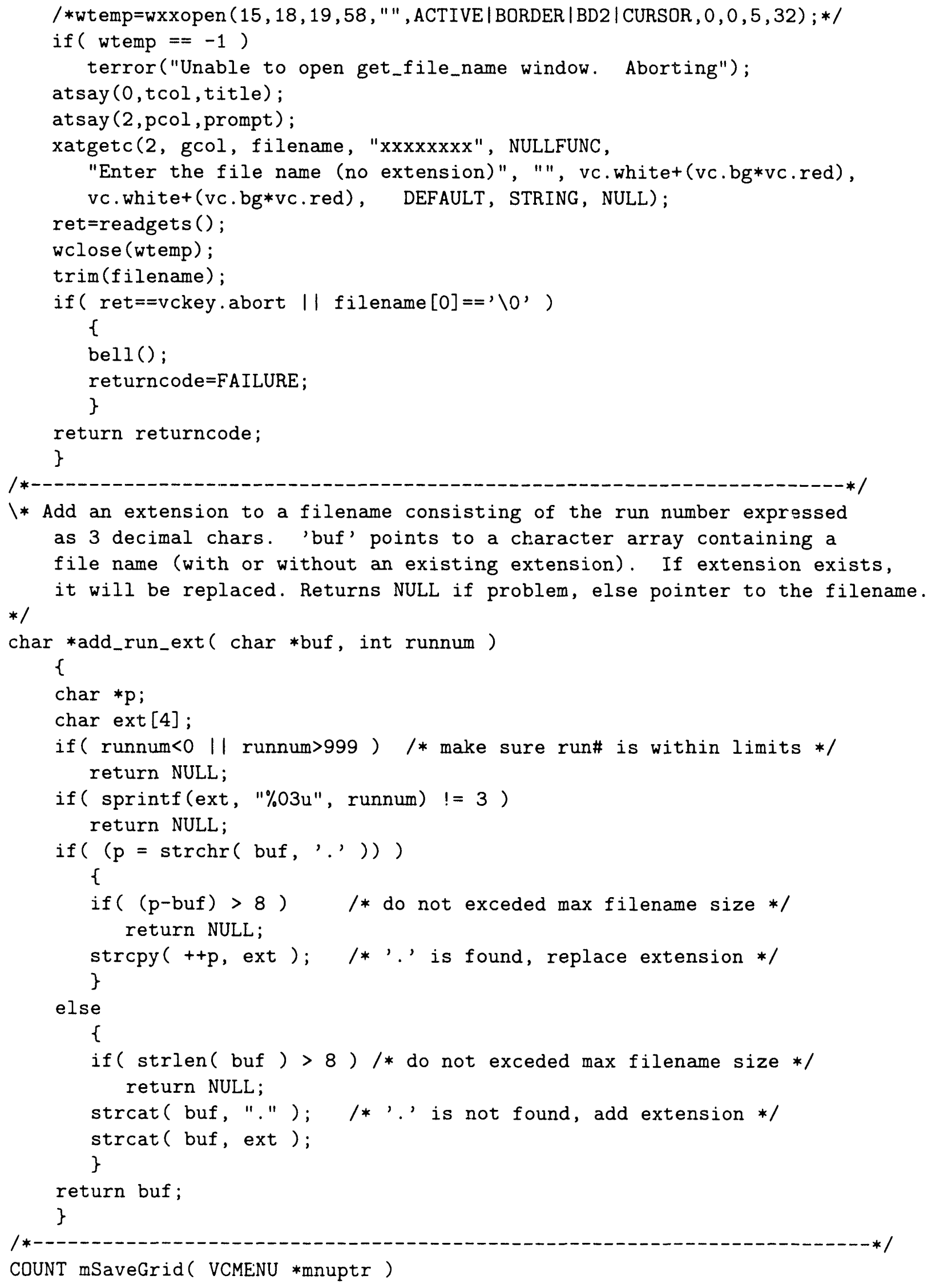




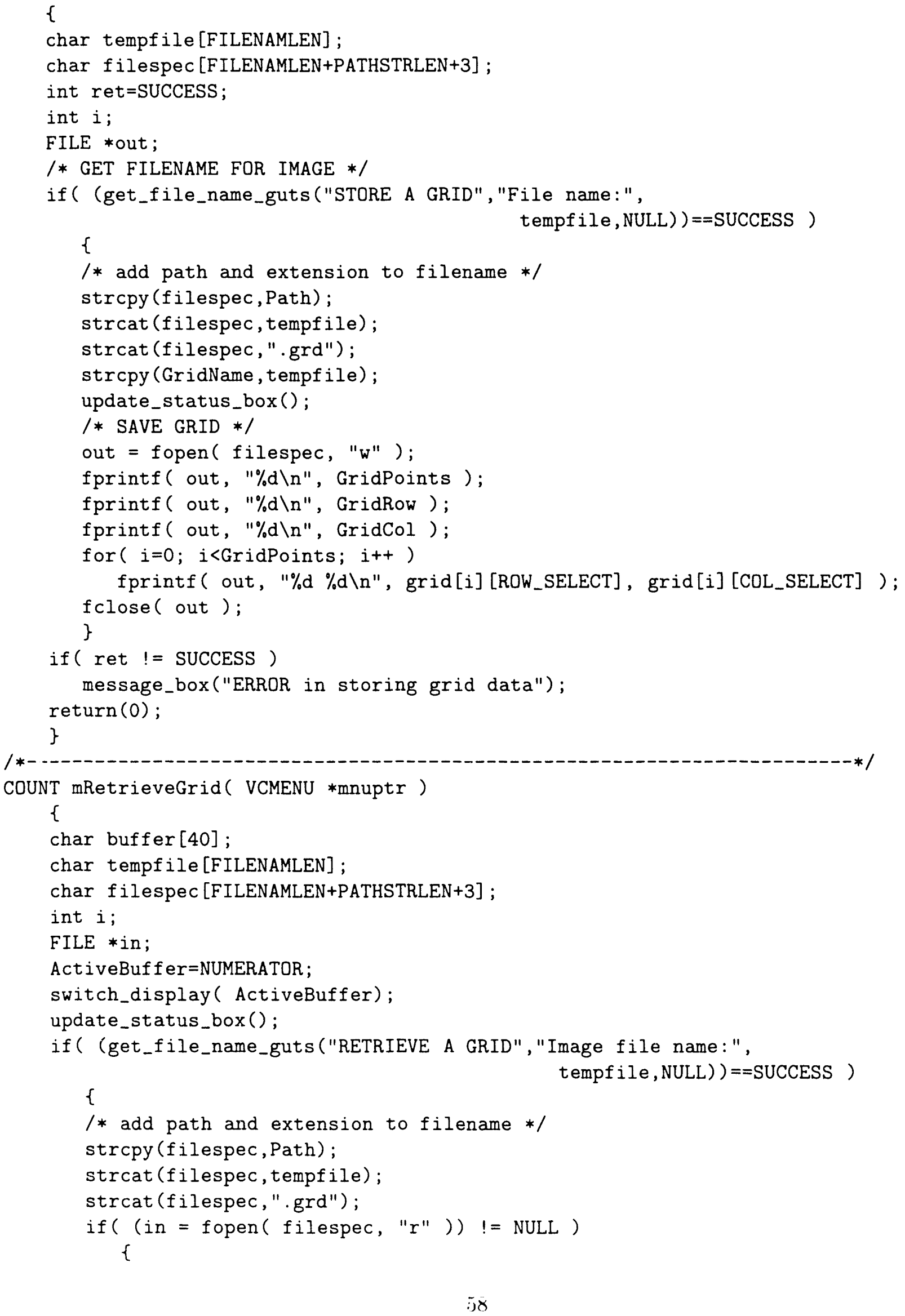




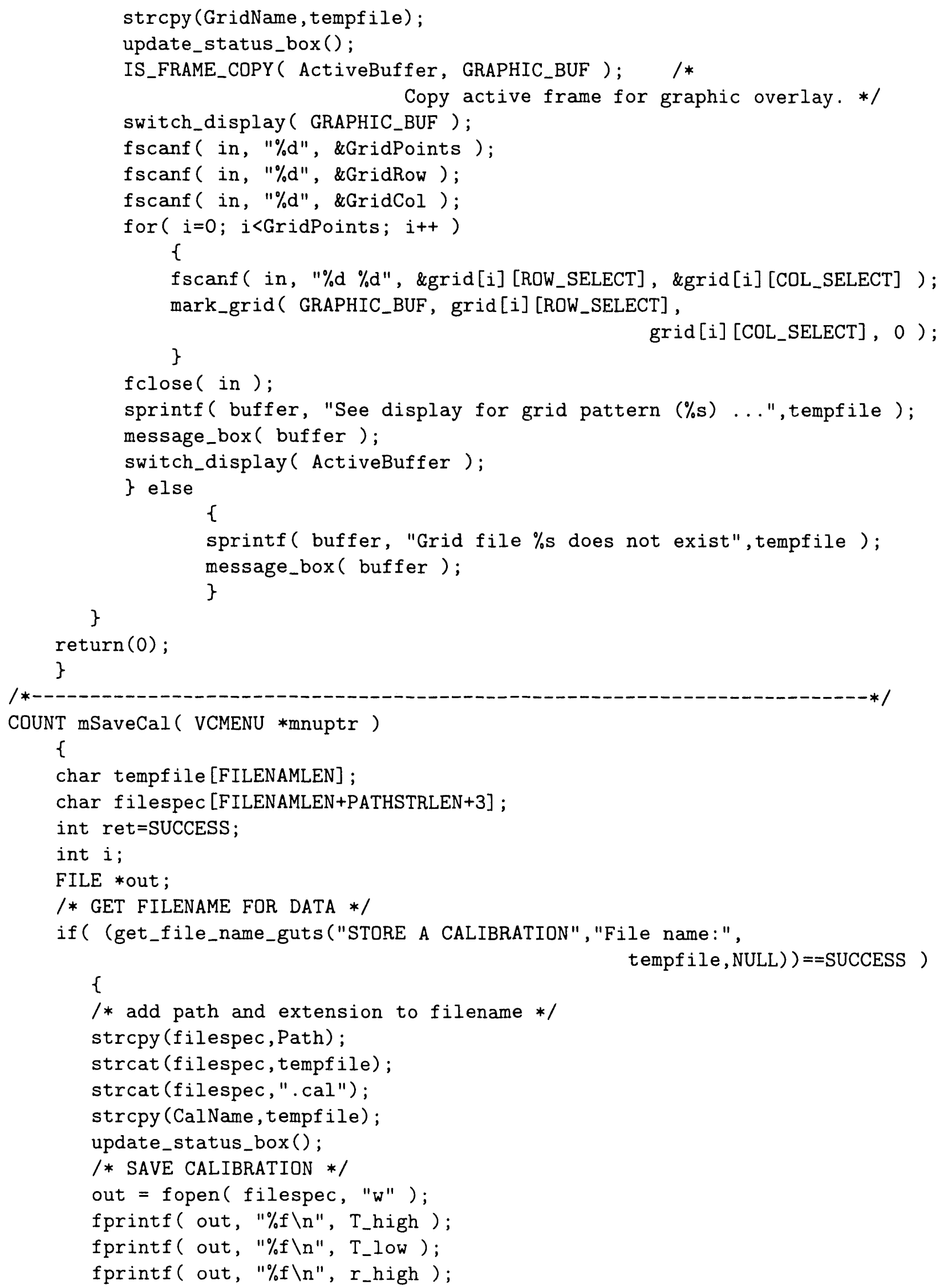




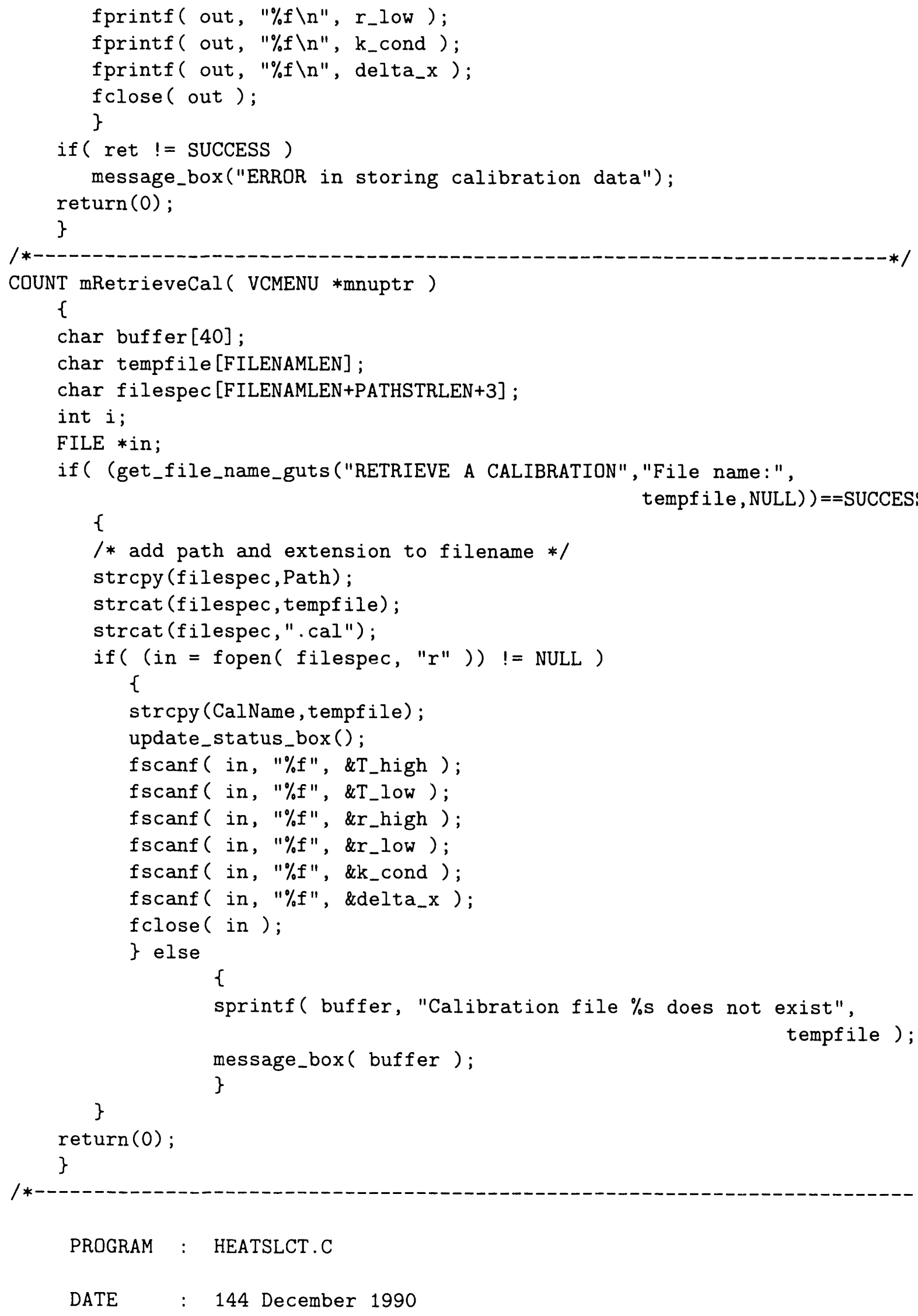




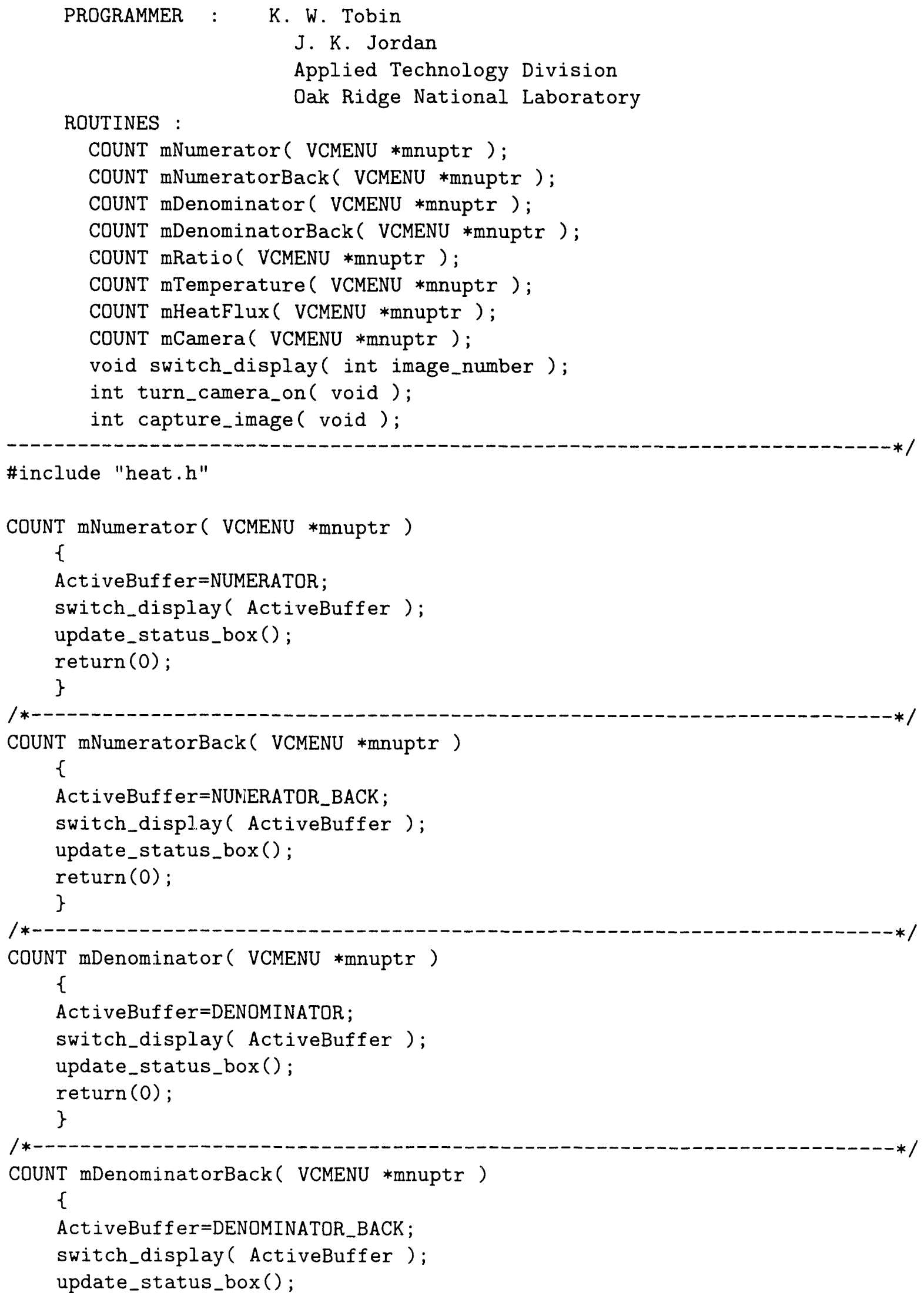




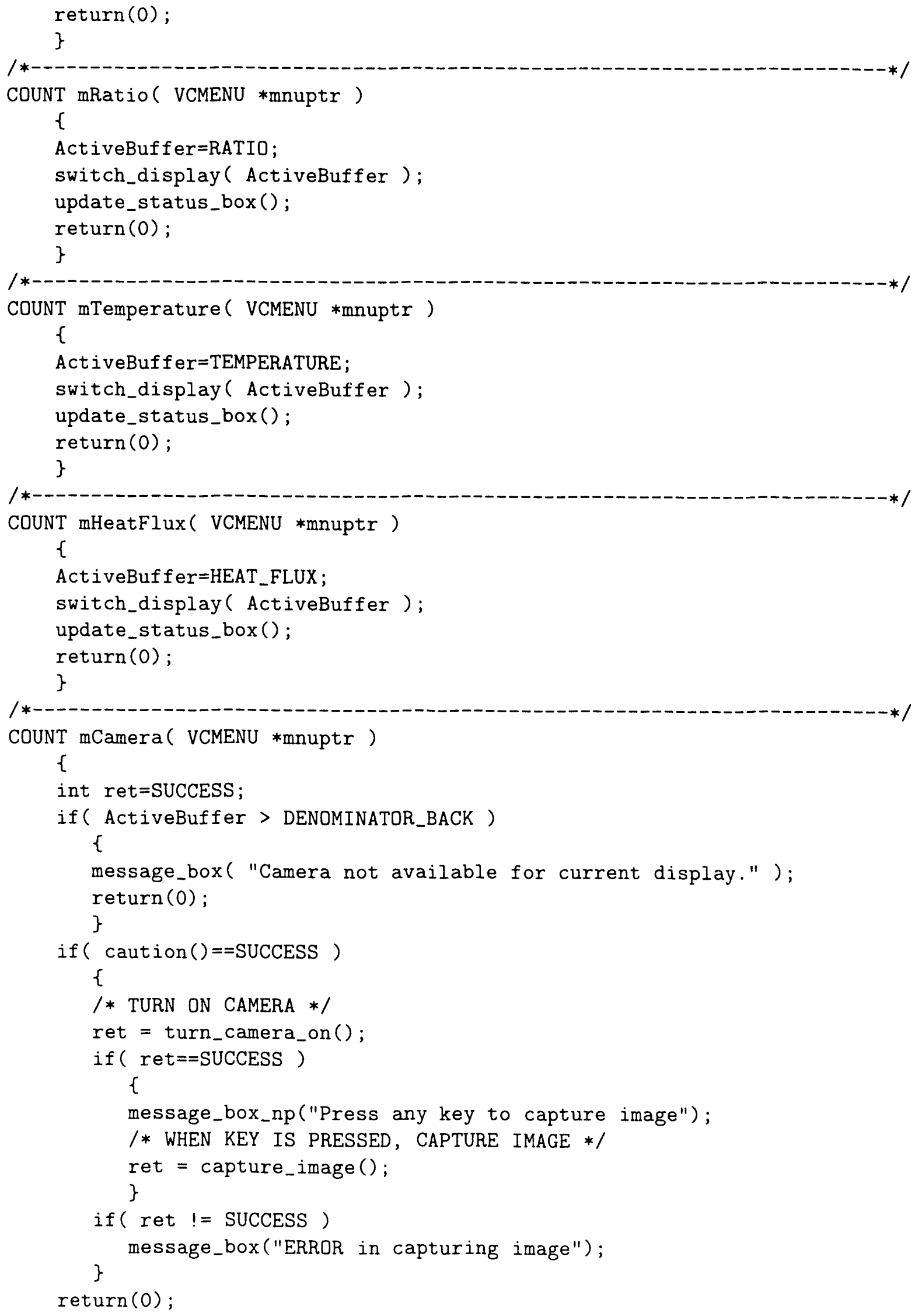




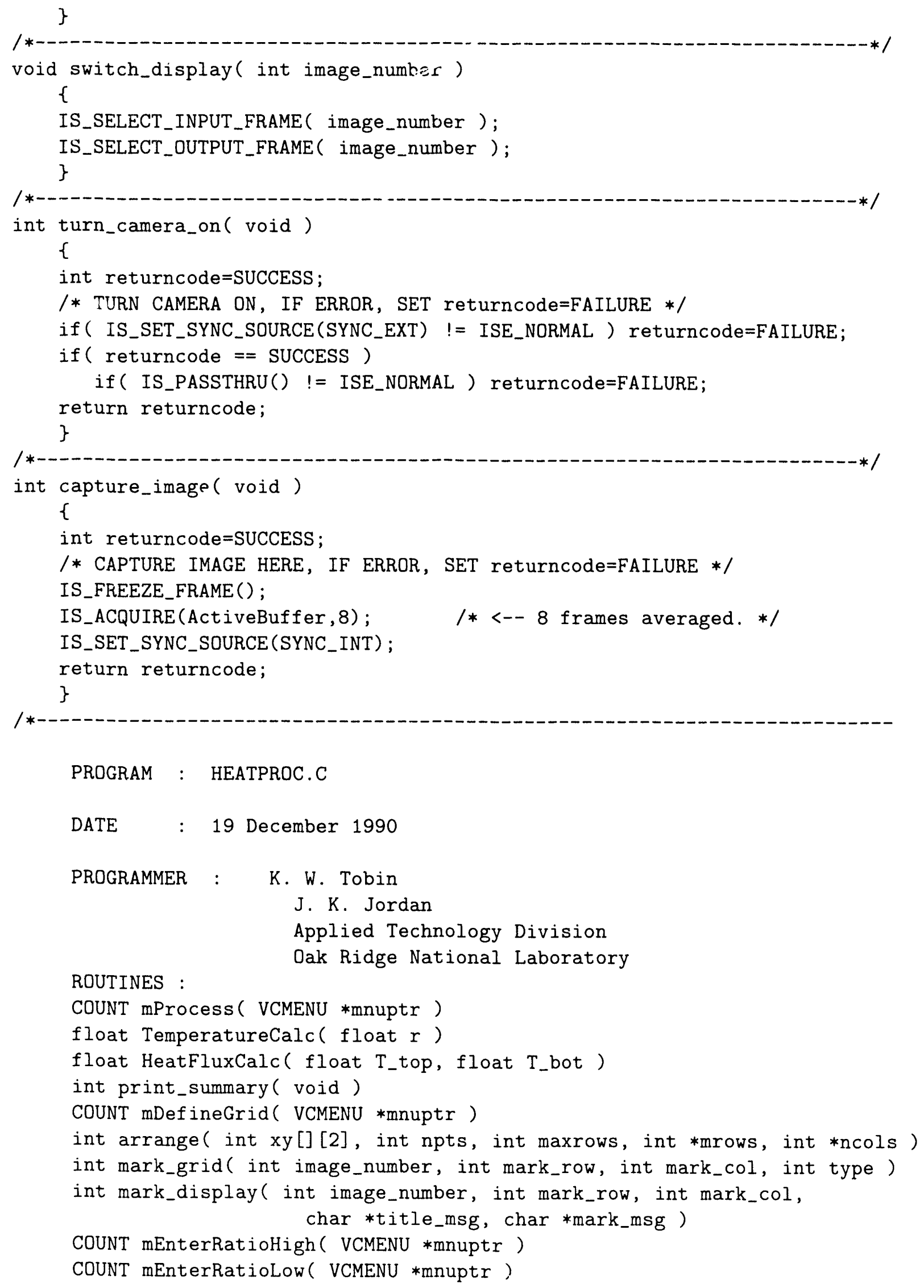


COUNT mEnterTempHigh ( VCMENU *mnuptr)

COUNT mEnterTempLow ( VCMENU *mnuptr)

COUNT mEnterk ( VCMENU *mnuptr)

COUNT mEnterx ( VCMENU *mnuptr)

void mCalibrateTemp ( void)

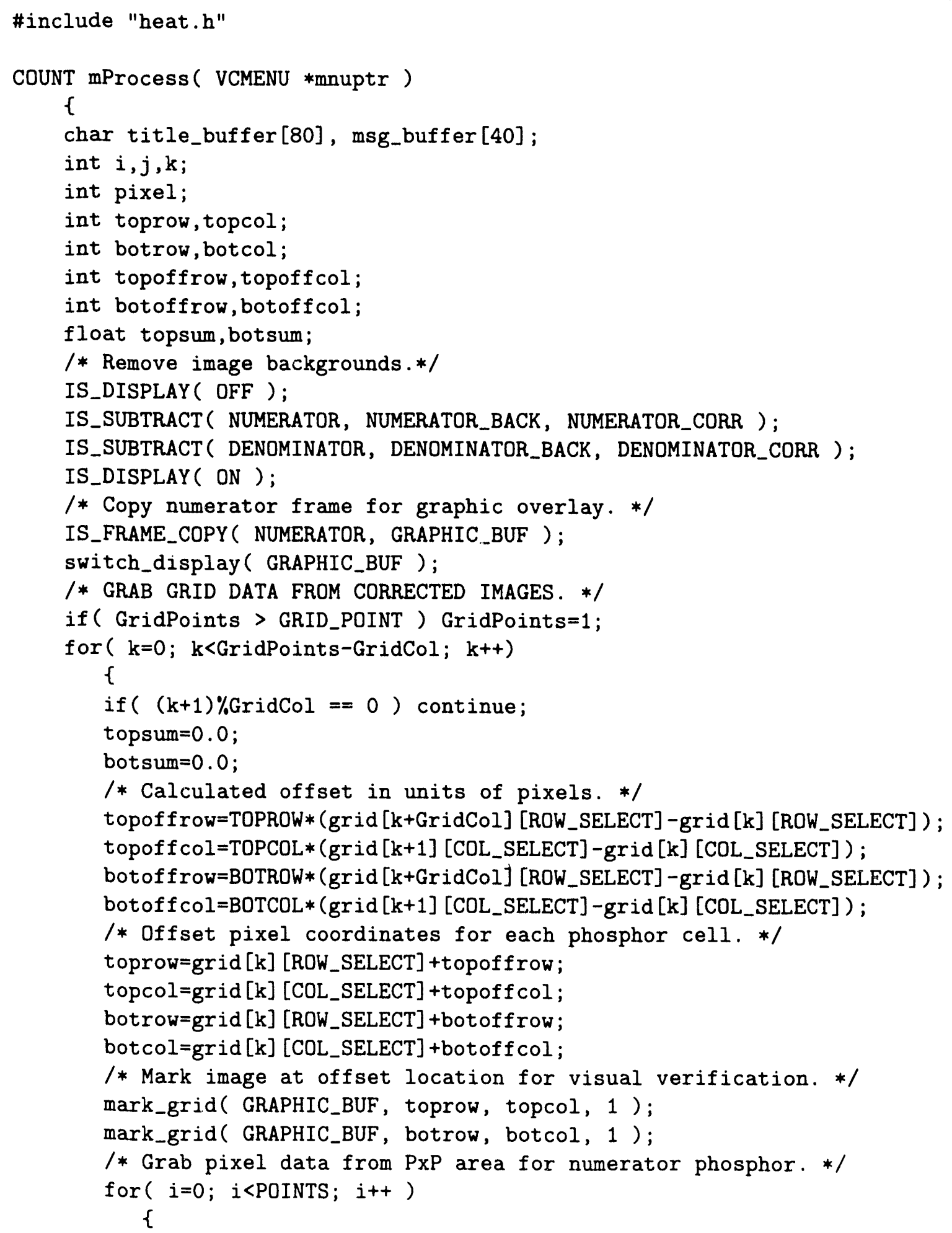




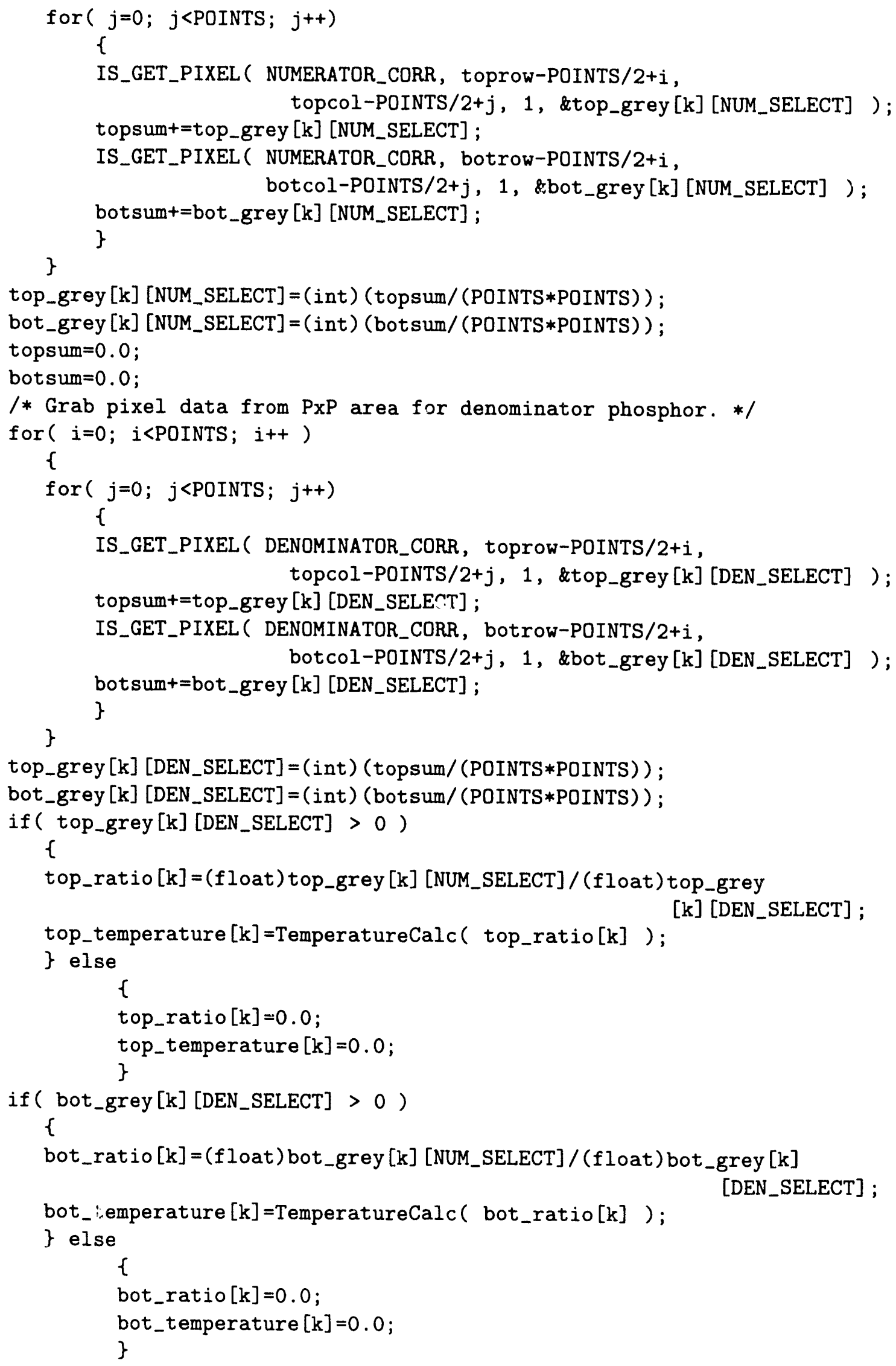


heat_flux $[\mathrm{k}]=$ HeatFluxCalc ( top_temperature [k], bot_temperature [k] ); \}

/* DISPLAY RATIO DATA ON IMAGE BUFFER */

IS_FRAME_COPY( NUMERATOR, RATIO );

ActiveBuffer=RATIO;

switch_display ( ActiveBuffer);

update_status_box();

sprintf( title_buffer, "PHOSPHOR RÁTIO DATA (front/back)" );

for ( $k=0 ; k<$ GridPoints-GridCol; $k++)$

\{

if $(k+1) \%$ GridCol $==0)$ continue;

sprintf ( msg_buffer, "\%3.2f", top_ratio[k] );

mark_display ( ActiveBuffer, grid[k] [ROW_SELECT], grid[k] [COL_SELECT], title_buffer, msg_buffer);

sprintf ( msg_buffer, "\%3.2f", bot_ratio[k]) ;

mark_display ( ActiveBuffer, grid[k] [ROW_SELECT]+17, grid [k] [COL_SELECT] +5, title_buffer, msg_buffer );

\}

/* DISPLAY TEMPERATURE DATA ON IMAGE BUFFER */

IS_FRAME_COPY ( NUMERATOR, TEMPERATURE );

ActiveBuffer=TEMPERATURE;

switch_display ( ActiveBuffer);

update_status_box();

sprintf ( title_buffer, "PHOSPHOR TEMPERATURE DATA (\%s)", DTTUNIT);

for $(k=0 ; k<G r i d P o i n t s-G r i d C o l ; k++)$

\{

if $(\mathrm{k}+1) \%$ GridCol $==0)$ continue;

sprintf (msg_buffer, "\%4.1f", top_temperature[k]) ;

mark_display (ActiveBuffer, grid[k] [ROW_SELECT], grid[k] [COL_SELECT], title_buffer, msg_buffer);

sprintf (msg_buffer, "\%4.1f", bot_temperature[k]);

mark_display ( ActiveBuffer, grid[k] [ROW_SELECT]+17, grid[k] [COL_SELECT] +5, title_buffer, msg_buffer );

\}

/* DISPLAY HEAT FLUX DATA ON IMAGE BUFFER */

IS_FRAME_COPY ( NUMERATOR, HEAT_FLUX );

ActiveBuffer=HEAT_FLUX;

swi.tch_display (ActiveBuffer);

urdate_status_box ();

sprintf( title_'Juffer, "PHOSPHOR HEAT FLUX DATA (\%s)", DTQUNIT);

for $(k=1) ; k r G$ idPcints-GridCol; $k++)$

\{

if $(\mathrm{k}+1) \%$ GridCol $==0)$ continue;

sprintf ( msg_buffer, "\%4.1f", heat_flux[k] );

mark_display ( ActiveBuffer, grid[k] [ROW_SELECT], grid[k] [COL_SELECT], title_buffer, msg_buffer);

\}

print_summary ();

\} 


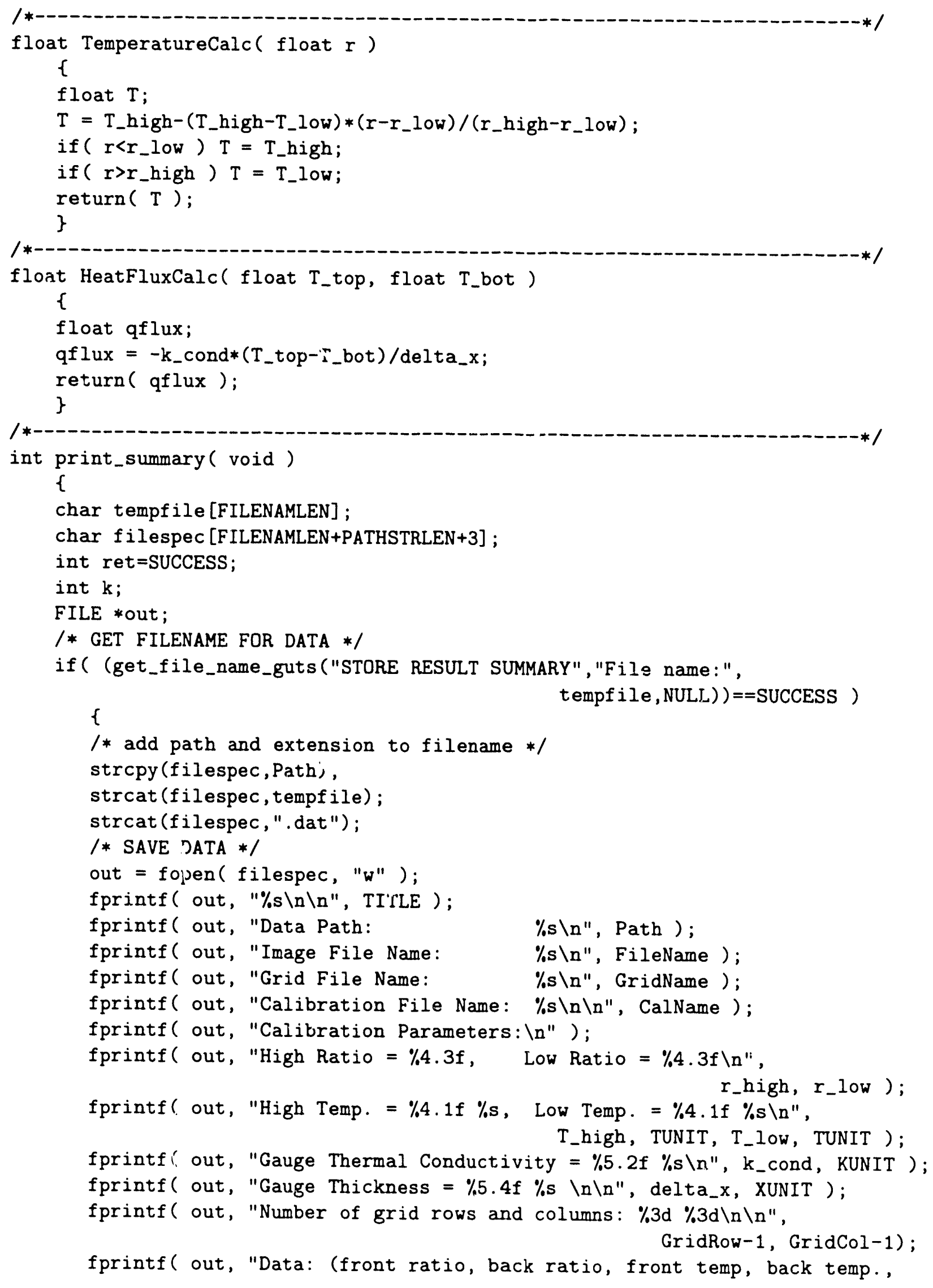


for $(k=0 ; k<$ GridPoints-GridCol; $k++)$

$$
\{
$$




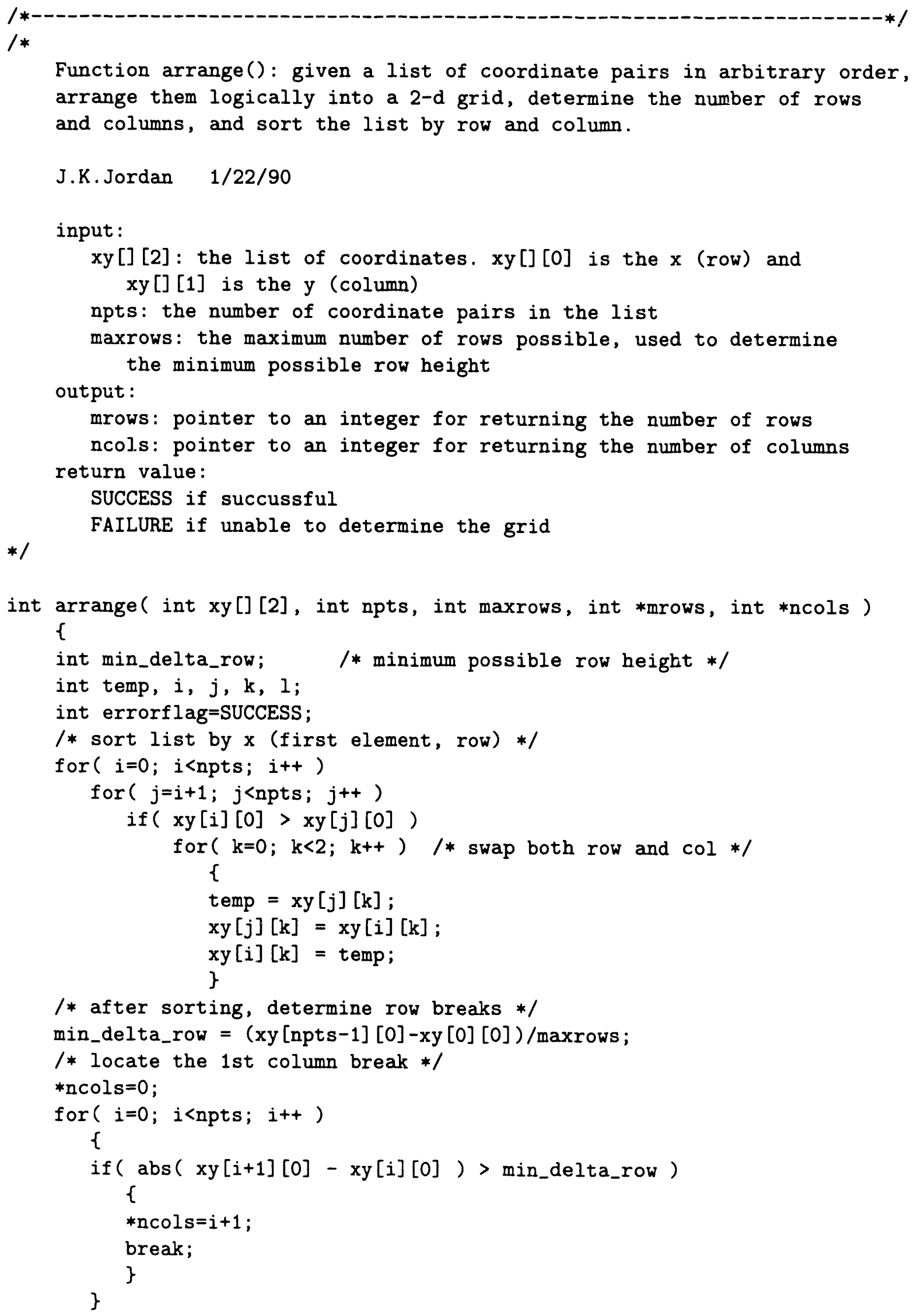
arrange them logically into a 2-d grid, determine the number of rows and columns, and sort the list by row and column.

\section{J.K.Jordan $1 / 22 / 90$}

input :

$x y[][2]$ : the list of coordinates. $x y[][0]$ is the $x$ (row) and xy [] [1] is the $y$ (column)

npts: the number of coordinate pairs in the list

maxrows: the maximum number of rows possible, used to determine the minimum possible row height output :

mrows: pointer to an integer for returning the number of rows ncols: pointer to an integer for returning the number of columns return value:

SUCCESS if succussful

*/

FAILURE if unable to determine the grid 


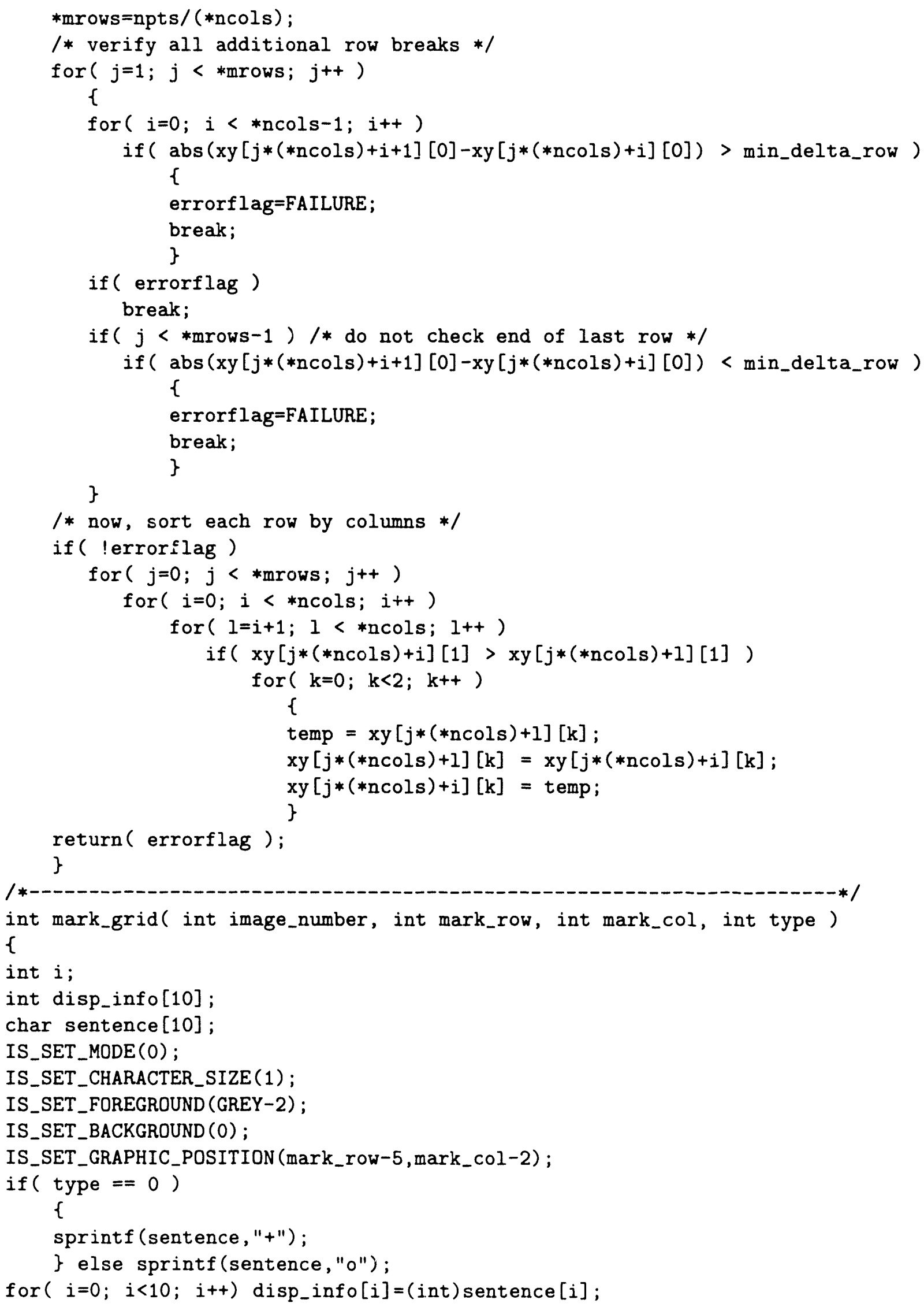




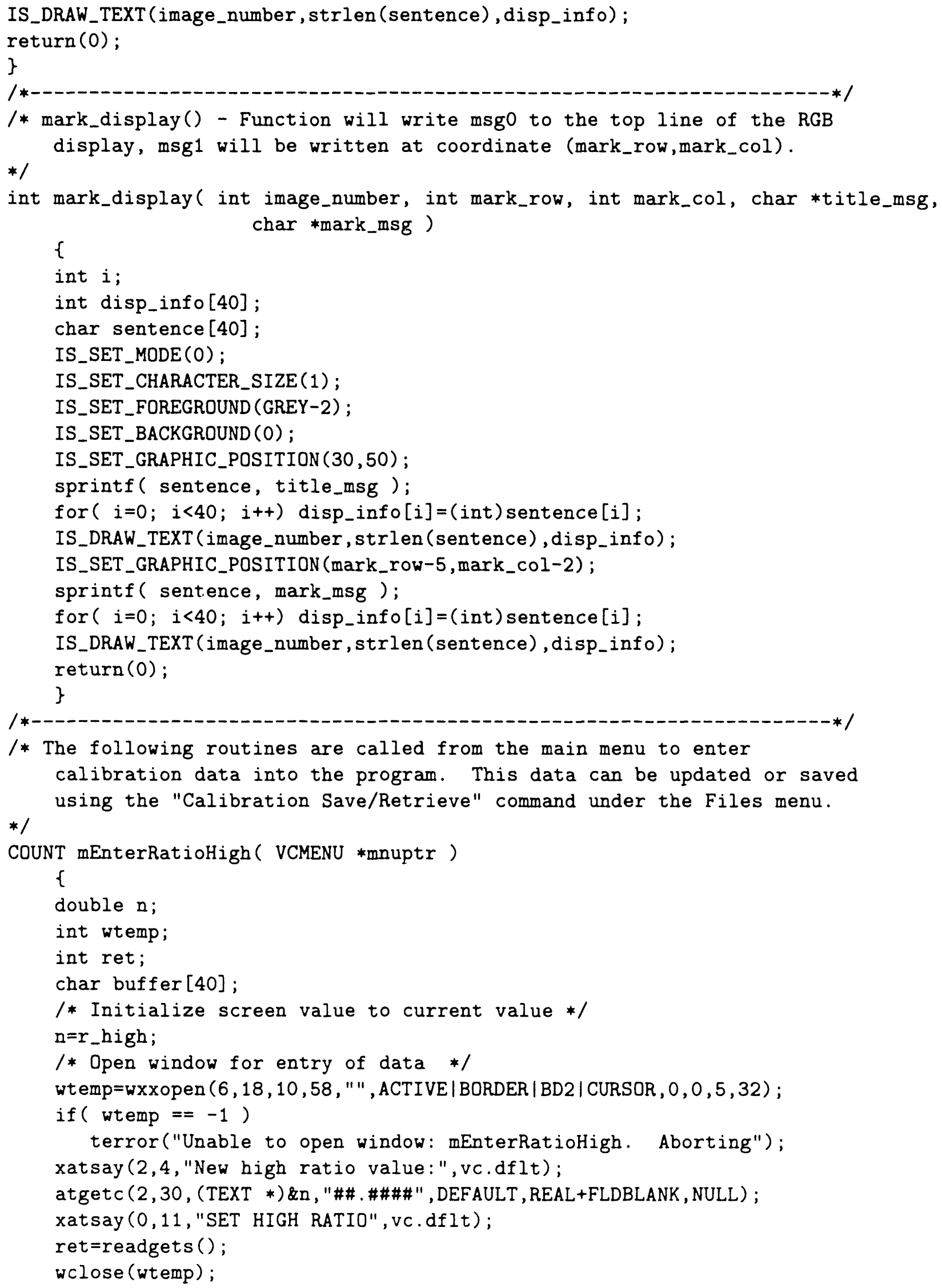

/* The following routines are called from the main menu to enter calibration data into the program. This data can be updated or saved using the "Calibration Save/Retrieve" command under the Files menu. 


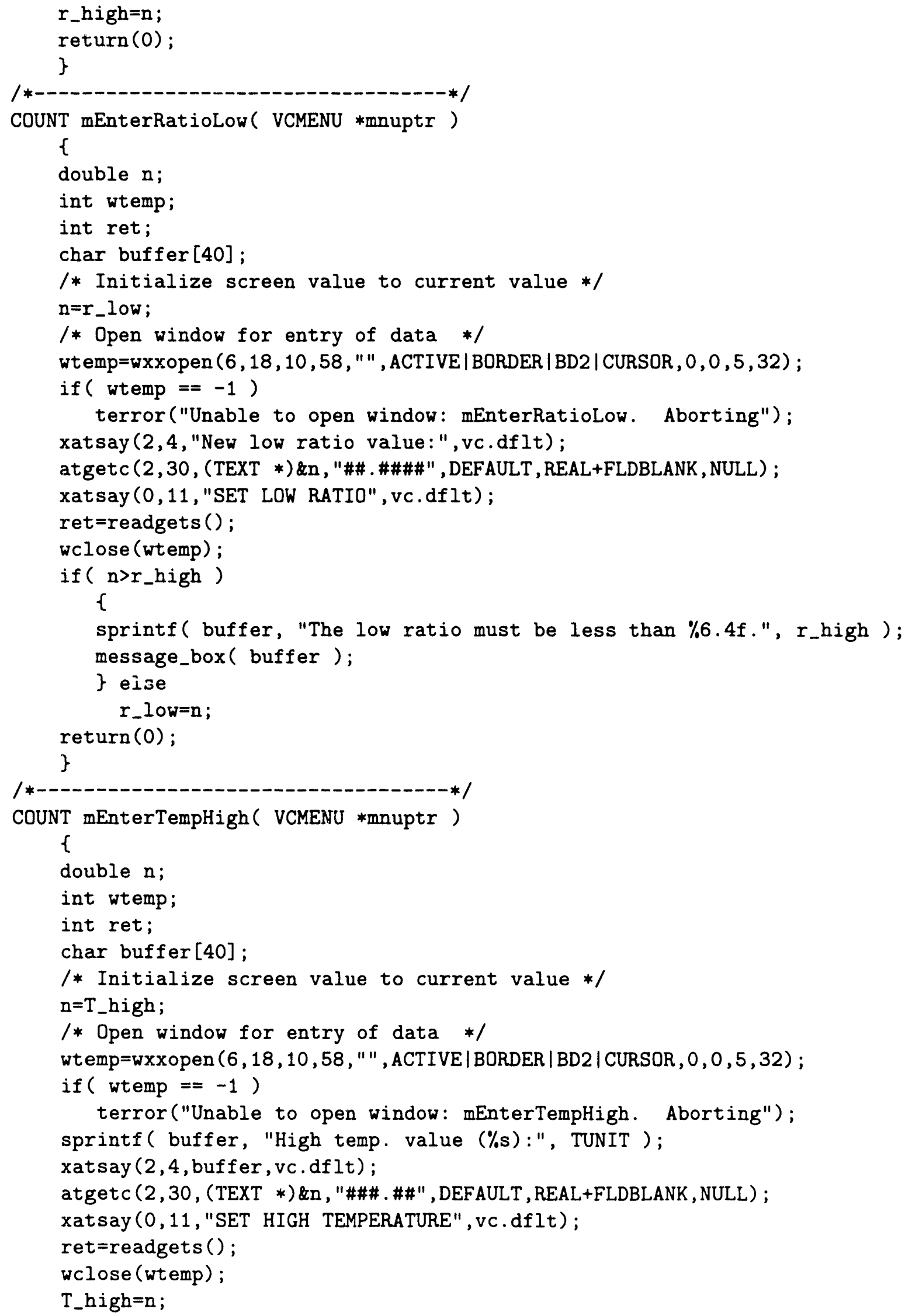




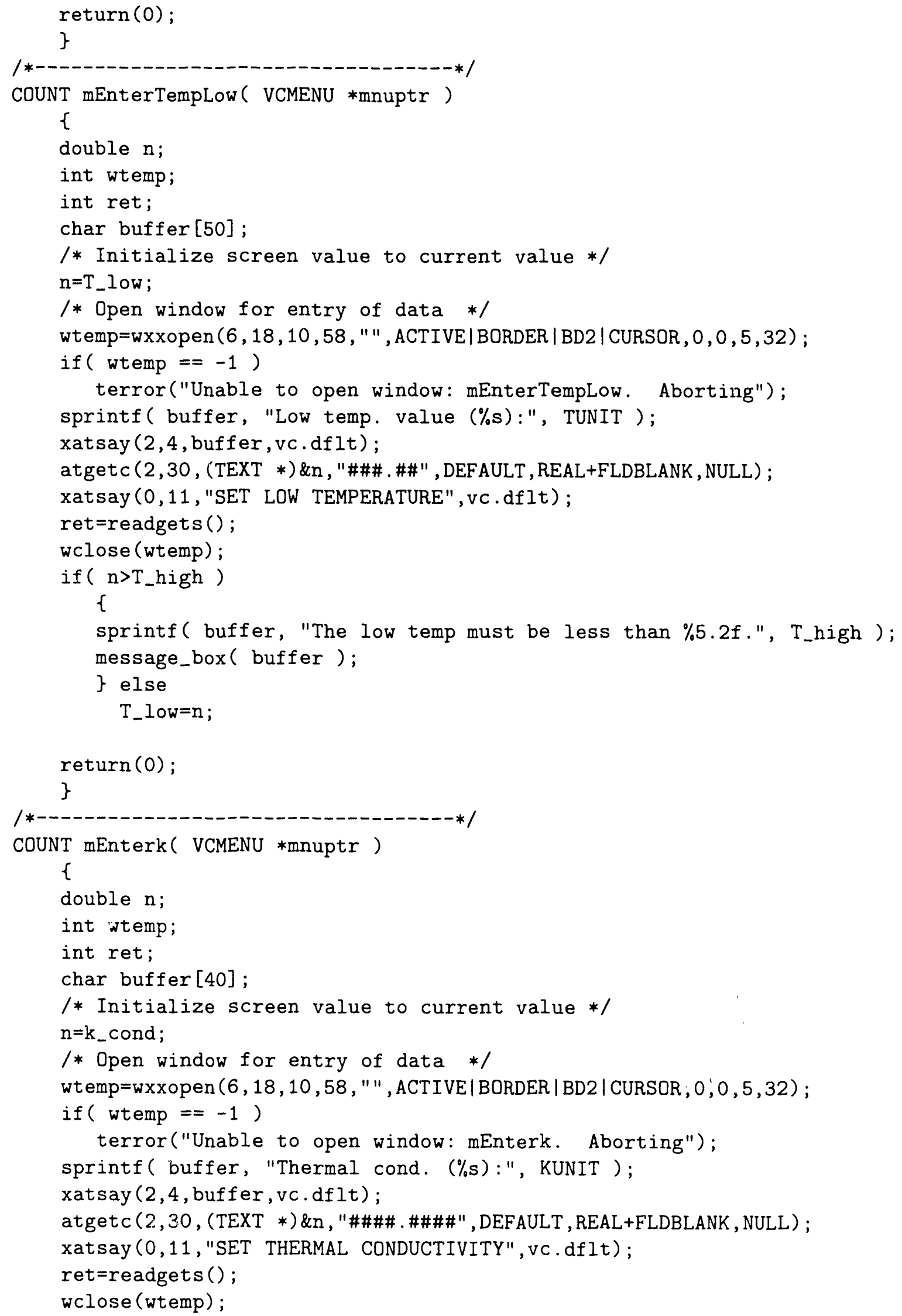




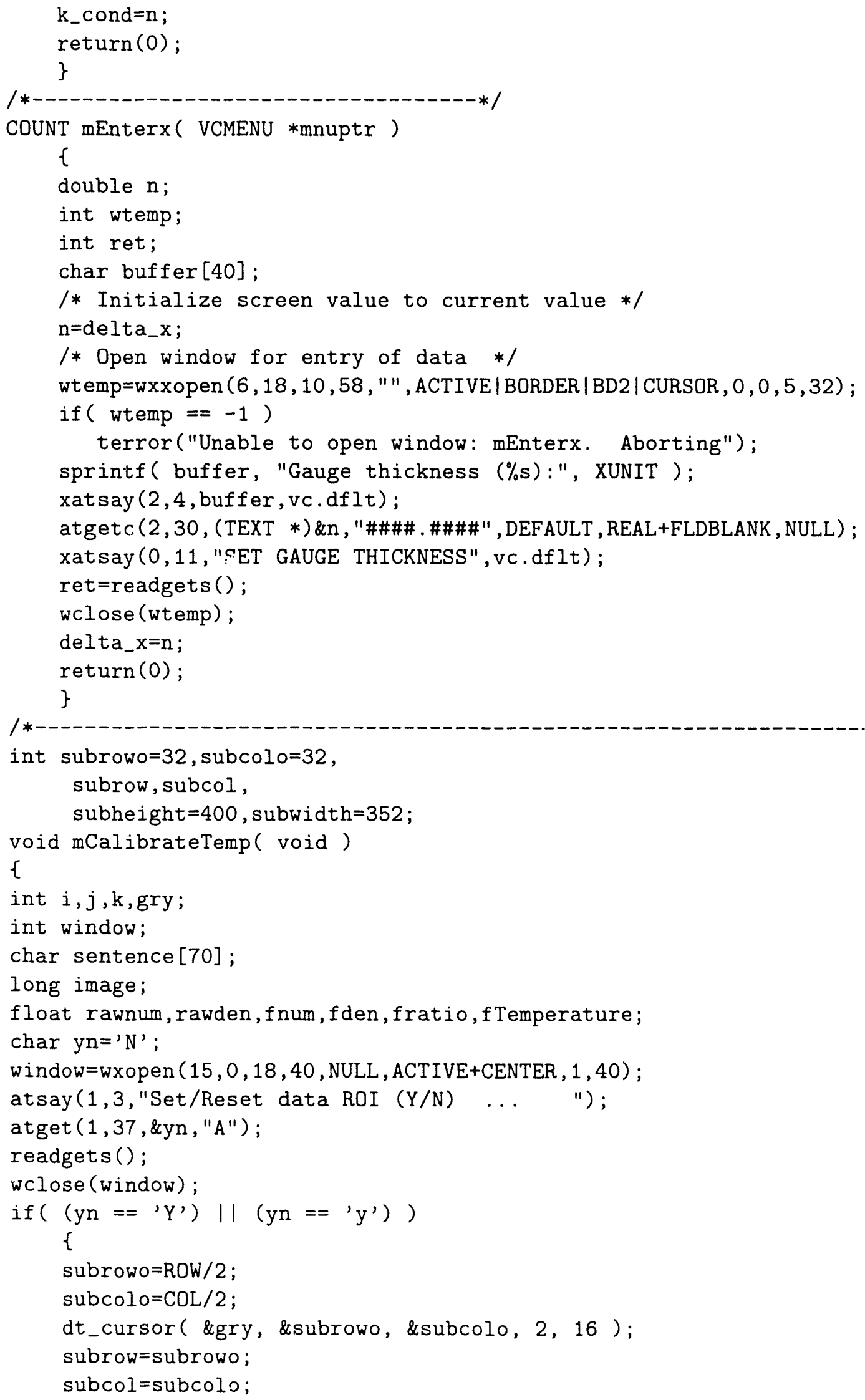


dt_cursor ( \&gry, \&subrow, \&subcol, 2, 16 );

subheight=subrow-subrowo;

subwidth=subcol-subcolo;

\}

if $($ (subheight $==0)||($ subwidth $==0))$

\{

message_box ( "Invalid range on data ROI");

return;

\} else

IS_SET_ACTIVE_REGION (subrowo, subcolo, subheight, subwidth);

/* Remove image backgrounds. */

IS_DISPLAY ( OFF );

IS_SUBTRACT( NUMERATOR, NUMERATOR_BACK, NUMERATOR_CORR );

IS_SUBTRACT( DENOMINATOR, DENOMINATOR_BACK, DENOMINATOR_CORR );

IS_DISPLAY ( ON );

1* Extract ratio information to determine camera response */

IS_SUMMATION ( NUMERATOR, \&image );

rawnum $=($ float $)$ image $/($ (float) subheight* (float) subwidth);

IS_SUMMATION ( DENOMINATOR, \&image);

rawden=(float) image $/($ (float) subheight* (float) subwidth);

IS_SUMMATION ( NUMERATOR_CORR, \&image );

f num $=($ float $)$ image $/($ ( float $)$ subheight* (float) subwidth);

IS_SUMMATION ( DENOMINATOR_CORR, \&image );

fden $=($ float $)$ image $/($ (float) subheight* (float) subwidth);

if $(f \operatorname{den}==0)$ fden=1.0;

fratio $=$ fnum/fden;

fTemperature $=T_{\text {_high }}-\left(T_{-}\right.$high $-T_{-}$low $) *($ fratio-r_low $) /\left(r_{-}\right.$high-r_low $)$;

window=wxopen $(15,0,23,45$, NULL , ACTIVE+CENTER , 2, 65);

empty (sentence, 70);

sprintf (sentence," POINT CALIBRATION DATA:");

atsay $(1,0$, sentence);

empty (sentence, 70);

sprintf (sentence, "Ratio $=\% 5.4 \mathrm{f}$ Temperature $=\% 4.1 \mathrm{f} \% \mathrm{~s}$,

fratio, fTemperature, TUNIT );

atsay $(2,0$, sentence $)$;

empty (sentence, 70);

sprintf (sentence," Raw num = \%4.1f Raw den $=\% 4.1 \mathrm{f} "$, rawnum, rawden);

atsay $(3,0$, sentence);

empty (sentence, 70 );

sprintf (sentence," Corr num $=\% 4.1 \mathrm{f}$ Corr den $=\% 4.1 \mathrm{f} "$, fnum, fden );

atsay $(4,0$, sentence);

empty (sentence, 70);

sprintf(sentence," TEMPERATURE ESTIMATE BASED ON:" );

atsay $(6,0$, sentence);

empty (sentence, 70);

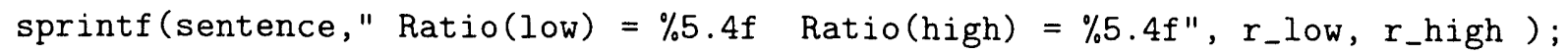

atsay $(7,0$, sentence);

empty (sentence, 70);

sprintf(sentence," Temp(low) $=\% 4.1 \mathrm{f} \% \mathrm{~s}$ Temp(high) $=\% 4.1 \mathrm{f} \% \mathrm{~s} "$, 


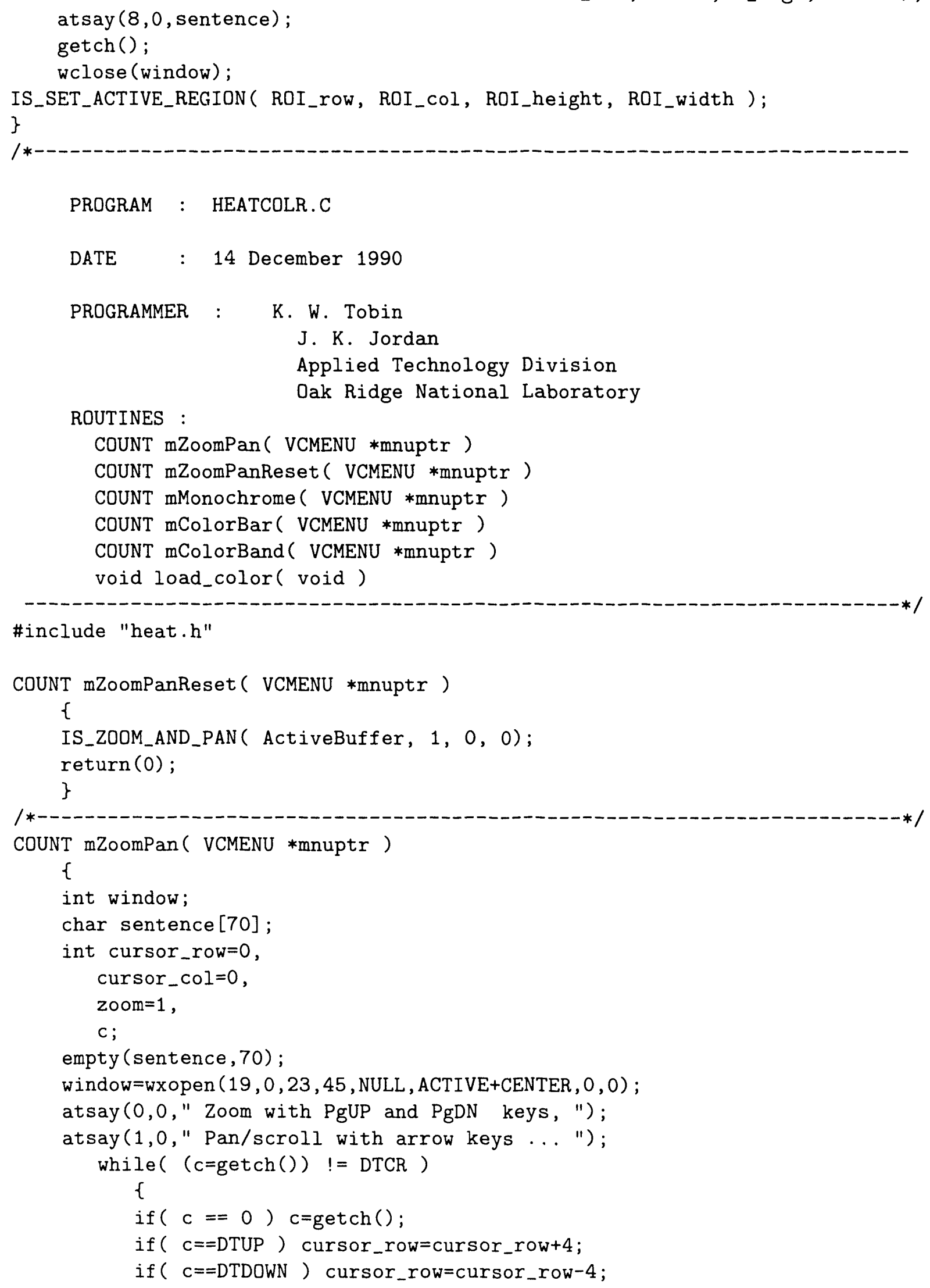




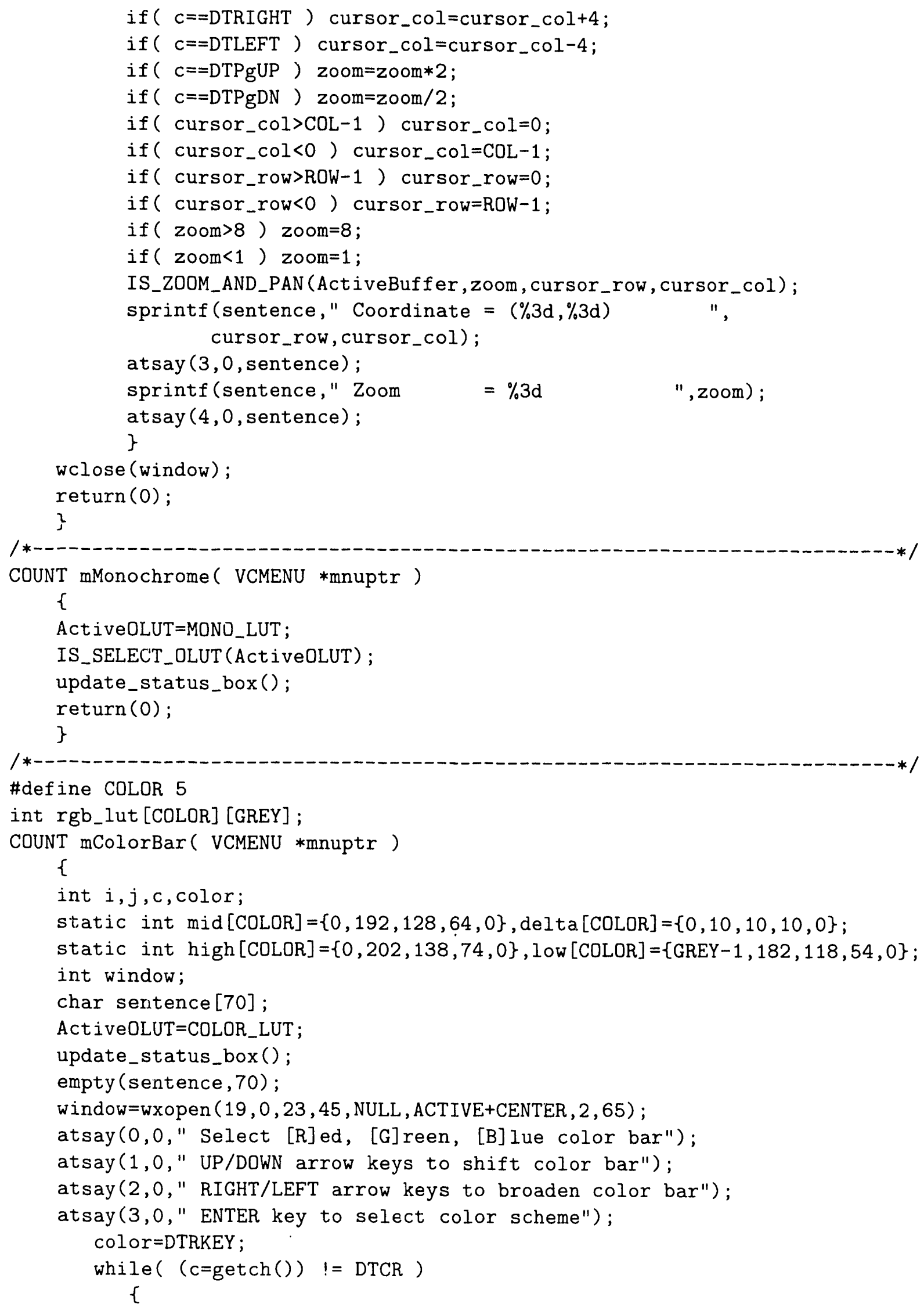




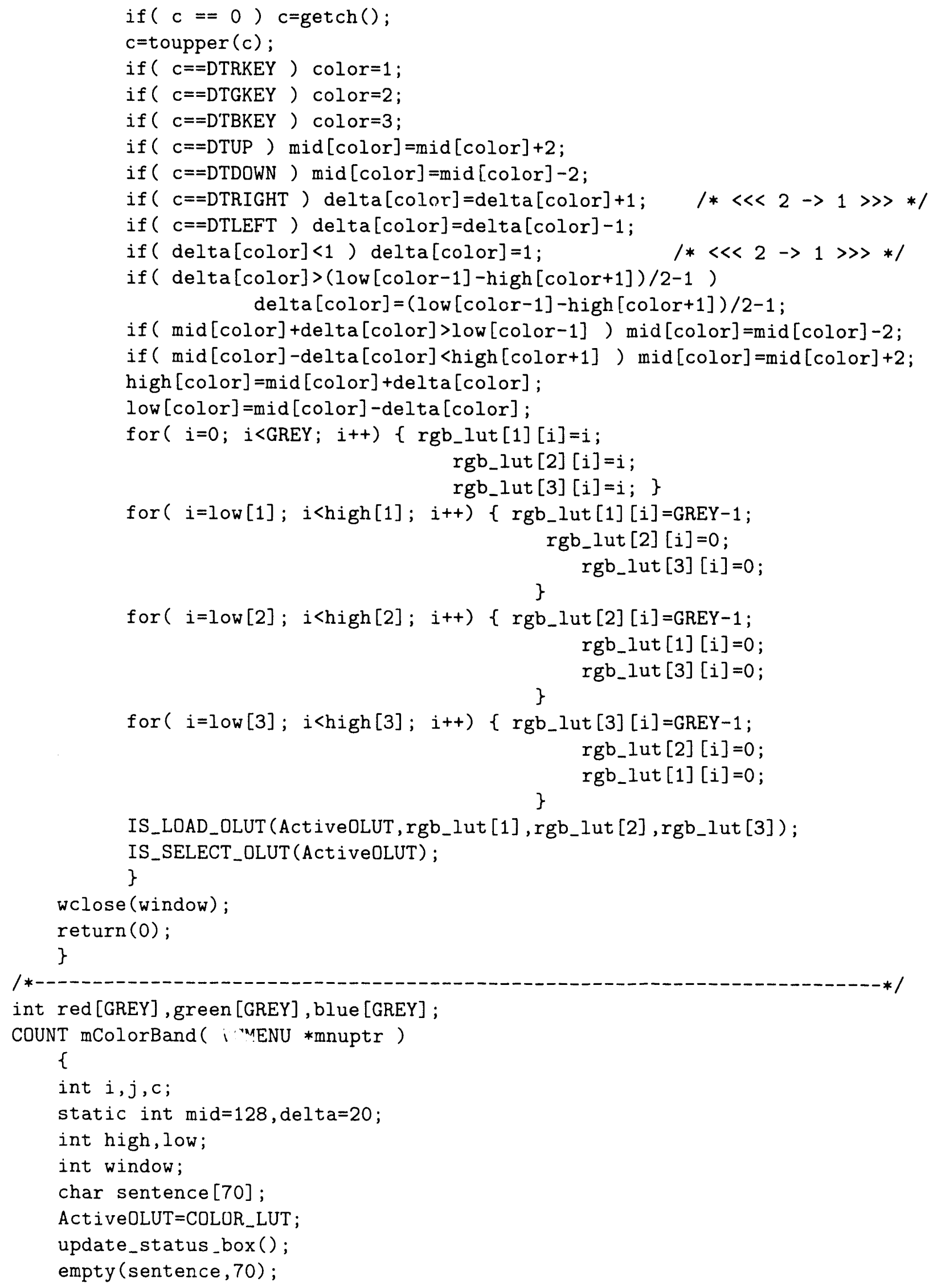




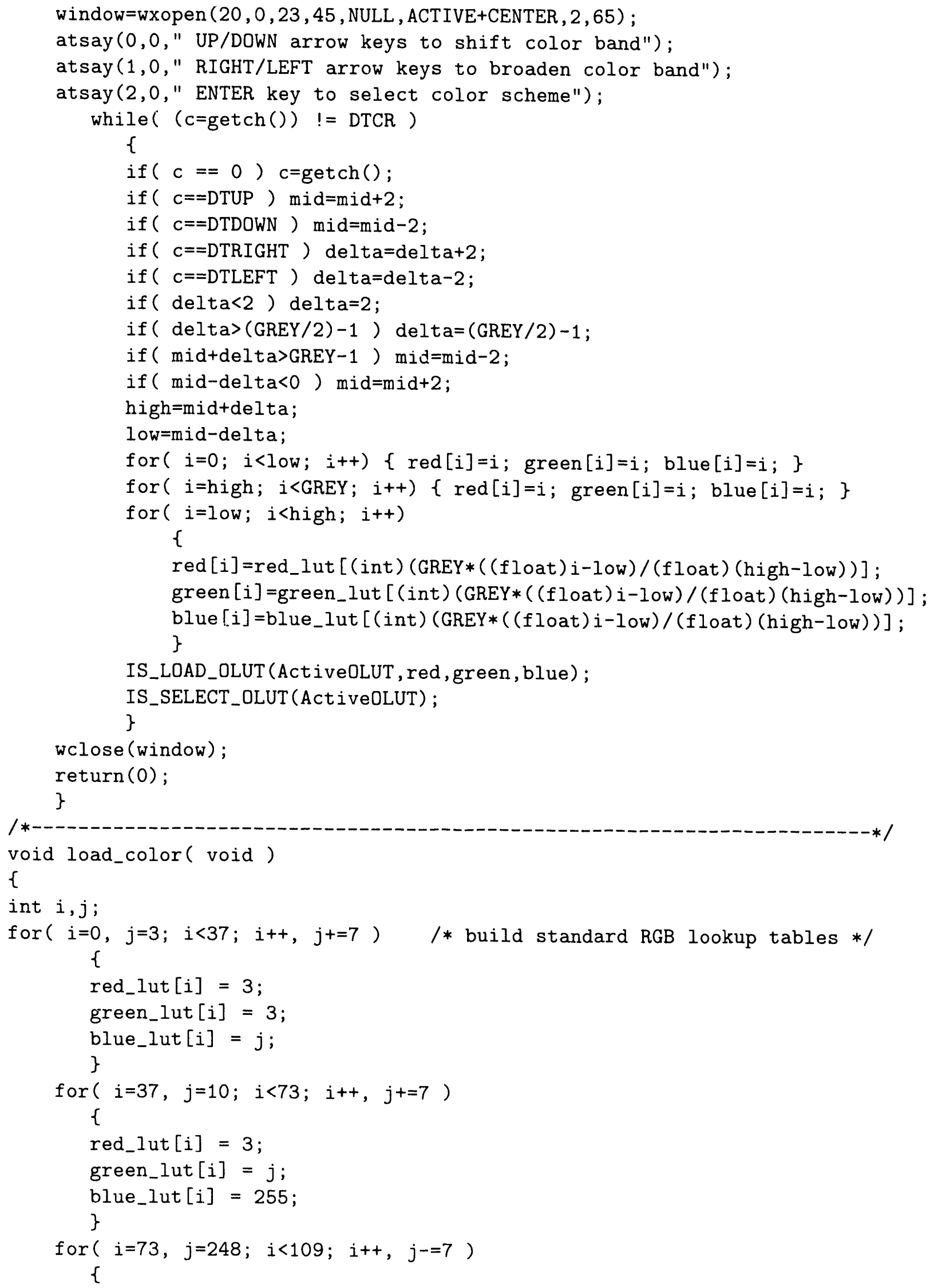




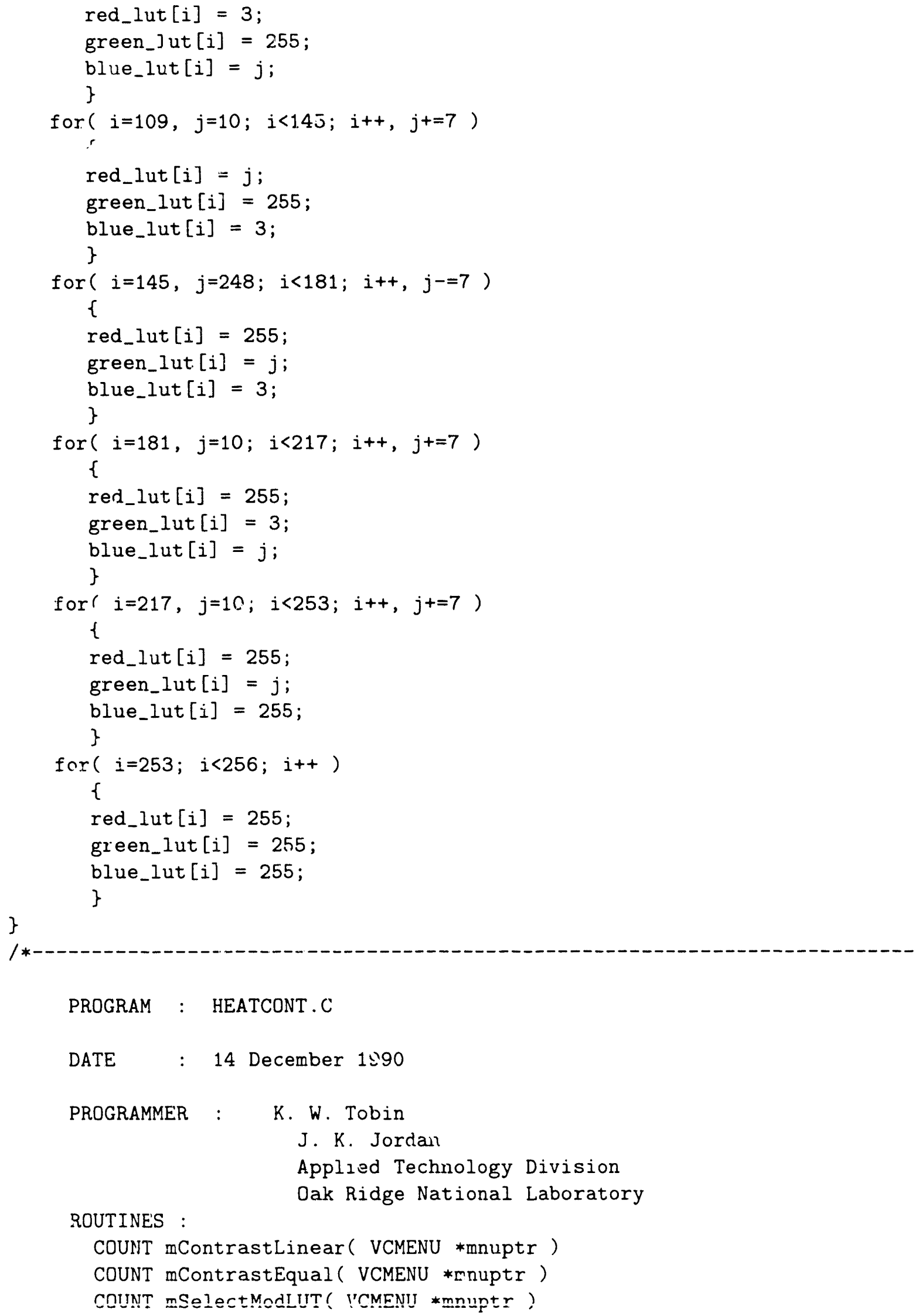




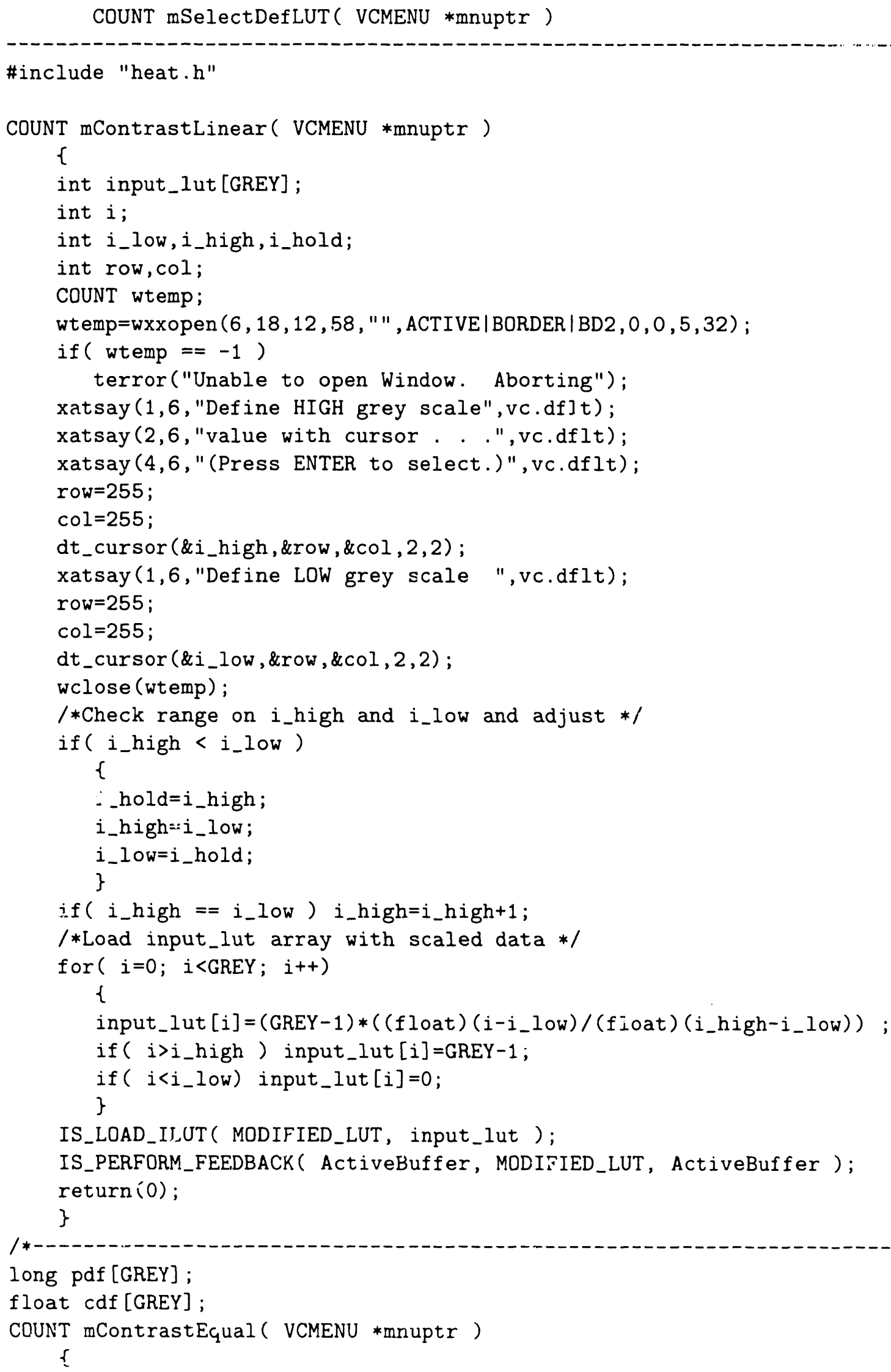




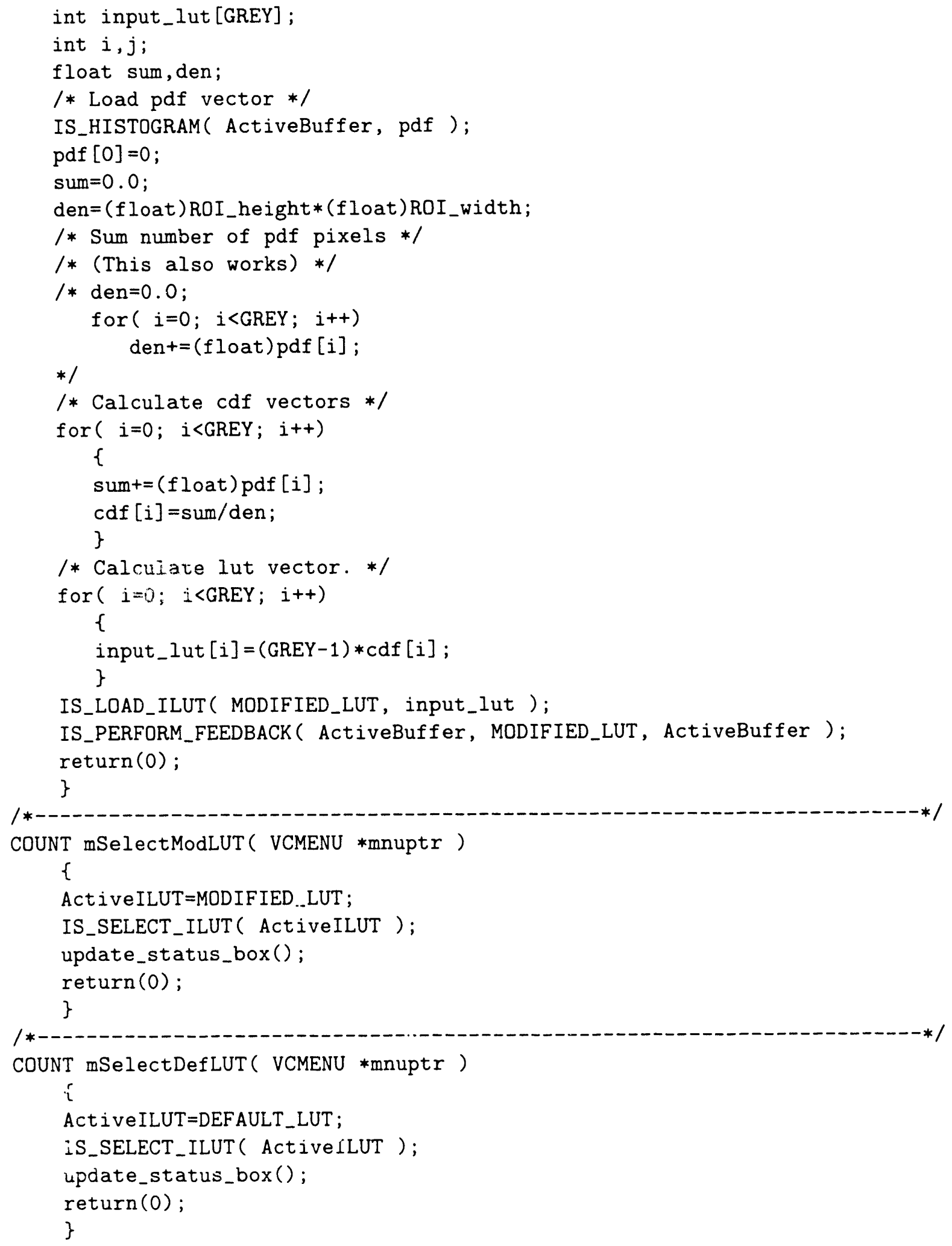

EXAMPLE RESULT FILE

HEAT-ELUX GAUGE - Ver. 1.0 (ORNI/ATD) 


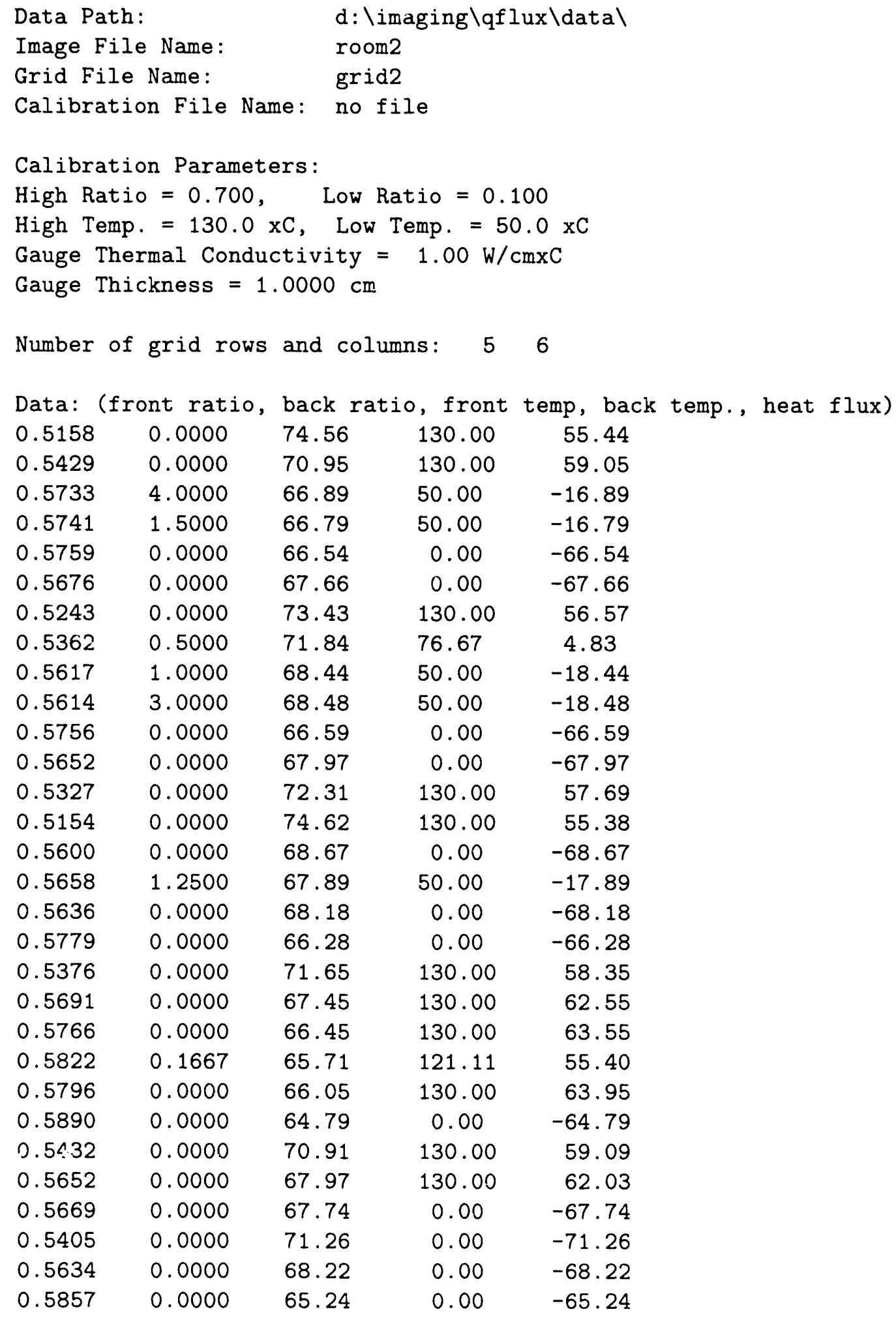




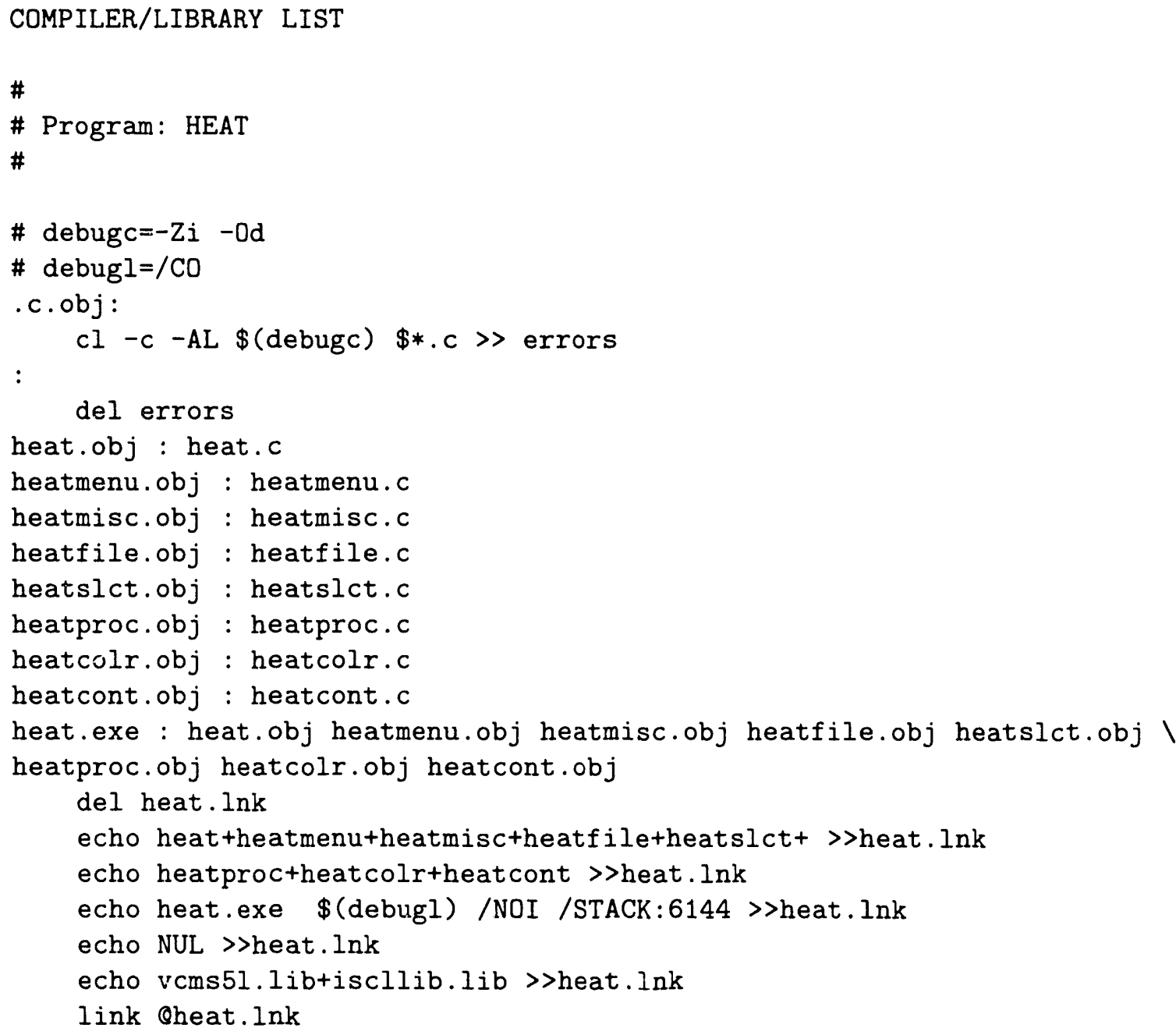



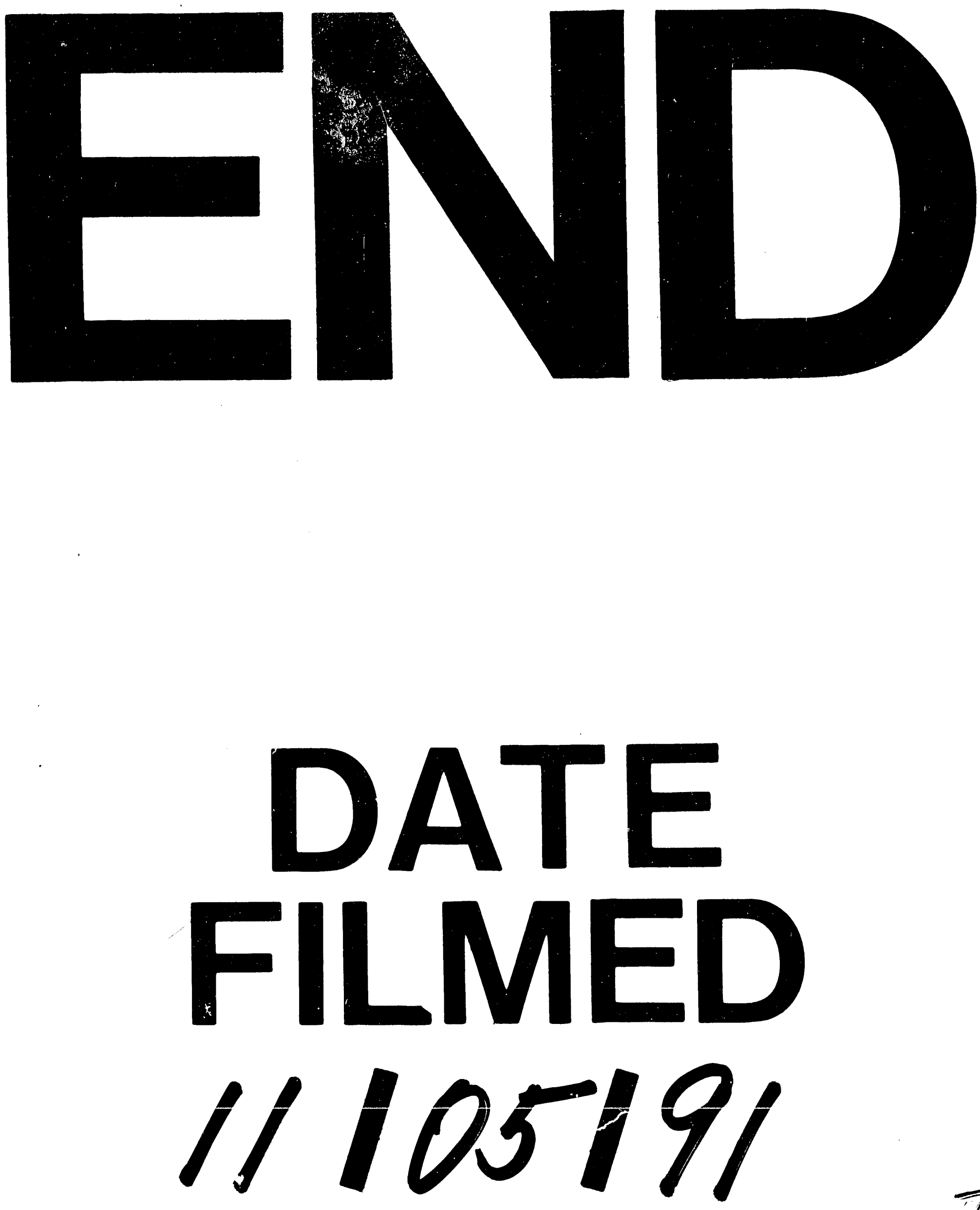
